\title{
Correlations for superpositions and decimations of Laguerre and Jacobi orthogonal matrix ensembles with a parameter
}

\author{
Peter J. Forrester* and Eric M. Rains ${ }^{\dagger}$ \\ *Department of Mathematics and Statistics, University of Melbourne, \\ Victoria 3010, Australia ; ${ }^{\dagger}$ AT\&T Research, Florham Park, NJ 07932, USA \\ (Present address: Center for Communications Research, Princeton, NJ 08540, USA)
}

A superposition of a matrix ensemble refers to the ensemble constructed from two independent copies of the original, while a decimation refers to the formation of a new ensemble by observing only every second eigenvalue. In the cases of the classical matrix ensembles with orthogonal symmetry, it is known that forming superpositions and decimations gives rise to classical matrix ensembles with unitary and symplectic symmetry. The basic identities expressing these facts can be extended to include a parameter, which in turn provides us with probability density functions which we take as the definition of special parameter dependent matrix ensembles. The parameter dependent ensembles relating to superpositions interpolate between superimposed orthogonal ensembles and a unitary ensemble, while the parameter dependent ensembles relating to decimations interpolate between an orthogonal ensemble with an even number of eigenvalues and a symplectic ensemble of half the number of eigenvalues. By the construction of new families of biorthogonal and skew orthogonal polynomials, we are able to compute the corresponding correlation functions, both in the finite system and in various scaled limits. Specializing back to the cases of orthogonal and symplectic symmetry, we find that our results imply different functional forms to those known previously.

\section{Introduction}

Dyson [9] introduced three ensembles of random unitary matrices - the circular orthogonal ensemble (COE), circular unitary ensemble (CUE) and circular symplectic ensemble (CSE). The corresponding joint eigenvalue probability density functions (PDFs) were calculated to be

$$
\frac{1}{C} \prod_{1 \leq j<k \leq n}\left|e^{i \theta_{j}}-e^{i \theta_{k}}\right|^{\beta}
$$

where $C$ is the normalization (throughout the symbol $C$ will be used to denote some normalization) and $\beta=1$ for the COE, $\beta=2$ for the CUE and $\beta=4$ for the CSE. In the theory of the COE, a technique known as integration over alternate variables has a special place. This technique draws one naturally to study statistical properties of every second eigenvalue (parity respecting correlations) as well as the statistical properties of the complete COE sequence (parity blind correlations). Thus for matrices from the ensemble $\mathrm{COE}_{2 n}(2 n \times 2 n$ members of the COE $)$ Mehta and Dyson [32] considered the statistical properties of every second eigenvalue (referred to as an alternating sequence) by integrating out the complementary alternating sequence. With the resulting distribution denoted alt $\left(\mathrm{COE}_{2 n}\right)$ they showed

$$
\operatorname{alt}\left(\mathrm{COE}_{2 n}\right)=\mathrm{CSE}_{\mathrm{n}}
$$

where on the RHS we mean the joint eigenvalue distribution of the ensemble $\mathrm{CSE}_{n}$ (below we will state similar equations with this convention without further comment). 
Prior to the work of Mehta and Dyson, Dyson [10] was led to conjecture that superimposing two independent eigenvalue sequences from the $\mathrm{COE}$ and integrating out every second eigenvalue leaves an eigenvalue sequence with the same joint distribution as the CUE, and thus

$$
\operatorname{alt}\left(\mathrm{COE}_{n} \cup \mathrm{COE}_{n}\right)=\mathrm{CUE}_{n} .
$$

This was subsequently proved by Gunson [24]. The results (1.2) and (1.3) together imply that the physically important gap probability, that is the probability that the interval $[-s, s]$ is free of eigenvalues, is inter-related for the three ensembles COE, CUE and CSE. As formulas for this quantity were known for the $\mathrm{COE}$ and CUE in terms of the Fredholm determinant of the integral operator on $[-s, s]$ with kernel $\sin \pi(x-y) / \pi(x-y)$, the inter-relationships imply a formula for the gap probability in the CSE in terms of the same Fredholm determinant 32].

Some years after the pioneering work of Dyson and Mehta, Baik and Rains 顿 were led to study the distribution of every second row of random Young tableaux specified according to some specific probability measures. In particular, a probability measure was identified for which the distribution of the even numbered rows is independent of a parameter $\alpha$ occurring in the measure. In the continuum limit, in which the integer valued row lengths go over to continuous valued variables, the PDF of the $2 n$ row lengths is specified by

$$
\frac{1}{C} \prod_{j=1}^{2 n} e^{-x_{j} / 2} \prod_{j=1}^{n} e^{A\left(x_{2 j-1}-x_{2 j}\right) / 2} \prod_{1 \leq j<k \leq 2 n}\left(x_{j}-x_{k}\right)
$$

where

$$
x_{1}>x_{2}>\cdots>x_{2 n} \geq 0,
$$

$C$ is the normalization and $A$ is the analogue of the parameter $\alpha$. For (1.4) to be normalizable we must have $A<1$. In the case $A=0$ this coincides with the joint distribution of the eigenvalues in the matrix ensemble LOE - the Laguerre orthogonal ensemble with parameter $a=0$ (for general parameter $a$ the Laguerre weight is $x^{a} e^{-x / 2}$ ). We recall the LOE distribution of $2 n$ variables with parameter $a=0$ is realized by the eigenvalues of Wishart matrices $X^{T} X$ where the real matrix $X$ has dimension $(2 n+1) \times 2 n$ and independent elements, identically distributed with the standard normal distribution $\mathrm{N}[0,1]$. Let us denote the ensemble corresponding to (1.4) by $\mathrm{LOE}_{2 n}^{A}$. With ME denoting a general matrix ensemble and the operation even(ME) denoting the distribution of the even labelled coordinates with the ordering (1.5), and thus the operation of integrating out the odd labelled coordinates, the result of Baik and Rains gives

$$
\operatorname{even}\left(\mathrm{LOE}_{2 n}^{A}\right)=\mathrm{LSE}_{n}
$$

where the PDF for the $\mathrm{LSE}_{n}$ is the $A \rightarrow-\infty$ limit of the PDF (1.4), and is thus given by

$$
\frac{1}{C} \prod_{j=1}^{n} e^{-x_{j}} \prod_{1 \leq j<k \leq n}\left(x_{j}-x_{k}\right)^{4},
$$

after re-labelling coordinates $\left\{x_{2 j-1}, x_{2 j}\right\} \mapsto x_{j}(j=1, \ldots, n)$ (see (3.16)). As a matrix ensemble the LSE refers to the Laguerre symplectic ensemble with parameter $a=0$. It can be realized as a $2 n \times 2 n$ antisymmetric matrix, in which the elements are pure imaginary numbers with each $2 \times 2$ block having a real quaternion structure. Such matrices are equivalent to block matrices of the form

$$
\left[\begin{array}{cc}
0_{n \times n} & X_{n \times n} \\
X_{n \times n}^{\dagger} & 0_{n \times n}
\end{array}\right]
$$


where $X$ is an antisymmetric complex matrix. As is evident from the transition from (1.4) to (1.7), the corresponding eigenvalue spectrum is doubly degenerate.

Implicit in [4] is the matrix ensemble corresponding to the eigenvalue PDF

$$
\frac{1}{C} \prod_{j=1}^{2 n} e^{-x_{j} / 2} \prod_{j=1}^{n} e^{A\left(x_{2 j-1}-x_{2 j}\right) / 2} \prod_{1 \leq j<k \leq n}\left(x_{2 j-1}-x_{2 k-1}\right)\left(x_{2 k}-x_{2 j}\right),
$$

where the ordering (1.5) is assumed, and as with (1.4) we must have $A<1$ for the PDF to be normalizable. When $A=0$ the matrix ensemble with this eigenvalue PDF is the superimposed ensemble

$$
\mathrm{LOE}_{n} \cup \mathrm{LOE}_{n}
$$

(two independent copies of the LOE). The $A \rightarrow-\infty$ limit of (1.8) gives the LUE, parameter $a=0$, joint eigenvalue distribution

$$
\frac{1}{C} \prod_{j=1}^{n} e^{-x_{j}} \prod_{1 \leq j<k \leq n}\left(x_{k}-x_{j}\right)^{2}
$$

where as in 1.7 ) we have re-labelled the coordinates $\left\{x_{2 j-1}, x_{2 j}\right\} \mapsto x_{j}(j=1, \ldots, n)$. As a matrix ensemble the LUE refers to the Laguerre unitary ensemble, which can be realized by matrices of the form $X^{\dagger} X$ where $X$ is a $n \times n$ matrix with independent, identically distributed complex Gaussian entries. Analogous to the derivation of (1.4), one can use arguments based on the underlying combinatorial model to show that

$$
\operatorname{even}\left(\left(\mathrm{LOE}_{n} \cup \mathrm{LOE}_{n}\right)^{A}\right)=\mathrm{LUE}_{n}
$$

independent of the parameter value $A$.

Inspired by the results (1.6) and (1.10), Forrester and Rains 18 considered general matrix ensembles with orthogonal symmetry $\mathrm{OE}_{n}(f)$ corresponding to the joint distribution

$$
\frac{1}{C} \prod_{j=1}^{n} f\left(x_{j}\right) \prod_{1 \leq j<k \leq n}\left(x_{j}-x_{k}\right) .
$$

They sought to classify all differentiable weight functions $f$ in (1.11) such that

$$
\operatorname{even}\left(\mathrm{OE}_{n}(f) \cup \mathrm{OE}_{n}(f)\right)=\mathrm{UE}_{n}(g)
$$

where $\mathrm{UE}_{n}(g)$ is the matrix ensemble with unitary symmetry corresponding to the joint distribution

$$
\frac{1}{C} \prod_{j=1}^{n} g\left(x_{j}\right) \prod_{1 \leq j<k \leq n}\left(x_{j}-x_{k}\right)^{2} .
$$

Up to linear fractional transformations only two pairs of weights $(f, g)$ were found to possess this property:

$$
(f, g)= \begin{cases}\left(e^{-x / 2}, e^{-x}\right), & x>0 \\ \left((1-x)^{(a-1) / 2},(1-x)^{a}\right), & 0<x<1 .\end{cases}
$$

The first of these is the $a=0$ Laguerre weight in (1.8), while the second is an example of the Jacobi weight $x^{b}(1-x)^{a}$ with parameter $b=0$. Furthermore, it was proved [18 that the statement (1.12) is equivalent to the statement

$$
\text { even }\left(\mathrm{OE}_{2 n}(f)\right)=\mathrm{SE}_{n}\left((g / f)^{2}\right)
$$

where $\mathrm{SE}_{n}(h)$ denotes the matrix ensemble with symplectic symmetry corresponding to the joint distribution

$$
\frac{1}{C} \prod_{j=1}^{n} h\left(x_{j}\right) \prod_{1 \leq j<k \leq n}\left(x_{j}-x_{k}\right)^{4}
$$


Our interests in this work are parameter dependent generalizations of the orthogonal ensembles, and superimposed orthogonal ensembles, specified by the weights $f$ in (1.13). For the weight $f(x)=e^{-x / 2}$, $(x>0)$, these are precisely the ensembles with PDFs (1.4) and (1.8). For the weight $f(x)=(1-x)^{(a-1) / 2}$, $0<x<1$, the parameter dependent ensembles are specified by the PDFs

$$
\frac{1}{C} \prod_{j=1}^{2 n}\left(1-x_{j}\right)^{(a-1) / 2} \prod_{l=1}^{n}\left(\frac{1-x_{2 l-1}}{1-x_{2 l}}\right)^{-A / 2} \prod_{1 \leq j<k \leq 2 n}\left(x_{j}-x_{k}\right)
$$

and

$$
\frac{1}{C} \prod_{j=1}^{2 n}\left(1-x_{j}\right)^{(a-1) / 2} \prod_{l=1}^{n}\left(\frac{1-x_{2 l-1}}{1-x_{2 l}}\right)^{-A / 2} \prod_{1 \leq j<k \leq n}\left(x_{2 j-1}-x_{2 k-1}\right)\left(x_{2 j}-x_{2 k}\right)
$$

where, for convenience, we shift the origin and scale the variable $x$ relative to that used in (1.13) so that

$$
-1<x_{j}<1 \quad(j=1, \ldots, 2 n) .
$$

For (1.16) and (1.17) to be normalizable we must have $A<a+1$. We remark that after shifting the origin back to that used in (1.13) by $x_{j} \mapsto \frac{1}{2}\left(x_{j}+1\right)$, then scaling the variables and parameters

$$
x_{j} \mapsto x_{j} / L, \quad a \mapsto L, \quad A \mapsto L A
$$

and taking the limit $L \rightarrow \infty,(1.16)$ and (1.17) reduce to (1.4) and (1.7) respectively.

We note that with respect to each even labelled coordinate the parameter $A$ in (1.16) and (1.17) can be viewed as a change of parameter $a \mapsto a+A$ in the Jacobi weight. Let us make this change in (1.13) and form the identities (1.12) and (1.14). If we then divide both sides of the resulting identities by $\prod_{l=1}^{2 n}\left(1-x_{l}\right)^{A / 2}$ so that in each case the RHS is independent of $A$, we deduce that

$$
\begin{gathered}
\text { even }\left(\mathrm{OE}_{n}\left(f_{\mathrm{o}}, f_{\mathrm{e}}\right) \cup \mathrm{OE}_{n}\left(f_{\mathrm{o}}, f_{\mathrm{e}}\right)\right)=\mathrm{UE}_{n}(g), \\
\quad \operatorname{even}\left(\mathrm{OE}_{2 n}\left(f_{\mathrm{o}}, f_{\mathrm{e}}\right)\right)=\operatorname{SE}_{n}\left(\left(g /\left.f_{\mathrm{e}}\right|_{A=0}\right)^{2}\right),
\end{gathered}
$$

with

$$
\left(f_{\mathrm{o}}, f_{\mathrm{e}}, g\right)=\left((1-x)^{(a-A-1) / 2},(1-x)^{(a+A-1) / 2},(1-x)^{a}\right), \quad-1<x<1 .
$$

In (1.18), assuming the ordering $x_{1}>x_{2}>\cdots>x_{2 n}, \mathrm{OE}_{n}\left(f_{\mathrm{o}}, f_{\mathrm{e}}\right) \cup \mathrm{OE}_{n}\left(f_{\mathrm{o}}, f_{\mathrm{e}}\right)$ refers to the ensemble with PDF

$$
\prod_{j=1}^{n} f_{\mathrm{o}}\left(x_{2 j-1}\right) f_{\mathrm{e}}\left(x_{2 j}\right) \prod_{1 \leq j<k \leq n}\left(x_{2 j}-x_{2 k}\right)\left(x_{2 j-1}-x_{2 k-1}\right),
$$

while in (1.19) $\mathrm{OE}_{2 n}\left(f_{\mathrm{o}}, f_{\mathrm{e}}\right)$ refers to the ensemble with PDF

$$
\prod_{j=1}^{n} f_{\mathrm{o}}\left(x_{2 j-1}\right) f_{\mathrm{e}}\left(x_{2 j}\right) \prod_{1 \leq j<k \leq 2 n}\left(x_{j}-x_{k}\right)
$$

An analogous argument starting with the Laguerre case of (1.13), modified so that $x$ therein is replaced by $(1+A) x$, can be used to deduce (1.4) and (1.10).

The parameter dependent PDFs (1.4), (1.8), (1.16) and (1.17) each have at least two interpretations in distinct applied settings. One, already made explicit [4, 2] in the case of (1.4), is as the continuum limit of certain measures on partitions. These measures in turn are intimately related to increasing subsequence problems [3, 8, 6], growth models 27, 28] and non-intersecting lattice paths [30, 25, 15, 26. The other is as the eigenvalue PDF for certain computable ensembles of random matrices. Development 
of these settings will be undertaken in a separate publication [19]. Here we seek the evaluation of the multi-point correlation functions, both parity aware and parity blind (recall the terminology from the first paragraph) associated with the probability densities (1.4), (1.8), (1.16) and (1.17). Let us first revise the definition of a multi-point correlation function. Consider a general PDF

$$
p\left(x_{1}, \ldots, x_{n} ; y_{1}, \ldots, y_{n}\right)
$$

which is symmetric in $\left\{x_{j}\right\}_{j=1, \ldots, n}$ and $\left\{y_{j}\right\}_{j=1, \ldots, n}$ with the support of $p$ on some region $I$ of the real line for each coordinate. This can be thought of as the PDF for a two species system of particles free to move on $I$. The $\left(k_{1}, k_{2}\right)$-point correlation function for $k_{1}$ particles of species $x$ and $k_{2}$ particles of species $y$ is defined as

$$
\begin{aligned}
& \rho_{\left(k_{1}, k_{2}\right)}\left(x_{1}, \ldots, x_{k_{1}} ; y_{1}, \ldots, y_{k_{2}}\right)=n(n-1) \cdots\left(n-k_{1}+1\right) n(n-1) \cdots\left(n-k_{2}+1\right) \\
& \times \prod_{l=k_{1}+1}^{n} \prod_{l^{\prime}=k_{2}+1}^{n} \int_{I} d x_{l} \int_{I} d y_{l^{\prime}} p\left(x_{1}, \ldots, x_{n} ; y_{1}, \ldots, y_{n}\right) .
\end{aligned}
$$

All the PDFs (1.4), (1.8), (1.16) and (1.17) can be written in the form (1.23) with $x_{1}, \ldots, x_{n}$ denoting the odd labelled particles in the ordering (1.5), and $y_{1}, \ldots, y_{n}$ denoting the even labelled particles in the ordering (1.5).

In the parity aware cases, it follows from general formulas due to Rains 36 that for the PDFs (1.8) and (1.16) the $\left(k_{1}, k_{2}\right)$-point correlation functions have the determinant structure

$$
\begin{aligned}
& \rho_{\left(k_{1}, k_{2}\right)}\left(x_{1}, \ldots, x_{k_{1}} ; y_{1}, \ldots, y_{k_{2}}\right) \\
& =\operatorname{det}\left[\begin{array}{cc}
{\left[K_{\mathrm{oo}}\left(x_{j}, x_{l}\right)\right]_{j, l=1, \ldots, k_{1}}} & {\left[K_{\mathrm{oe}}\left(x_{j}, y_{l}\right)\right]_{\substack{j=1, \ldots, k_{1} \\
l=1, \ldots, k_{2}}}} \\
{\left[K_{\mathrm{eo}}\left(y_{j}, x_{l}\right)\right]_{\substack{j=1, \ldots, k_{2} \\
l=1, \ldots, k_{1}}}} & {\left[K_{\mathrm{ee}}\left(y_{j}, y_{l}\right)\right]_{j, l=1, \ldots, k_{2}}}
\end{array}\right]
\end{aligned}
$$

for certain functions $K_{\mathrm{oo}}, K_{\mathrm{oe}}, K_{\mathrm{eo}}$ and $K_{\mathrm{ee}}$. The latter are defined in terms of arbitrary polynomials $p_{j}(y), Q_{j}(x)$ of degree $j$, as well as the inverse of the matrix

$$
\left[\int_{0}^{\infty} d y e^{-(1+A) y / 2} p_{j}(y) \int_{y}^{\infty} d x e^{-(1-A) x / 2} Q_{k}(x)\right]_{j, k=0, \ldots, n-1}
$$

in the case of (1.4), and the inverse of the matrix

$$
\left[\int_{-1}^{1} d y(1-y)^{(a-A-1) / 2} p_{j}(y) \int_{y}^{1} d x(1-x)^{(a+A-1) / 2} Q_{k}(x)\right]_{j, k=0, \ldots, n-1}
$$

in the case of (1.16). Also, a general formula of [36] gives that for the PDFs (1.4) and (1.17) the $\left(k_{1}, k_{2}\right)$ point correlation functions have the quaternion determinant (the definition of a quaternion determinant is revised in Section 3i) structure

$$
\begin{aligned}
& \rho_{\left(k_{1}, k_{2}\right)}\left(x_{1}, \ldots, x_{k_{1}} ; y_{1}, \ldots, y_{k_{2}}\right) \\
& \operatorname{qdet}\left[\begin{array}{cc}
{\left[f_{\mathrm{oo}}\left(x_{j}, x_{l}\right)\right]_{j, l=1, \ldots, k_{1}}} & {\left[f_{\mathrm{oe}}\left(x_{j}, y_{l}\right)\right]_{\substack{j=1, \ldots, k_{1} \\
l=1, \ldots, k_{2}}}} \\
{\left[f_{\mathrm{eo}}\left(y_{j}, x_{l}\right)\right]_{\substack{j=1, \ldots, k_{2} \\
l=1, \ldots, k_{1}}}} & {\left[f_{\mathrm{ee}}\left(y_{j}, y_{l}\right)\right]_{j, l=1, \ldots, k_{2}}}
\end{array}\right]
\end{aligned}
$$

where the $f_{s_{1} s_{2}}$ are $2 \times 2$ matrices with elements defined in terms of arbitrary $j$ th degree polynomials $R_{j}(x)$, together with quantities which differ in their specification depending on whether one is considering (1.4) or (1.17). For (1.4) these quantities are

$$
\Phi_{j}^{\mathrm{e}}(x):=\int_{x}^{\infty} e^{-t / 2} e^{A(t-x) / 2} R_{j}(t) d t
$$


and the inverse of the antisymmetric matrix

$$
\left[\int_{0}^{\infty} e^{-x / 2} R_{j}(x) \Phi_{k}^{\mathrm{e}}(x) d x\right]_{j, k=0, \ldots, 2 n-1}
$$

while for (1.17) they are

$$
\Phi_{j}^{\mathrm{e}}(x):=\int_{x}^{1}(1-t)^{(a-1) / 2}\left(\frac{1-t}{1-x}\right)^{-A / 2} R_{j}(t) d t
$$

and the inverse of the antisymmetric matrix

$$
\left[\int_{-1}^{1}(1-x)^{(a-1) / 2} R_{j}(x) \Phi_{k}^{\mathrm{e}}(x) d x\right]_{j, k=0, \ldots, 2 n-1} .
$$

Regarding the parity blind correlations, $\rho_{k}\left(x_{1}, \ldots, x_{k}\right.$ ) (here the symbol $x_{j}$ is used in its original sense of (1.4), 1.8), (1.16) and (1.17) ) we note that because (1.8) and (1.17) do not vanish when $x_{2 j-1}=x_{2 j}$, $\rho_{k}$ does not vanish at coincident points and so cannot have a simple determinental form analogous to (1.16). However (1.4) and (1.16) can both be written in a form involving a Pfaffian factor,

$$
\prod_{j=1}^{2 n} w\left(x_{j}\right) \prod_{1 \leq j<l \leq 2 n}\left(x_{j}-x_{l}\right) \operatorname{Pf}\left[\epsilon\left(x_{j}, x_{l}\right]_{j, l=1, \ldots, 2 n},\right.
$$

for which the general structure of the $k$-point correlation is known [31] in terms of a quaternion determinant

$$
\rho_{k}\left(x_{1}, \ldots, x_{k}\right)=\operatorname{qdet}\left[f\left(x_{j}, x_{l}\right)\right]_{j, l=1, \ldots, k}
$$

where $f$ is a $2 \times 2$ matrix defined in terms of the same quantities as those specifying (1.27).

We find that in the case of the parameter dependent Laguerre ensembles (1.4) and (1.8), the correlations can be written in terms of

$$
K_{n}^{L}(x, y):=e^{-(x+y) / 2} \sum_{l=0}^{n-1} \frac{1}{h_{l}^{L}} L_{l}(x) L_{l}(y),
$$

where $L_{l}(x)$ denotes the Laguerre polynomial of degree $l$ and parameter $a=0$, with the orthogonality property

$$
\int_{0}^{\infty} e^{-t} L_{j}(t) L_{k}(t) d t=h_{j}^{L} \delta_{j, k}, \quad h_{j}^{L}=1 .
$$

The function $K_{n}^{L}$ is familiar as determining the correlation function of the Laguerre unitary ensemble with parameter $a=0$. The latter has the eigenvalue PDF (1.9). Explicitly, the $k$-point correlation function is given in terms of (1.32) by

$$
\rho_{k}\left(x_{1}, \ldots, x_{k}\right)=\operatorname{det}\left[K_{n}^{L}\left(x_{j}, x_{l}\right)\right]_{j, l=1, \ldots, k} .
$$

Similarly, in the case of the parameter dependent Jacobi ensembles (1.16) and 1.17), the correlations can be written in terms of

$$
K_{n}^{J}(x, y):=(1-x)^{(a-1) / 2}(1-y)^{(a-1) / 2} \sum_{l=0}^{n-1} \frac{1}{h_{l}^{J}} P_{l}^{(a, 0)}(x) P_{l}^{(a, 0)}(y)
$$

where $P_{l}^{(a, 0)}(x)$ denotes the Jacobi polynomial of degree $l$ and parameter $b=0$, with the orthogonality property

$$
\int_{-1}^{1}(1-t)^{a} P_{j}^{(a, 0)}(t) P_{k}^{(a, 0)}(t) d t=h_{j}^{J} \delta_{j, k}, \quad h_{j}^{J}=\frac{2^{a+1}}{2 j+a+1} .
$$


We remark that

$$
\rho_{k}\left(x_{1}, \ldots, x_{k}\right)=\operatorname{det}\left[\left(1-x_{l}\right) K_{n}^{J}\left(x_{j}, x_{l}\right)\right]_{j, l=1, \ldots, k}
$$

is the $k$-point correlation function for the eigenvalue $\mathrm{PDF}$

$$
\frac{1}{C} \prod_{l=1}^{n}\left(1-x_{l}\right)^{a} \prod_{1 \leq j<k \leq n}\left(x_{k}-x_{j}\right)^{2}, \quad\left|x_{l}\right|<1,
$$

which corresponds to the Jacobi unitary ensemble with parameter $b=0$.

Let us now turn to the plan of the paper. In Section 2 we take up the problem of the explicit computation of the entries of (1.16), and in Section 3 we compute the explicit form of the entries in (1.27) and (1.31). Various scaled limits of the correlations are computed in Section 4, while we finish in Section 5 by relating the correlation functions found in this study to correlation and distribution functions known from previous studies.

\section{Correlations for superimposed orthogonal ensembles with a parameter}

Consider a PDF of the form

$$
\prod_{j=1}^{n} w_{\mathrm{o}}\left(x_{j}\right) w_{\mathrm{e}}\left(y_{j}\right) \prod_{1 \leq j<k \leq n}\left(x_{j}-x_{k}\right)\left(y_{j}-y_{k}\right) \operatorname{det}\left[\kappa\left(x_{j}, y_{k}\right)\right]_{j, k=1, \ldots, n},
$$

where $x_{j}, y_{j} \in \mathbb{R}$ (although the support of $w_{\mathrm{o}}, w_{\mathrm{e}}$ may be some subset of $\mathbb{R}$ ). A general formula of [36] gives that the $\left(k_{1}, k_{2}\right)$-point correlation function is given by (1.24) with

$$
\begin{aligned}
K_{\mathrm{oo}}\left(x, x^{\prime}\right) & =\sum_{j, k=0}^{n-1} w_{\mathrm{o}}(x) Q_{j}(x) M_{j k}^{-t} \int_{-\infty}^{\infty} \kappa\left(x^{\prime}, u\right) w_{\mathrm{e}}(u) p_{k}(u) d u \\
K_{\mathrm{oe}}(x, y) & =\sum_{j, k=0}^{n-1} w_{\mathrm{o}}(x) Q_{j}(x) M_{j k}^{-t} w_{\mathrm{e}}(y) p_{k}(y) \\
K_{\mathrm{eo}}(y, x) & =-\kappa(x, y)+\sum_{j, k=0}^{n-1}\left(\int_{-\infty}^{\infty} \kappa(u, y) w_{\mathrm{e}}(u) Q_{j}(u) d u\right) M_{j k}^{-t}\left(\int_{-\infty}^{\infty} \kappa(x, v) w_{\mathrm{o}}(v) p_{k}(v)\right) d v \\
K_{\mathrm{ee}}\left(y, y^{\prime}\right) & =\sum_{j, k=0}^{n-1}\left(\int_{-\infty}^{\infty} \kappa(v, y) w_{\mathrm{o}}(v) Q_{j}(v) d v\right) M_{j k}^{-t} w_{\mathrm{e}}\left(y^{\prime}\right) p_{k}\left(y^{\prime}\right)
\end{aligned}
$$

where ${ }^{-t}$ denotes the operation of taking the transpose of the inverse, $p_{j}(y)$ and $Q_{j}(x)$ are as in (1.25) and $\left[M_{j k}\right]$ is the matrix with entries

$$
M_{j k}=\int_{-\infty}^{\infty} d x w_{\mathrm{o}}(x) Q_{j}(x) \int_{-\infty}^{\infty} d y \kappa(x, y) w_{\mathrm{e}}(y) p_{k}(y) .
$$

This result is relevant to (1.8) and (1.16) because both these PDFs can be written in the form (2.1) with

$$
\kappa(x, y)= \begin{cases}e^{A(x-y) / 2} \chi_{x>y}, & \text { Laguerre case } \\ \left(\frac{1-x}{1-y}\right)^{-A / 2} \chi_{x>y}, & \text { Jacobi case }\end{cases}
$$

where $\chi_{T}=1$ if $T$ is true and 0 otherwise, a fact which can be seen by making note of the following determinant identity. 
Lemma 1. For the orderings

$$
x_{1}>\cdots>x_{n}, \quad y_{1}>\cdots>y_{n}
$$

we have

$$
\operatorname{det}\left[\chi_{x_{j}-y_{k}>0}\right]_{j, k=1, \ldots, n}=\chi_{x_{1}>y_{1}>\cdots>x_{n}>y_{n}} .
$$

Proof. For the ordering $x_{1}>y_{1}>\cdots>x_{n}>y_{n}$ the determinant is triangular with 1's down the diagonal, so (2.6) is correct in this case. All other orderings must have at least two $x$ 's (or two $y$ 's) in succession. The corresponding rows (or columns) in the determinant will then be equal so the determinant vanishes.

Thus with $\kappa(x, y)$ given by (2.4), we can substitute (2.6) times

$$
\prod_{j=1}^{n} e^{A\left(x_{j}-y_{j}\right) / 2} \quad \text { Laguerre case, } \quad \prod_{j=1}^{n}\left(\frac{1-x_{j}}{1-y_{j}}\right)^{-A / 2} \quad \text { Jacobi case }
$$

for the determinant in (2.1), and

$$
w_{\mathrm{o}}(x)=w_{\mathrm{e}}(x)= \begin{cases}e^{-x / 2}(x>0), & \text { Laguerre case, } \\ (1-x)^{(a-1) / 2}(-1<x<1), & \text { Jacobi case }\end{cases}
$$

provided the ordering (2.5) is assumed. On this latter point, (2.1) is a symmetric function of the $x$ 's and $y$ 's (separately), so the ordering constraint (2.5) is in fact irrelevant.

For general PDFs (2.1) there is of course no explicit formula available for the inverse of $\left[M_{j k}\right]$ which is required in (2.2). However, for the particular PDFs (1.8) and (1.16), and thus $\kappa(x, y)$ given by (2.4), this problem can be overcome by choosing $\left\{p_{j}(y)\right\}$ and $\left\{Q_{k}(x)\right\}$ to have the biorthogonality property

$$
\int_{-\infty}^{\infty} d y w_{\mathrm{e}}(y) p_{j}(y) \int_{y}^{\infty} d x w_{\mathrm{o}}(x) Q_{k}^{L}(x)=n_{j} \delta_{j, k} .
$$

These polynomials are given simply in terms of the orthogonal polynomials $\left\{L_{j}(x)\right\}$ in the Laguerre case and $\left\{P_{j}^{(a, 0)}(x)\right\}$ in the Jacobi case.

Proposition 2. The sets of polynomials $\left\{L_{j}(x)\right\}_{j=0,1, \ldots}$ and $\left\{Q_{j}^{L}(x)\right\}_{j=0,1, \ldots}$ where

$$
Q_{j}^{L}(x)=\frac{2}{A-1} e^{(1-A) x / 2} \frac{d}{d x}\left(e^{-(1-A) x / 2} L_{j}(x)\right)
$$

have the biorthogonality property

$$
\int_{0}^{\infty} d t e^{-(1+A) t / 2} L_{p}(t) \int_{t}^{\infty} d x e^{-(1-A) x / 2} Q_{q}^{J}(x)=\mathcal{N}_{p}^{L} \delta_{p, q}, \quad \mathcal{N}_{p}^{L}:=-\frac{2}{A-1},
$$

while the sets of polynomials $\left\{P_{j}^{(a, 0)}(x)\right\}_{j=0,1, \ldots}$ and $\left\{Q_{j}^{J}(x)\right\}_{j=0,1, \ldots}$ where

$$
Q_{j}^{J}(x)=-\frac{(1-x)^{-(a-A-1) / 2}}{j+(a-A+1) / 2} \frac{d}{d x}\left((1-x)^{(a-A+1) / 2} P_{j}^{(a, 0)}(x)\right)
$$

have the biorthogonality property

$$
\begin{aligned}
& \int_{-1}^{1} d t(1-t)^{(a+A-1) / 2} P_{p}(t) \int_{t}^{1} d x(1-x)^{(a-A-1) / 2} Q_{q}(x)=\mathcal{N}_{p}^{J} \delta_{p, q} \\
& \mathcal{N}_{p}^{J}:=\frac{2^{a+1}}{(a+1+2 p)(p+(a-A+1) / 2)} .
\end{aligned}
$$


Proof. Substituting (2.9) in the LHS of (2.10) and making use of (1.33) gives the RHS of (2.10). Thus it only remains to check that $Q_{j}^{L}(x)$ is indeed a polynomial of degree $j$. This latter point follows by inspection of (2.9). The verification of (2.12) is done analogously.

The biorthogonality properties (2.10) and (2.12) allow $M_{j k}^{-t}$ in (2.2) to be replaced by $\mathcal{N}_{j}^{-1} \delta_{j, k}$. The double sums in (2.2) then collapse to single sums. Furthermore, taking note of the values of the normalizations from (1.33) and (1.36) we see that (2.9) and (2.11) can be rewritten

$$
\begin{aligned}
\frac{Q_{j}^{L}(x)}{\mathcal{N}_{j}^{L}} & =-\frac{1}{h_{j}^{L}} e^{(1-A) x / 2} \frac{d}{d x}\left(e^{-(1-A) x / 2} L_{j}(x)\right) \\
\frac{Q_{j}^{J}(x)}{\mathcal{N}_{j}^{J}} & =-\frac{1}{h_{j}^{J}}(1-x)^{-(a-A-1) / 2} \frac{d}{d x}\left((1-x)^{(a-A+1) / 2} P_{j}^{(a, 0)}(x)\right) .
\end{aligned}
$$

It thus follows that the quantities in (2.2) can then be expressed simply in terms of the functions $K_{n}^{L}$ and $K_{n}^{J}$ introduced in (1.32) and (1.35).

Proposition 3. The $\left(k_{1}, k_{2}\right)$ point parity respecting correlation for the PDF $(1.8)$ is given by (1.24) with

$$
\begin{aligned}
K_{\mathrm{oo}}^{L}\left(x, x^{\prime}\right) & =-e^{-A\left(x-x^{\prime}\right) / 2} \frac{\partial}{\partial x}\left\{e^{A x / 2} \int_{0}^{x^{\prime}} e^{-A u / 2} K_{n}^{L}(x, u) d u\right\} \\
K_{\mathrm{oe}}^{L}(x, y) & =-e^{-A x / 2} \frac{\partial}{\partial x}\left\{e^{A x / 2} K_{n}^{L}(x, y)\right\} \\
K_{\mathrm{eo}}^{L}(y, x) & =-e^{A(x-y) / 2} \chi_{x>y}+e^{A x / 2} \int_{0}^{x} e^{-A v / 2} K_{n}^{L}(v, y) d v \\
K_{\mathrm{ee}}^{L}\left(y, y^{\prime}\right) & =K_{n}^{L}\left(y, y^{\prime}\right)
\end{aligned}
$$

(we have appended the superscripts $L$ on the LHS as notation for the Laguerre case (1.8)). Similarly, the $\left(k_{1}, k_{2}\right)$ point parity respecting correlation for the PDF 1.10$)$ - the Jacobi case to be denoted by appending a superscript $J$ - is given by (1.24) with

$$
\begin{aligned}
K_{\mathrm{oo}}^{J}\left(x, x^{\prime}\right) & =-\left(\frac{1-x}{1-x^{\prime}}\right)^{A / 2} \frac{\partial}{\partial x}\left\{(1-x)^{1-A / 2} \int_{-1}^{x^{\prime}}(1-u)^{A / 2} K_{n}^{J}(x, u) d u\right\} \\
K_{\mathrm{oe}}^{J}(x, y) & =-(1-x)^{A / 2} \frac{\partial}{\partial x}\left\{(1-x)^{1-A / 2} K_{n}^{J}(x, y)\right\} \\
K_{\mathrm{eo}}^{J}(y, x) & =-\left(\frac{1-x}{1-y}\right)^{-A / 2} \chi_{x>y}+(1-y)(1-x)^{-A / 2} \int_{-1}^{x}(1-v)^{A / 2} K_{n}^{J}(v, y) d v \\
K_{\mathrm{ee}}^{J}\left(y, y^{\prime}\right) & =(1-y) K_{n}^{J}\left(y, y^{\prime}\right) .
\end{aligned}
$$

We see from (2.15) that $K_{\mathrm{ee}}^{L}(x, y)$ coincides with $K_{n}^{L}(x, y)$ which we know from (1.34) determines the $k$-point correlation for the LUE. This property of the even-even correlations is equivalent to the statement (1.10). Similarly, the final formula in (2.16) implies that the $k$-point correlation for the eveneven correlations in the Jacobi case coincides with the $k$-point correlation for the JUE with parameter value $(a, b) \mapsto(a, 0)$ (recall (1.37). This result is equivalent to the statement (1.18). 


\section{Correlations for decimated orthogonal ensembles with a pa- rameter}

\subsection{Quaternion determinant formulas}

To calculate the parity respecting correlations for (1.4) and (1.16), one notes that analogous to (2.1) they have the general structure

$$
\prod_{j=1}^{n} w_{\mathrm{o}}\left(x_{2 j-1}\right) w_{\mathrm{e}}\left(x_{2 j}\right) \prod_{1 \leq j<k \leq 2 n}\left(x_{j}-x_{k}\right) \operatorname{det}\left[\kappa\left(x_{2 j-1}, x_{2 k}\right]_{j, k=1, \ldots, n} .\right.
$$

Explicitly, we choose $\kappa(x, y)$ as in (2.4), $w_{\mathrm{o}}(x)$ and $w_{\mathrm{e}}(x)$ as in (2.7), and make use of (2.6). The significance of this is that for general PDFs of the form (3.1), a result of Rains [36] gives that the parity respecting correlations have the form (1.27), and further specifies the elements of the matrix therein. Before stating the latter, we remark that the qdet operation in (1.27) is well defined on matrices $A$ with the self dual property

$$
A=Z_{2 n}^{-1} A^{T} Z_{2 n}, \quad Z_{2 n}:=\mathbf{1}_{n} \otimes\left[\begin{array}{cc}
0 & -1 \\
1 & 0
\end{array}\right] .
$$

This is equivalent to requiring that $A Z_{2 n}$ be antisymmetric. On the latter class of matrices the Pfaffian operation is well defined, and we have in fact that 11]

$$
\text { qdet } A=\operatorname{Pf}\left(A Z_{2 n}\right),
$$

which for our present purposes can be taken as the definition of qdet (in fact the results of [36] are written in terms of Pfaffians).

Let us introduce arbitrary polynomials $R_{j}(x)$ of degree $j$, and let us follow [36] and introduce the notation

$$
(\kappa \cdot f)(x)=\int_{-\infty}^{\infty} w(y) \kappa(x, y) f(y) d y
$$

where we have set

$$
w_{\mathrm{o}}(x)=w_{\mathrm{e}}(x)=w(x)
$$

(this can always be accomplished by changing the definition of $\kappa(x, y)$ ), as well as the $2 n \times 2 n$ antisymmetric matrix with elements

$$
M_{j k}=\int_{-\infty}^{\infty} d x w(x) \int_{-\infty}^{\infty} d y w(y)\left(R_{j}(x) R_{k}(y)-R_{k}(x) R_{j}(y)\right) \kappa(x, y)
$$

where the $R_{j}(x)$ are the arbitrary $j$ th degree polynomials introduced below (1.27). Then according to [36] the parity respecting correlations for the PDF (3.1) are given by (1.27) with the elements of the matrices therein specified by

$$
\begin{aligned}
f_{\mathrm{oo}}\left(x, x^{\prime}\right)= & {\left[\begin{array}{cc}
\sum_{j, k=0}^{2 n-1} w(x) R_{j}(x) M_{j k}^{-t}\left(\kappa \cdot R_{k}\right)\left(x^{\prime}\right) & -\sum_{j, k=0}^{2 n-1} w(x) R_{j}(x) M_{j k}^{-t} w\left(x^{\prime}\right) R_{k}\left(x^{\prime}\right) \\
\sum_{j, k=0}^{2 n-1}\left(\kappa \cdot R_{j}\right)(x) M_{j k}^{-t}\left(\kappa \cdot R_{k}\right)\left(x^{\prime}\right) & -\sum_{j, k=0}^{2 n-1}\left(\kappa \cdot R_{j}\right)(x) M_{j k}^{-t} w\left(x^{\prime}\right) R_{k}\left(x^{\prime}\right)
\end{array}\right] } \\
f_{\mathrm{oe}}(x, y)= & {\left[\begin{array}{cc}
\sum_{j, k=0}^{2 n-1} w(x) R_{j}(x) M_{j k}^{-t} w(y) R_{k}(y) & -\sum_{j, k=0}^{2 n-1} w(x) R_{j}(x) M_{j k}^{-t}\left(\kappa^{t} \cdot R_{k}\right)(y) \\
\sum_{j, k=0}^{2 n-1}\left(\kappa \cdot R_{j}\right)(x) M_{j k}^{-t} w(y) R_{k}(y) & -\kappa(x, y)-\sum_{j, k=0}^{2 n-1}\left(\kappa \cdot R_{j}\right)(x) M_{j k}^{-t}\left(\kappa^{t} \cdot R_{k}\right)(y)
\end{array}\right] } \\
f_{\mathrm{eo}}(y, x)= & {\left[\begin{array}{cc}
-\kappa(x, y)+\sum_{j, k=0}^{2 n-1}\left(\kappa^{t} \cdot R_{j}\right)(y) M_{j k}^{-t}\left(\kappa \cdot R_{k}\right)(x) & -\sum_{j, k=0}^{2 n-1}\left(\kappa^{t} \cdot R_{j}(y)\right) M_{j k}^{-t} w(x) R_{k}(x) \\
\sum_{j, k=0}^{2 n-1} w(y) R_{j}(y) M_{j k}^{-t}\left(\kappa \cdot R_{k}\right)(x) & -\sum_{j, k=0}^{2 n-1} w(y) R_{j}(y) M_{j k}^{-t} w(x) R_{k}(x)
\end{array}\right] } \\
f_{\mathrm{ee}}\left(y, y^{\prime}\right)= & {\left[\begin{array}{cc}
\sum_{j, k=0}^{2 n-1}\left(\kappa^{t} \cdot R_{j}\right)(y) M_{j k}^{-t} w\left(y^{\prime}\right) R_{k}\left(y^{\prime}\right) & -\sum_{j, k=0}^{2 n-1}\left(\kappa^{t} \cdot R_{j}\right)(y) M_{j k}^{-t}\left(\kappa^{t} \cdot R_{k}\right)\left(y^{\prime}\right) \\
\sum_{j, k=0}^{2 n-1} w(y) R_{j}(y) M_{j k}^{-t} w\left(y^{\prime}\right) R_{k}\left(y^{\prime}\right) & -\sum_{j, k=0}^{2 n-1} w(y) R_{j}(y) M_{j k}^{-t}\left(\kappa^{t} \cdot R_{k}\right)\left(y^{\prime}\right)
\end{array}\right] }
\end{aligned}
$$


Let us now consider the parity blind correlations. For this purpose we write the PDFs (1.4) and (1.16) in the form

$$
\prod_{j=1}^{2 n} w\left(x_{j}\right) \prod_{1 \leq j<k \leq 2 n}\left(x_{j}-x_{k}\right) \operatorname{Pf}\left[\epsilon\left(x_{j}, x_{k}\right)\right]_{j, k=1, \ldots, 2 n}
$$

where $w(x)$ is given by (3.5) with the substitution (2.7), and

$$
\epsilon(x, y)= \begin{cases}e^{A|x-y| / 2} \operatorname{sgn}(x-y), & \text { Laguerre case } \\ \left(\frac{1-x}{1-y}\right)^{-A \operatorname{sgn}(x-y) / 2} \operatorname{sgn}(x-y), & \text { Jacobi case }\end{cases}
$$

In (3.9) the notation $\operatorname{sgn}(x)$ denotes the sign of $x$ and is thus equal to 1 for $x>0$, to 0 for $x=0$ and to -1 for $x<0$. The equality of (3.8) with (1.4) and (1.16) follows from the identity

$$
\operatorname{Pf}\left[\left(\frac{f\left(x_{j}\right)}{f\left(x_{k}\right)}\right)^{\operatorname{sgn}\left(x_{j}-x_{k}\right)} \operatorname{sgn}\left(x_{j}-x_{k}\right)\right]_{j, k=1, \ldots, 2 n}=\prod_{j=1}^{n} \frac{f\left(x_{Q(2 j-1)}\right)}{f\left(x_{Q(2 j)}\right)} \varepsilon(Q),
$$

where

$$
x_{Q(2 j-1)}>x_{Q(2 j)}, \quad Q(2 j)>Q(2 j-1),(j=1, \ldots, n)
$$

and $\varepsilon(Q)$ denotes the signature of the permutation $Q$. The identity (3.10) can be seen to follow from the definition

$$
\operatorname{Pf} A=\sum_{P(2 l)>P(2 l-1)}^{*} \varepsilon(P) \prod_{l=1}^{n} a_{P(2 l), P(2 l-1)},
$$

valid for any $2 n \times 2 n$ antisymmetric matrix $A$, where the * denotes that only permutations which give rise to a unique product of the $a_{j k}$ 's are to be included. There are $(2 n-1) ! !$ such permutations, one of which for the Pfaffian (3.10) contributes the term on the RHS of (3.10). All other permutations give a contribution which cancels in pairs, so the Pfaffian (3.10) is in fact equal to this single term.

The quaternion determinant formula (1.31) for PDFs of the form (3.8) has been given by Frahm and Pichard [22]. In [22] the matrix elements of the $2 \times 2$ matrix $f$ are given in terms of skew orthogonal polynomials. In keeping with (3.7), we prefer to follow [36] and state the form of the matrix elements which involves arbitrary polynomials of degree $k, R_{k}(x)$, as well as

$$
\left(\epsilon \cdot R_{k}\right)(x):=\int_{-\infty}^{\infty} w(y) \epsilon(x, y) R_{k}(y) d y
$$

(c.f. (3.4) ) and the inverse of the antisymmetric matrix

$$
\left[\int_{-\infty}^{\infty} d x w(x) \int_{-\infty}^{\infty} d y w(y)\left(R_{j}(x) R_{k}(y)-R_{k}(x) R_{j}(y)\right) \epsilon(x, y)\right] .
$$

Comparing the definition (3.9) of $\epsilon(x, y)$ with the definition (2.4) of $\kappa(x, y)$ we see that the integral in (3.12) is unchanged if $\epsilon(x, y)$ is replaced by $\kappa(x, y)$, and is thus equal to $M_{j k}$ as defined by (3.6). With this understood, reading off from [36] we have

$$
f(x, y)=\left[\begin{array}{cc}
\sum_{j, k=0}^{2 n-1} w(x) R_{j}(x) M_{j k}^{-t}\left(\epsilon \cdot R_{k}\right)(y) & -\sum_{j, k=0}^{2 n-1} w(x) R_{j}(x) M_{j k}^{-t} w(y) R_{k}(y) \\
-\epsilon(x, y)+\sum_{j, k=0}^{2 n-1}\left(\epsilon \cdot R_{j}\right)(x) M_{j k}^{-t}\left(\epsilon \cdot R_{k}\right)(y) & -\sum_{j, k=0}^{2 n-1}\left(\epsilon \cdot R_{j}\right)(x) M_{j k}^{-t} w(y) R_{k}(y)
\end{array}\right] .
$$




\subsection{Skew orthogonal polynomials}

Our ability to obtain closed form expressions for (3.7) in the sense of (2.15) and (2.16) relies on the construction of the polynomials $\left\{R_{j}(x)\right\}$ so that they exhibit the skew orthogonality property

$$
\left\langle R_{2 j}, R_{2 k}\right\rangle^{A}=\left\langle R_{2 j+1}, R_{2 k+1}\right\rangle^{A}=0, \quad\left\langle R_{2 j}, R_{2 k+1}\right\rangle^{A}=r_{j} \delta_{j, k}
$$

where $\langle,\rangle^{A}$ denotes the skew inner product

$$
\langle f, g\rangle^{A}= \begin{cases}\int_{0}^{\infty} d y e^{-y / 2} \int_{y}^{\infty} d x e^{-x / 2} e^{A(x-y) / 2}(f(y) g(x)-g(y) f(x)), & \text { Laguerre } \\ \int_{-1}^{1} d y(1-y)^{(a-1) / 2} \int_{y}^{1} d x(1-x)^{(a-1) / 2}\left(\frac{1-x}{1-y}\right)^{-A / 2}(f(y) g(x)-g(y) f(x)), & \text { Jacobi }\end{cases}
$$

In the case $A=0$ the skew orthogonal polynomials in both the Laguerre and Jacobi cases are known in terms of an explicit series of Laguerre and Jacobi orthogonal polynomials respectively [34], as well as a more compact form involving derivatives and integrals of these bases [1]. Analogous explicit forms of the skew orthogonal polynomials are available in the limit $A \rightarrow-\infty$, when the PDFs (1.2) and (1.16) tend to certain PDFs of the form (1.15) corresponding to a symplectic symmetry. Regarding this latter point note that integration by parts shows

$$
\begin{aligned}
& \lim _{A \rightarrow-\infty}\left(\frac{A}{2}\right)^{2 n} \int_{X_{L}} d x_{1} \cdots d x_{2 n} \prod_{l=1}^{n} a_{l}\left(x_{2 l}\right) e^{-(1+A) x_{2 l-1} / 2} e^{-(1-A) x_{2 l} / 2} \prod_{1 \leq j<k \leq 2 n}\left(x_{j}-x_{k}\right) \\
& =\int_{\tilde{X}_{L}} d x_{2} d x_{4} \cdots d x_{2 n} \prod_{l=1}^{n} a_{l}\left(x_{2 l}\right) e^{-x_{2 l}} \prod_{1 \leq j<k \leq n}\left(x_{2 j}-x_{2 k}\right)^{4},
\end{aligned}
$$

where the $a_{l}$ are arbitrary, $X_{L}$ is the integration region (1.5), and $\tilde{X}_{L}$ the integration region $x_{2}>x_{4}>$ $\cdots>x_{2 n} \geq 0$, and

$$
\begin{aligned}
& \lim _{A \rightarrow-\infty}\left(\frac{A}{2}\right)^{2 n} \int_{X_{J}} d x_{1} \cdots d x_{2 n} \prod_{l=1}^{n} a_{l}\left(x_{2 l}\right)\left(1-x_{2 l-1}\right)^{(a-A-1) / 2}\left(1-x_{2 l}\right)^{(a+A-1) / 2} \prod_{1 \leq j<k \leq 2 n}\left(x_{j}-x_{k}\right) \\
& =\int_{\tilde{X}_{J}} d x_{2} d x_{4} \cdots d x_{2 n} \prod_{l=1}^{n} a_{l}\left(x_{2 l}\right)\left(1-x_{2 l}\right)^{a+1} \prod_{1 \leq j<k \leq n}\left(x_{2 j}-x_{2 k}\right)^{4},
\end{aligned}
$$

where the $a_{l}$ are arbitrary, and $X_{J}, \tilde{X}_{J}$ are the integration regions

$$
1>x_{1}>x_{2}>\cdots>x_{2 n}>-1, \quad 1>x_{2}>x_{4}>\cdots>x_{2 n}>-1
$$

respectively. Thus in the limit $A \rightarrow-\infty$ (1.4) reduces to the LSE with parameter $a=0$, while (1.16) reduces to the JSE with parameters $a \mapsto a+1$ and $b=0$. We see from (3.15) that in this limit the skew inner product takes the form

$$
\lim _{A \rightarrow-\infty}\left(\frac{A}{2}\right)^{2}\langle f, g\rangle^{A}= \begin{cases}\int_{0}^{\infty} d y e^{-y / 2}\left(f(y) \frac{d}{d y}\left(e^{-y / 2} g(y)\right)-(f \leftrightarrow g)\right), & \text { Laguerre } \\ \int_{-1}^{1} d y(1-y)^{(a+1) / 2}\left(f(y) \frac{d}{d y}\left((1-y)^{(a+1) / 2} g(y)\right)-(f \leftrightarrow g)\right), & \text { Jacobi }\end{cases}
$$

In keeping with the known results in the case $A=0$ and $A \rightarrow-\infty$, we find that the skew inner product admits skew orthogonal polynomials with compact expressions in terms of classical Laguerre and Jacobi polynomials. Their derivation relies on a special integration formula for the Laguerre polynomial $L_{k}(x)$ and Jacobi polynomial $P_{k}^{(a, 0)}(x)$, and the latter in turn rely on differentiation formulas for the same polynomials. 
Lemma 4. We have

$$
\begin{aligned}
\frac{d}{d t} L_{p}(t) & =-\sum_{l=0}^{p-1} L_{l}(t) \\
(1-t) \frac{d}{d t} P_{p}^{(a, 0)}(t) & =-p P_{p}^{(a, 0)}(t)+\sum_{l=0}^{p-1}(2 l+1+a)(-1)^{p-1-l} P_{l}^{(a, 0)}(t) .
\end{aligned}
$$

Proof. The formula (3.18) is an immediate consequence of the well known formula

$$
\frac{d}{d t}\left(L_{n}^{a}(t)-L_{n+1}^{a}(t)\right)=L_{n}^{a}(t)
$$

in the case $a=0$. To derive (3.19), we make use of the general formula

$$
\frac{d}{d t} P_{p}^{(a, b)}(t)=\frac{1}{2}(p+a+b+1) P_{p-1}^{(a+1, b+1)}(t)
$$

in the case $b=0$, then the general formula

$$
\left(n+\frac{1}{2}(a+b)+1\right)(1-t) P_{n}^{(a+1, b)}(t)=(n+a+1) P_{n}^{(a, b)}(t)-(n+1) P_{n+1}^{(a, b)}(t)
$$

again in the case $b=0$, to deduce that

$$
(1-t) \frac{d}{d t} P_{p}^{(a, 0)}(t)=\frac{p+1+a}{2 p+1+a}\left\{(p+a) P_{p-1}^{(a, 1)}(t)-p P_{p}^{(a, 1)}(t)\right\} .
$$

The formula (3.19) now follows from repeated use of the general formula

$$
P_{n}^{(a, b)}(t)=\frac{1}{n+a+b}\left((2 n+a+b) P_{n}^{(a, b-1)}(t)-(n+a) P_{n-1}^{(a, b)}(t)\right)
$$

in the case $b=1$.

Proposition 5. We have

$$
e^{(1-A) t / 2} \int_{t}^{\infty} e^{-(1-A) x / 2} L_{k}(x) d x=\sum_{p=0}^{k} c_{k p}^{L} L_{p}(t)
$$

where

$$
c_{k k}^{L}=\frac{2}{1-A}, \quad c_{k p}^{L}=(-1)^{p-k} \frac{4}{(1-A)^{2}}\left(\frac{1-A}{1+A}\right)^{p+1-k}, \quad(p=0, \ldots, k-1),
$$

and

$$
(1-t)^{-(a-A+1) / 2} \int_{t}^{1}(1-x)^{(a-A-1) / 2} P_{k}^{(a, 0)}(x) d x=\sum_{p=0}^{k} c_{k p}^{J} P_{p}^{(a, 0)}(t)
$$

where

$$
\begin{gathered}
c_{k k}^{J}=\frac{1}{k+(a-A+1) / 2}, \quad c_{k p}^{J}=(2 p+1+a) A_{p} B_{k},(p=0, \ldots, k-1), \\
A_{p}:=\frac{\Gamma(p+(a-A+1) / 2))}{\Gamma(p+(a+A+3) / 2))}, \quad B_{k}:=\frac{\Gamma(k+(a+A+1) / 2))}{\Gamma(k+(a-A+3) / 2))} .
\end{gathered}
$$

Proof. Consider first (3.20). Multiplying both sides by $e^{-(1-A) t / 2}$ and differentiating, making use of (3.18), gives

$$
-L_{k}(t)=-\frac{(1-A)}{2} \sum_{p=0}^{k} c_{k p}^{L} L_{k}(t)-\sum_{p=0}^{k} c_{k p}^{L} \sum_{l=0}^{p-1} L_{l}(t) .
$$


Equating coefficients of $L_{k}(t)$ gives $c_{k k}^{L}$ as stated in (3.21). Equating coefficients of $L_{p}(t)(p<k)$ gives

$$
0=-\frac{(1-A)}{2} c_{k p}^{L}-\sum_{j=p+1}^{k} c_{k j}^{L} \quad(p<k) .
$$

Replacing $p$ by $p-1$ and subtracting shows

$$
\delta_{k, p}=-\frac{(1-A)}{2} c_{k p-1}^{L}-\frac{(1+A)}{2} c_{k p}^{L} \quad(p \leq k)
$$

Solving this for $c_{k p}^{L}, p=k-1, k-2, \ldots, 0$ in order, making use of the known value of $c_{p p}^{L}$, completes the derivation of (3.21).

Consider now (3.22). Multiplying both sides by $(1-t)^{(a-A+1) / 2}$ and differentiating, making use of (3.19), gives

$$
\begin{aligned}
-P_{k}^{(a, 0)}(t)= & \sum_{p=0}^{k}-\left(p+\frac{1}{2}(a-A+1)\right) c_{k p}^{J} P_{p}^{(a, 0)}(t) \\
& +\sum_{p=0}^{k} \sum_{j=0}^{p-1} c_{k p}^{J}(2 j+1+a)(-1)^{p-1-j} P_{j}^{(a, 0)}(t) .
\end{aligned}
$$

Equating coefficients of $P_{p}^{(a, 0)}(t)$ gives

$$
-\delta_{p, k}=-\left(p+\frac{1}{2}(a-A+1)\right) c_{k p}^{J}+\sum_{l=p+1}^{k} c_{k l}^{J}(2 p+1+a)(-1)^{l-1-p},
$$

and replacing $p$ by $p-1$, multiplying by $(2 p+1+a) /(2 p-1+a)$, then adding to the original we obtain

$$
-\delta_{p, k}=\left(p+\frac{1}{2}(a+A+1)\right) c_{k p}^{J}-\frac{(p+(a-A-1) / 2)(2 p+1+a)}{2 p-1+a} c_{k p-1}^{J} .
$$

It follows from (3.27) with $p=k$ that the value of $c_{k k}^{J}$ is as stated in (3.23). From knowledge of $c_{k k}^{J}$ we can use (3.28) with $p=k-1, k-2, \ldots, 0$ in order to deduce the formula for $c_{k p}^{J}, p<k$ in (3.23).

An immediate corollary of Proposition 5 combined with the orthogonalities $(1.33)$ and (1.36) is the following integration formulas, which have direct use in the determination of the sought skew orthogonal polynomials.

Corollary 1. We have

$$
\int_{0}^{\infty} d t e^{-(1+A) t / 2} L_{j}(t) \int_{t}^{\infty} d x e^{-(1-A) x / 2} L_{k}(x)= \begin{cases}0, & j>k \\ c_{k j}^{L}, & j \leq k\end{cases}
$$

and

$$
\begin{aligned}
\int_{-1}^{1} d t & (1-t)^{(a-A-1) / 2} P_{j}^{(a, 0)}(t) \int_{t}^{1} d x(1-x)^{(a+A-1) / 2} P_{k}^{(a, 0)}(x) \\
= & \begin{cases}0, & j>k \\
\frac{2^{a+1}}{2 j+a+1} c_{k j}^{J}, & j \leq k\end{cases}
\end{aligned}
$$

The result of Corollary 1 implies that in both the Laguerre and Jacobi cases, we have identified a family of polynomials $\left\{p_{j}(x)\right\}\left(p_{j}(x)=L_{j}(x)\right.$ in the Laguerre case, and $p_{j}(x)=P_{j}^{(a, 0)}(x)$ in the Jacobi case) such that

$$
\left\langle p_{j}, p_{k}\right\rangle^{A}= \begin{cases}a_{j} b_{k}, & j<k \\ 0, & j=k \\ -a_{j} b_{k}, & j>k\end{cases}
$$


for certain $a_{j}, b_{k}$. We can use this special structure to construct the corresponding skew orthogonal polynomials as series in $\left\{p_{j}(x)\right\}$,

$$
R_{l}(x)=\sum_{j=0}^{l} \alpha_{l j} p_{j}(x), \quad \alpha_{l l}=1
$$

This is equivalent to finding a lower triangular matrix $T=\left[\alpha_{j k}\right]_{j, k=0,1, \ldots, 2 n-1}$ with 1's down the diagonal such that

$$
T\left[\alpha_{j k}\right] T^{t}=\left[\begin{array}{ccccccc}
0 & r_{0} & & & & & \\
-r_{0} & 0 & & & & & \\
& & 0 & r_{1} & & & \\
& & -r_{1} & 0 & & & \\
& & & & \ddots & & \\
& & & & & 0 & r_{n-1} \\
& & & & & -r_{n-1} & 0
\end{array}\right]
$$

where on the RHS all elements not explicitly shown are zero. An explicit solution to this problem is given by the following result.

Proposition 6. Let $p_{j}(x)(j=0,1, \ldots$,$) denote a polynomial of degree j$, and suppose the value of the skew product $\left\langle p_{j}, p_{k}\right\rangle$ factorizes as specified by (3.31). Then for $l$ even, with

$$
\alpha_{l 2 j+1}=-\frac{\prod_{\mu=j+1}^{l / 2-1} a_{2 \mu+1}}{\prod_{\mu=j+1}^{l / 2-1} a_{2 \mu}} \frac{\prod_{\mu=j+1}^{l / 2} b_{2 \mu}}{\prod_{\mu=j+1}^{l / 2} b_{2 \mu-1}}, \quad \alpha_{l 2 j}=\frac{\prod_{\mu=j}^{l / 2-1} a_{2 \mu+1}}{\prod_{\mu=j}^{l / 2-1} a_{2 \mu}} \frac{\prod_{\mu=j+1}^{l / 2} b_{2 \mu}}{\prod_{\mu=j+1}^{l / 2} b_{2 \mu-1}}, j \leq l / 2-1
$$

and for $l$ odd, with

$$
\alpha_{l l-1}=-\frac{b_{l}}{b_{l-1}}, \quad \alpha_{l j}=0, j \leq l-2
$$

the polynomials (3.39) exhibit the skew orthogonality property (3.14). The corresponding normalization is given by

$$
r_{(l-1) / 2}=a_{l-1} b_{l}
$$

Proof. Suppose first that $l$ is even, and consider

$$
\left\langle p_{j}, R_{l}\right\rangle^{A}, \quad j \leq l .
$$

Since $p_{j}$ can be written in terms of $\left\{R_{k}\right\}_{k=0, \ldots, l}$, it follows from (3.14) that

$$
\left\langle p_{j}, R_{l}\right\rangle^{A}=0 .
$$

But on the other hand, it follows from (3.32) and (3.31) that

$$
\left\langle p_{j}, R_{l}\right\rangle^{A}=a_{j} \sum_{\mu=j+1}^{l} \alpha_{l \mu} b_{\mu}-b_{j} \sum_{\mu=0}^{j-1} \alpha_{l \mu} a_{\mu} .
$$

Equating (3.37) and (3.38), and calling the resulting equation $C_{j}$ we see that forming

$$
-\frac{1}{a_{j}} C_{j}+\frac{1}{a_{j-1}} C_{j-1}
$$

gives the equation

$$
\alpha_{l j} b_{j}-\frac{b_{j-1}}{a_{j-1}} \sum_{\mu=0}^{j-2} \alpha_{l \mu} a_{\mu}+\frac{b_{j}}{a_{j}} \sum_{\mu=0}^{j-1} \alpha_{l \mu} a_{\mu}=0
$$


valid for $j=1,2, \ldots$ Substituting $j=1,2, \ldots$ in order in (3.39), we deduce that

$$
\begin{aligned}
& \alpha_{l 2 k+1}=-\frac{a_{2 k}}{a_{2 k+1}} \alpha_{l 2 k} \\
& \alpha_{l 2 k+2}=-\frac{b_{2 k+1}}{b_{2 k+2}} \alpha_{l 2 k+1}
\end{aligned}
$$

valid for $k=0,1, \ldots, l / 2-1$. Recalling the normalization $\alpha_{l l}=1$ we see that the recurrences reproduce (3.34).

Consider now the case $l$ odd. Then

$$
\left\langle p_{l-1}, R_{l}\right\rangle^{A}=r_{(l-1) / 2},
$$

and it follows from this and (3.38) with $j=l-1$ that

$$
r_{(l-1) / 2}=\left(a_{l-1} B_{l}-B_{l-1} \sum_{\mu=0}^{l-2} \alpha_{l \mu} a_{\mu}\right) .
$$

Making use of (3.39), which remains valid for $j<l-1$, we thus have

$$
-\frac{r_{(l-1) / 2}}{a_{l-1}}=\alpha_{l l-1} b_{l-1}-\frac{b_{l-2}}{a_{l-2}} \sum_{\mu=0}^{l-3} \alpha_{l \mu} a_{\mu}+\frac{b_{l-1}}{a_{l-1}} \sum_{\mu=0}^{l-2} \alpha_{l \mu} a_{\mu} .
$$

The fact that (3.39) remains valid for $j<l-1$ means that the equations (3.40) are again valid, this time for $2 k+1 \leq l-2$ in the first equation and $2 k+2 \leq l-3$ in the second equation. In particular, since we are assuming $l$ is odd, it follows from the first equation in (3.40) that

$$
\sum_{\mu=0}^{m} \alpha_{l \mu} a_{\mu}=0, \quad m=1,3, \ldots, l-2
$$

and thus (3.41) and (3.42) simplify to (3.36) and

$$
-\frac{r_{(l-1) / 2}}{a_{l-1}}=\alpha_{l l-1} b_{l-1}-\frac{b_{l-2}}{a_{l-2}} \alpha_{l l-3} a_{l-3}
$$

respectively. Substituting (3.36) in (3.43), we see that choosing $\alpha_{l l-3}=0$ implies the value of $\alpha_{l l-1}$ given in (3.35). The final equation in (3.35), $\alpha_{l j}=0, j \leq l-2$, follows from having chosen $\alpha_{l l-3}=0$ in (3.43) and the recurrences (3.40).

Examination of the above proof shows that for $l$ odd the value of $\alpha_{l l-1}$ is in fact completely arbitrary. This is because the skew orthogonal polynomials as specified by (3.14) are not unique. For a given family of polynomials $\left\{R_{j}(x)\right\}_{j=0,1, \ldots}$ satisfying (3.14), the family with

$$
R_{2 j+1}(x) \mapsto R_{2 j+1}(x)+\gamma_{j} R_{2 j}(x),
$$

$\gamma_{j}$ arbitrary, also satisfy (3.14). This non-uniqueness underlies the arbitrariness of $\alpha_{l l-1}$; the choice made in (3.35) leads to the simplest result in that with this choice we then have $\alpha_{l j}=0$ for all $j \leq l-2$.

Inserting the explicit value of $a_{j} b_{k}$ in (3.31) from Corollary 1, we get from Proposition 6 the following explicit formulas for the skew orthogonal polynomials in the Laguerre and Jacobi cases.

Corollary 2. The polynomials

$$
\begin{aligned}
R_{2 l-1}^{(L)}(x) & =L_{2 l-1}(x)-\frac{A+1}{A-1} L_{2 l-2}(x) \\
R_{2 l}^{(L)}(x) & =\sum_{j=0}^{l} L_{2 j}(x)-\frac{A+1}{A-1} \sum_{j=0}^{l-1} L_{2 j+1}(x)
\end{aligned}
$$


are skew orthogonal with respect to the skew inner product (3.15) in the Laguerre case, while the polynomials

$$
\begin{aligned}
R_{2 l-1}^{(J)}(x) & =P_{2 l-1}^{(a, 0)}(x)-\frac{2 l+(a-A-3) / 2}{2 l+(a-A-1) / 2} P_{2 l-2}^{(a, 0)}(x) \\
R_{2 l}^{(J)}(x) & =P_{2 l}^{(a, 0)}(x)+\sum_{j=0}^{2 l-1}(-1)^{j} \frac{j+\left(a-(-1)^{j} A+1\right) / 2}{2 l+(a-A+1) / 2} P_{j}^{(a, 0)}(x)
\end{aligned}
$$

are skew orthogonal with respect to the skew inner product (3.15) in the Jacobi case. The corresponding normalizations are

$$
r_{l}^{(L)}=-\frac{4}{(1-A)^{2}}, \quad r_{l}^{(J)}=\frac{2^{a+1}}{(2 l+(a-A+3) / 2)(2 l+(a-A+1) / 2)} .
$$

Even though the matrix elements (3.7) for the correlations (1.27) explicitly depend on $\left\{R_{j}(x)\right\}$, we will not directly make use of the formulas (3.44) and (3.45) in our subsequent simplification of (3.7). Rather we will make use of these formulas to evaluate the indefinite integral

$$
\int_{y}^{\infty} w(x) \kappa(x, y) R_{k}(x) d x
$$

which will then be used in (3.7). With $R_{k}(x)$ replaced by $p_{k}(x)$, this integral is given by (3.20) in the Laguerre case, and (3.22) in the Jacobi case. Using the notation of the RHS of (3.31), and introducing the additional symbol $\tilde{a}_{k}$, these results can be combined into the single formula

$$
\frac{1}{\tilde{w}(y)} \int_{y}^{\infty} w(x) \kappa(x, y) p_{k}(x) d x=\frac{\tilde{a}_{k}}{h_{k}} p_{k}(y)+\sum_{j=0}^{k-1} \frac{a_{j} b_{k}}{h_{j}} p_{j}(y)
$$

where

$$
\tilde{w}(x)=\left\{\begin{array}{ll}
w(x), & \text { Laguerre } \\
(1-x) w(x), & \text { Jacobi }
\end{array} h_{j}:=\int_{-\infty}^{\infty} w(x) \tilde{w}(x)\left(p_{j}(x)\right)^{2} d x .\right.
$$

We can use (3.48) together with the result of Proposition 6 to evaluate (3.47).

Proposition 7. Let $\left\{R_{j}(x)\right\}$ be given by (3.32), with the $\alpha_{l j}$ therein specified by Proposition 0 . Furthermore, assume the integral evaluation (3.48). Then

$$
\frac{1}{\tilde{w}(y)} \int_{y}^{\infty} w(x) \kappa(x, y) R_{k}(x) d x=\sum_{l=0}^{k} u_{k l} p_{l}(y)
$$

where for $k$ even

$$
u_{k l}= \begin{cases}\frac{\alpha_{k l} \tilde{a}_{l}}{h_{l}}+\frac{a_{l} \alpha_{k l+1} b_{l+1}}{h_{l}}, & l \text { odd } \\ \frac{\alpha_{k l} \tilde{a}_{l}}{h_{l}}, & l \text { even }\end{cases}
$$

while for $k$ odd

$$
u_{k l}= \begin{cases}\frac{\tilde{a}_{k}}{h_{k}}, & l=k \\ \frac{\tilde{a}_{k} \alpha_{k k-1}}{h_{k}}+\frac{a_{k-1} b_{k}}{h_{k}}, & l=k-1 \\ 0, & \text { otherwise }\end{cases}
$$


Proof. $\quad$ Substituting (3.32) in (3.47) and making use of (3.48) gives

$$
\frac{1}{\tilde{w}(y)} \int_{y}^{\infty} w(x) \kappa(x, y) R_{k}(x) d x=\sum_{j=0}^{k} \frac{\alpha_{k j} \tilde{a}_{j}}{h_{j}} p_{j}(y)+\sum_{j=0}^{k} \alpha_{k j} \sum_{\mu=0}^{j-1} \frac{a_{\mu} b_{j}}{h_{\mu}} p_{\mu}(y) .
$$

The coefficient of $p_{l}(y)$ in the above expression is

$$
\frac{\alpha_{k l} \tilde{a}_{l}}{h_{l}}+\frac{a_{l}}{h_{l}} \sum_{j=l+1}^{k} \alpha_{k j} b_{j} .
$$

But for $k$ even, the second formula in (3.40) shows that we get cancellation in pairs in the above summation, and (3.50) results. For $k$ odd, we see from (3.35) that the summation in (3.52) vanishes for $l<k-1$, and that so too does the first term. The terms which remain give (3.51).

We now substitute the particular values of the quantities $\tilde{a}_{l}, b_{j}, a_{k}$ implied by (3.21) and (3.22), together with the explicit formulas for $\alpha_{k l}$ implied by (3.44) and (3.45), and the normalizations (1.33) and (1.36), in (3.50) and (3.51). This shows that in both the Laguerre and Jacobi cases the coefficients $u_{k l}$, up to a sign, are independent of $l$. Furthermore, in the case $k$ even we can identify the resulting series as a linear combination of

$$
\frac{1}{\tilde{w}(y)} \int_{y}^{\infty} w(x) p_{k}(x) d x \quad \text { and } \quad p_{k}(x),
$$

or alternatively as a linear combination of

$$
\frac{1}{\tilde{w}(y)} \int_{y}^{\infty} w(x) p_{k+1}(x) d x \quad \text { and } \quad p_{k+1}(x) .
$$

Corollary 3. The polynomials (3.44) have the properties that

$$
e^{y / 2} \int_{y}^{\infty} e^{-t / 2} e^{A(t-y) / 2} R_{2 k+1}^{(L)}(t) d t=\frac{2}{1-A}\left(-L_{2 k+1}(y)+L_{2 k}(y)\right)
$$

and

$$
\begin{aligned}
e^{y / 2} \int_{y}^{\infty} e^{-t / 2} e^{A(t-y) / 2} R_{2 k}^{(L)}(t) d t & =\frac{2}{1-A}\left(\sum_{j=0}^{k} L_{2 j}(y)-\sum_{j=1}^{k} L_{2 j-1}(y)\right) \\
& =\frac{1}{1-A} L_{2 k}(y)+\frac{e^{y / 2}}{2(1-A)} \int_{y}^{\infty} e^{-s / 2} L_{2 k}(s) d s \\
& =\frac{1}{1-A} L_{2 k+1}(y)-\frac{e^{y / 2}}{2(1-A)} \int_{y}^{\infty} e^{-s / 2} L_{2 k+1}(s) d s
\end{aligned}
$$

while the polynomials (3.45) have the properties that

$$
(1-y)^{-(a+1) / 2} \int_{y}^{1}(1-t)^{(a-1) / 2}\left(\frac{1-t}{1-y}\right)^{-A / 2} R_{2 k+1}^{(J)}(t) d t=\frac{1}{2 k+(a-A+3) / 2}\left(P_{2 k+1}^{(a, 0)}(y)+P_{2 k}^{(a, 0)}(y)\right)
$$

and

$$
\begin{aligned}
(1- & y)^{-(a+1) / 2} \int_{y}^{1}(1-t)^{(a-1) / 2}\left(\frac{1-t}{1-y}\right)^{-A / 2} R_{2 k}^{(J)}(t) d t \\
& =\frac{1}{2 k+(a-A+1) / 2} \sum_{l=0}^{2 k} P_{l}^{(a, 0)}(y) \\
& =\frac{1}{2 k+(a-A+1) / 2}\left(\frac{1}{2} P_{2 k}^{(a, 0)}(y)+\frac{2 k+(a+1) / 2}{2(1-y)^{(a+1) / 2}} \int_{y}^{1}(1-t)^{(a-1) / 2} P_{2 k}^{(a, 0)}(t) d t\right) \\
& =\frac{1}{2 k+(a-A+1) / 2}\left(-\frac{1}{2} P_{2 k+1}^{(a, 0)}(y)+\frac{2 k+1+(a+1) / 2}{2(1-y)^{(a+1) / 2}} \int_{y}^{1}(1-t)^{(a-1) / 2} P_{2 k+1}^{(a, 0)}(t) d t\right)
\end{aligned}
$$


Proof. The series expansions follow immediately upon making the stated substitutions. To obtain the integral formulas, we substitute for the summations in (3.54) and (3.56) according to their value implied by $(3.20)$ and $(3.22$ respectively.

We remark that the series of Laguerre and Jacobi polynomials in (3.53)-3.56) can each, according to the results (3.44) and (3.45), be identified with $\left.R_{j}(y)\right|_{A \rightarrow-\infty}$ for $j=2 k+1$ or $j=2 k$ as appropriate.

\subsection{Summation formulas - the even-even block}

The skew orthogonality property (3.14) implies the matrix $M_{j k}$ as specified by (3.6) is equal to -1 times the RHS of (3.33). Thus

$$
M_{j k}^{-t}= \begin{cases}0, & (j, k) \neq(2 l, 2 l+1) \text { or }(2 l+1,2 l) \\ -r_{l}^{-1}, & (j, k)=(2 l, 2 l+1) \quad(l=0, \ldots, n-1) \\ r_{l}^{-1}, & (j, k)=(2 l+1,2 l) \quad(l=0, \ldots, n-1)\end{cases}
$$

and so the double summations in (3.7) all collapse to single summations. In particular, with the entry in row $s$, column $s^{\prime}$ of the matrix $f_{a b}(a, b=$ e or o $)$ denoted $f_{a b}^{s s^{\prime}}$, we have that

$$
f_{\mathrm{ee}}^{12}\left(y, y^{\prime}\right)=\sum_{j=0}^{n-1} \frac{1}{r_{j}}\left(\Phi_{2 j}^{\mathrm{e}}(y) \Phi_{2 j+1}^{\mathrm{e}}\left(y^{\prime}\right)-\Phi_{2 j}^{\mathrm{e}}\left(y^{\prime}\right) \Phi_{2 j+1}^{\mathrm{e}}(y)\right),
$$

where $\Phi_{j}^{\mathrm{e}}$ is defined by (1.28) and (1.29) in the Laguerre and Jacobi cases respectively. The latter indefinite integrals are precisely those occurring in Corollary 3 . Using this result, $f_{\mathrm{ee}}^{12}$ can be expressed in terms of the functions $K_{2 n}^{L}$ in the Laguerre case and $K_{2 n}^{J}$ in the Jacobi case.

Proposition 8. In the Laguerre case

$$
f_{\mathrm{ee}}^{12}\left(y, y^{\prime}\right)=\frac{1}{4}\left(\int_{y}^{\infty} K_{2 n}^{L}\left(y^{\prime}, t\right) d t-\int_{y^{\prime}}^{\infty} K_{2 n}^{L}(y, t) d t\right)
$$

while in the Jacobi case

$$
f_{\mathrm{ee}}^{12}\left(y, y^{\prime}\right)=\frac{1}{4}\left(\left(1-y^{\prime}\right) \int_{y}^{1} K_{2 n}^{J}\left(y^{\prime}, t\right) d t-(1-y) \int_{y^{\prime}}^{1} K_{2 n}^{J}(y, t) d t\right) .
$$

Proof. Consider the Jacobi case. Substituting for $r_{j}^{(J)}$ using (3.46) and substituting for $\Phi_{2 k+1}\left(y^{\prime}\right)$ using (3.55) we see that

$$
\begin{aligned}
& f_{\mathrm{ee}}^{12}\left(y, y^{\prime}\right)=\frac{1}{2^{a+1}}(1-y)^{(a+1) / 2}\left(1-y^{\prime}\right)^{(a+1) / 2} \\
& \quad \times \sum_{j=0}^{n-1}(2 j+(a-A+1) / 2)\left(P_{2 j+1}^{(a, 0)}\left(y^{\prime}\right) \Phi_{2 j}(y)+P_{2 j}^{(a, 0)}\left(y^{\prime}\right) \Phi_{2 j}(y)\right)-\left(y \leftrightarrow y^{\prime}\right) .
\end{aligned}
$$

In the product $P_{2 j+1}^{(a, 0)}\left(y^{\prime}\right) \Phi_{2 j}(y)$ we substitute for $(1-y)^{(a+1) / 2} \Phi_{2 j}(y)$ using the third equality in (3.56), while in the product $P_{2 j+1}^{(a, 0)}\left(y^{\prime}\right) \Phi_{2 j}(y)$ we substitute for $(1-y)^{(a+1) / 2} \Phi_{2 j}(y)$ using the second equality in (3.56). Straightforward simplification and comparison with (1.35), taking note of (1.36), gives (3.60). The Laguerre case is similar.

The single sum form of the other matrix elements of $f_{\mathrm{ee}}$ are

$$
f_{\mathrm{ee}}^{11}\left(y, y^{\prime}\right)=f_{\mathrm{ee}}^{22}\left(y^{\prime}, y\right)=-\sum_{j=0}^{n-1} \frac{w\left(y^{\prime}\right)}{r_{j}}\left(\Phi_{2 j}^{\mathrm{e}}(y) R_{2 j+1}\left(y^{\prime}\right)-\Phi_{2 j+1}^{\mathrm{e}}(y) R_{2 j}\left(y^{\prime}\right)\right),
$$




$$
f_{\mathrm{ee}}^{21}\left(y, y^{\prime}\right)=-\sum_{j=0}^{n-1} \frac{w(y) w\left(y^{\prime}\right)}{r_{j}}\left(R_{2 j}(y) R_{2 j+1}\left(y^{\prime}\right)-R_{2 j+1}(y) R_{2 j}\left(y^{\prime}\right)\right) .
$$

Noting from (1.28) that in the Laguerre case

$$
\frac{A}{2} \Phi_{j}^{\mathrm{e}}(x)+\frac{d}{d x} \Phi_{j}^{\mathrm{e}}(x)=-e^{-x / 2} R_{j}(x)
$$

and from $(\overline{1.29})$ that in the Jacobi case

$$
\frac{A}{2(1-x)} \Phi_{j}^{\mathrm{e}}(x)+\frac{d}{d x} \Phi_{j}^{\mathrm{e}}(x)=-(1-x)^{(a-1) / 2} R_{j}(x),
$$

we see that all the quantities $f_{\mathrm{ee}}^{11}, f_{\mathrm{ee}}^{22}$ and $f_{\mathrm{ee}}^{21}$ can be expressed in terms of $f_{\mathrm{ee}}^{12}$ and thus $K_{2 n}^{L}$ and $K_{2 n}^{J}$.

Proposition 9. In the Laguerre case

$$
f_{\mathrm{ee}}^{11}\left(y, y^{\prime}\right)=f_{\mathrm{ee}}^{22}\left(y^{\prime}, y\right)=\left(\frac{A}{2}+\frac{\partial}{\partial y^{\prime}}\right) f_{\mathrm{ee}}^{12}\left(y, y^{\prime}\right), \quad f_{\mathrm{ee}}^{21}\left(y, y^{\prime}\right)=-\left(\frac{A}{2}+\frac{\partial}{\partial y}\right)\left(\frac{A}{2}+\frac{\partial}{\partial y^{\prime}}\right) f_{\mathrm{ee}}^{12}\left(y, y^{\prime}\right)
$$

while in the Jacobi case

$$
\begin{gathered}
f_{\mathrm{ee}}^{11}\left(y, y^{\prime}\right)=f_{\mathrm{ee}}^{22}\left(y^{\prime}, y\right)=\left(\frac{A}{2\left(1-y^{\prime}\right)}+\frac{\partial}{\partial y^{\prime}}\right) f_{\mathrm{ee}}^{12}\left(y, y^{\prime}\right) \\
f_{\mathrm{ee}}^{21}\left(y, y^{\prime}\right)=-\left(\frac{A}{2(1-y)}+\frac{\partial}{\partial y}\right)\left(\frac{A}{2\left(1-y^{\prime}\right)}+\frac{\partial}{\partial y^{\prime}}\right) f_{\mathrm{ee}}^{12}\left(y, y^{\prime}\right) .
\end{gathered}
$$

The $(0, k)$-point parity aware correlation, or equivalently the $k$-point correlation for the even labelled coordinates, is according to (1.24) equal to $q \operatorname{det}\left[f_{\mathrm{ee}}\left(y_{j}, y_{l}\right)\right]_{j, l=1, \ldots, k}$. By performing elementary row and column operations, making sure to conserve the self dual structure (3.2), we see from Proposition 9 that in both the Laguerre and Jacobi cases all terms dependent on the parameter $A$ can be eliminated, leaving as the final expression

$$
\rho_{(0, k)}\left(y_{1}, \ldots, y_{k}\right)=\operatorname{qdet}\left[\begin{array}{cc}
\frac{\partial}{\partial y_{l}} f_{\mathrm{ee}}^{12}\left(y_{j}, y_{l}\right) & f_{\mathrm{ee}}^{12}\left(y_{j}, y_{l}\right) \\
-\frac{\partial^{2}}{\partial y_{j} \partial y_{l}} f_{\mathrm{ee}}^{12}\left(y_{j}, y_{l}\right) & \frac{\partial}{\partial y_{j}} f_{\mathrm{ee}}^{12}\left(y_{l}, y_{j}\right)
\end{array}\right]_{j, l=1, \ldots, k} .
$$

The independence on $A$ is required by the identity (1.19), valid for the Laguerre and Jacobi weights in (1.20). Moreover, this $k$-point correlation must agree with the $k$-point correlation for the LSE with parameter $a=0$ in the Laguerre case, and for the JSE with parameters $a \mapsto a+1, b=0$ in the Jacobi case (see (3.106) and Section 5.2).

\subsection{Summation formulas - the parity blind case}

The matrix element (3.61) is fundamental with respect to all other matrix elements in (3.7) and (3.13) in that each of the latter can be constructed from (3.61) by integration. The evaluation (3.59) in the Laguerre case and (3.63) in the Jacobi case of (3.61) then allows us to express all matrix elements in (3.7) and (3.13) in terms of $K_{2 n}^{L}$ and $K_{2 n}^{J}$. In this subsection we will undertake this program for the matrix elements of (3.13). Formulas for the matrix elements of the blocks $f_{\mathrm{eo}}, f_{\mathrm{oe}}$ and $f_{\mathrm{oo}}$ in (3.17), obtained using knowledge of the evaluation of the matrix elements for the block $f_{\text {ee }}$ given in subsection 3.3 and the evaluation of the matrix elements of (3.13) to be given in this subsection, will be presented in the next subsection. 
Recalling the definition of $f_{\mathrm{ee}}^{21}\left(y, y^{\prime}\right)$ from (3.7), and the definition (3.11) of $\left(\epsilon \cdot R_{k}\right)$, we see that the matrix elements of $f(x, y)$ in $(3.13)$ can be written in terms of $f_{\mathrm{ee}}^{21}$ according to

$$
\begin{aligned}
& f^{11}(x, y)=f^{22}(y, x)=\int_{-\infty}^{\infty} \epsilon(y, t) f_{\mathrm{ee}}^{21}(x, t) d t \\
& f^{12}(x, y)=-f_{\mathrm{ee}}^{21}(x, y) \\
& f^{21}(x, y)=-\epsilon(x, y)+\int_{-\infty}^{\infty} d s \epsilon(x, s) \int_{-\infty}^{\infty} d t \epsilon(y, t) f_{\mathrm{ee}}^{21}(s, t)
\end{aligned}
$$

These formulas can be made more explicit. For this purpose use will be made of the following formulas.

Lemma 10. We have

$$
-\int_{y}^{\infty} K_{2 n}^{L}(0, u) d u=\int_{0}^{\infty} K_{2 n}^{L}(y, u) d u
$$

and

$$
-\int_{y}^{1} K_{2 n}^{J}(-1, u) d u=\frac{(1-y)}{2} \int_{-1}^{1} K_{2 n}^{J}(y, u) d u
$$

Proof. It follows from (3.20) with $t=0, A=0$, and the evaluation formula

$$
L_{p}(0)=1
$$

that

$$
\int_{0}^{\infty} e^{-t / 2} L_{p}(t) d t=2(-1)^{p}
$$

Recalling the definition (1.32) of $K_{n}^{L}$ we thus have

$$
\int_{0}^{\infty} K_{2 n}^{L}(y, u) d u=2 e^{-y / 2} \sum_{p=0}^{2 n-1}(-1)^{p} L_{p}(y) .
$$

Also, from (3.20) with $A=0$ we have

$$
e^{t / 2} \int_{t}^{\infty} e^{-x / 2} L_{k}(x) d x=2 L_{k}(t)+4(-1)^{k} \sum_{p=0}^{k-1}(-1)^{p} L_{p}(t) .
$$

It follows from this integration formula and (3.70) that the LHS of (3.68) reduces to the RHS of (3.71).

To establish (3.69), we note that it follows from (3.22) with $t=0, A=0$, and the evaluation formula

$$
P_{l}^{(a, 0)}(0)=(-1)^{l}
$$

that

$$
\int_{-1}^{1}(1-x)^{(a-1) / 2} P_{k}^{(a, 0)}(x) d x=\frac{2^{(a+1) / 2}}{k+(a+1) / 2} .
$$

It follows from this and $(1.35)$ that

$$
\int_{-1}^{1} K_{2 n}^{J}(t, y) d t=2^{-(a-1) / 2}(1-y)^{(a-1) / 2} \sum_{j=0}^{2 n-1} P_{j}^{(a, 0)}(y) .
$$

Regarding the LHS of (3.69), we note from (3.22) with $A=0$ that

$$
(1-t)^{-(a+1) / 2} \int_{t}^{1}(1-x)^{(a-1) / 2} P_{k}^{(a, 0)}(x) d x=\frac{1}{k+(a+1) / 2}\left\{P_{k}(t)+2 \sum_{l=0}^{k-1} P_{l}(t)\right\} .
$$

This integration formula together with (3.73) shows that the LHS of (3.69) reduces to the RHS of (3.75). 
Proposition 11. In the Laguerre case

$$
\begin{aligned}
f^{22}(x, y)= & \frac{1}{2} K_{2 n}^{L}(x, y)-\frac{1}{2} \frac{\partial}{\partial y} \int_{0}^{x} e^{A(x-t) / 2} K_{2 n}^{L}(t, y) d t \\
& -\frac{A}{4} \int_{0}^{x} d t e^{A(x-t) / 2} \int_{y}^{\infty} d u \frac{\partial}{\partial t} K_{2 n}^{L}(u, t)+\frac{A}{4} e^{A x / 2} \int_{0}^{\infty} K_{2 n}^{L}(y, u) d u \\
f^{12}(x, y)= & \frac{1}{4}\left(\frac{A}{2}+\frac{\partial}{\partial x}\right)\left(\frac{A}{2}+\frac{\partial}{\partial y}\right)\left\{\int_{x}^{\infty} K_{2 n}^{L}(y, t) d t\right. \\
& \left.-\int_{y}^{\infty} K_{2 n}^{L}(x, t) d t\right\} \\
f^{21}(x, y)= & -e^{A|x-y| / 2} \operatorname{sgn}(x-y)-\left\{\int_{0}^{y} e^{A(y-t) / 2} K_{2 n}^{L}(x, t) d t\right. \\
& \left.-\int_{0}^{x} e^{A(x-t) / 2} K_{2 n}^{L}(y, t) d t\right\}
\end{aligned}
$$

while in the Jacobi case

$$
\begin{aligned}
f^{22}(x, y)= & \frac{1}{2}(1-x) K_{2 n}^{J}(x, y)-\frac{1}{2}\left\{(1-y) \frac{\partial}{\partial y}-1\right\} \int_{-1}^{x}\left(\frac{1-t}{1-x}\right)^{A / 2} K_{2 n}^{J}(t, y) d t \\
& +\frac{A}{4(1-y)} \int_{-1}^{x} d t\left(\frac{1-t}{1-x}\right)^{A / 2} \int_{y}^{1} d u\left\{1-(1-t) \frac{\partial}{\partial t}\right\} K_{2 n}^{J}(u, t) \\
& +\frac{A}{4(1-y)}\left(\frac{2}{1-x}\right)^{A / 2} \int_{-1}^{1} K_{2 n}^{J}(y, u) d u \\
f^{12}(x, y)= & \frac{1}{4}\left(\frac{A}{2(1-x)}+\frac{\partial}{\partial x}\right)\left(\frac{A}{2(1-y)}+\frac{\partial}{\partial y}\right)\left\{(1-y) \int_{x}^{1} K_{2 n}^{J}(y, t) d t\right. \\
& \left.-(1-x) \int_{y}^{1} K_{2 n}^{J}(x, t) d t\right\} \\
f^{21}(x, y)= & -\left(\frac{1-x}{1-y}\right)^{-A \operatorname{sgn}(x-y) / 2} \operatorname{sgn}(x-y)-\left\{(1-x) \int_{-1}^{y}\left(\frac{1-t}{1-y}\right)^{A / 2} K_{2 n}^{J}(x, t) d t\right. \\
& \left.-(1-y) \int_{-1}^{x}\left(\frac{1-t}{1-x}\right)^{A / 2} K_{2 n}^{J}(y, t) d t\right\}
\end{aligned}
$$

Proof. Consider first the Laguerre case. The formula (3.78) follows immediately from (3.66), upon substituting (3.59) in the second formula of (3.62). In preparation for deriving (3.77), we note that the last substitution, after computation of the corresponding derivatives where possible, yields

$$
\begin{aligned}
f_{\mathrm{ee}}^{21}\left(y, y^{\prime}\right)= & \frac{1}{4}\left(\frac{A}{2}\right)^{2}\left\{\int_{y^{\prime}}^{\infty} K_{2 n}^{L}(y, t) d t+\int_{y}^{\infty} K_{2 n}^{L}\left(y^{\prime}, t\right) d t\right\} \\
& +\frac{A}{8}\left\{\int_{y^{\prime}}^{\infty} \frac{\partial}{\partial y} K_{2 n}^{L}(y, t) d t-\int_{y}^{\infty} \frac{\partial}{\partial y^{\prime}} K_{2 n}^{L}\left(y^{\prime}, t\right) d t\right\} \\
& +\frac{1}{4}\left\{\frac{\partial}{\partial y}-\frac{\partial}{\partial y^{\prime}}\right\} K_{2 n}^{L}\left(y, y^{\prime}\right) .
\end{aligned}
$$

Thus there are three distinct terms which must be substituted in (3.65).

Substituting the $A$ independent term from (3.83) in (3.65) and integrating by parts gives the contribution

$$
\begin{aligned}
& \frac{1}{4}\left\{-e^{A x / 2} K_{2 n}^{L}(0, y)+2 K_{2 n}^{L}(x, y)+\frac{A}{2} \int_{0}^{\infty} e^{A|x-t| / 2} K_{2 n}^{L}(t, y) d t\right. \\
& \left.\quad-2 \frac{\partial}{\partial y} \int_{0}^{x} e^{A(x-t) / 2} K_{2 n}^{L}(t, y) d t+\frac{\partial}{\partial y} \int_{0}^{\infty} e^{A|x-t| / 2} K_{2 n}^{L}(t, y) d t\right\} .
\end{aligned}
$$


The expression obtained by substituting the term proportional to $A$ from (3.83) in (3.65) can be simplified by integrating by parts immediately in one term, while in the other first making use of the identity

$$
-\frac{A}{2} \operatorname{sgn}(x-t) e^{A|x-t| / 2}=\frac{d}{d t} e^{A|x-t| / 2}
$$

and then integrating by parts. Doing this allows the contribution to be written

$$
\begin{aligned}
& \frac{1}{4} e^{A x / 2} \int_{0}^{\infty} \frac{\partial}{\partial y} K_{2 n}^{L}(t, y) d t-\frac{1}{4} \int_{0}^{\infty} e^{A|x-t| / 2} \frac{\partial}{\partial y} K_{2 n}^{L}(t, y) d t \\
& -\frac{A}{4} \int_{0}^{x} d t e^{A|x-t| / 2} \int_{y}^{\infty} d u \frac{\partial}{\partial t} K_{2 n}^{L}(u, t) \\
& +\frac{A}{8} \int_{0}^{\infty} d t e^{A|x-t| / 2} \int_{y}^{\infty} d u \frac{\partial}{\partial t} K_{2 n}^{L}(u, t) .
\end{aligned}
$$

Simplifying the contribution to (3.65) from the term proportional to $A^{2}$ from 3.83 in the same way gives for the final term

$$
\begin{aligned}
& \frac{A}{8}\left\{-e^{A x / 2} \int_{y}^{\infty} K_{2 n}^{L}(0, u) d u-\int_{0}^{\infty} d t e^{A|x-t| / 2} \int_{y}^{\infty} d u \frac{\partial}{\partial t} K_{2 n}^{L}(u, t)\right. \\
& \left.\quad+e^{A x / 2} \int_{0}^{\infty} K_{2 n}^{L}(y, u) d u-\int_{0}^{\infty} e^{A|x-t| / 2} K_{2 n}^{L}(y, t) d t\right\}
\end{aligned}
$$

Adding together (3.84)-(3.87) and simplifying using (3.68) and the formula

$$
K_{2 n}^{L}(0, u)=\int_{0}^{\infty} \frac{\partial}{\partial y} K_{2 n}^{L}(y, u) d u,
$$

which follows from (3.68) by differentiation, we obtain (3.77). To derive (3.79), we first note from (3.65) that 3.67 ) can be rewritten

$$
f^{21}(x, y)=\epsilon(x, y)+\int_{-\infty}^{\infty} d s \epsilon(x, s) f^{11}(s, y)
$$

and then substitute (3.77) in this formula. Simplification along the same lines as that detailed above gives (3.79).

Next we turn our attention to the Jacobi case. Substituting (3.60) in (3.63), then substituting the result in (3.66) gives (3.81). The former substitution, with the derivatives computed where possible, yields

$$
\begin{aligned}
f_{\mathrm{ee}}^{21}\left(y, y^{\prime}\right)= & \frac{A^{2}}{16}\left\{\frac{1}{1-y^{\prime}} \int_{y^{\prime}}^{1} K_{2 n}^{J}(y, t) d t-\frac{1}{1-y} \int_{y}^{1} K_{2 n}^{J}\left(y^{\prime}, t\right) d t\right\} \\
& +\frac{A}{8}\left\{\frac{1}{1-y^{\prime}} \int_{y^{\prime}}^{1}\left(1-(1-y) \frac{\partial}{\partial y}\right) K_{2 n}^{J}(y, t) d t-\frac{1}{1-y} \int_{y}^{1}\left(1-\left(1-y^{\prime}\right) \frac{\partial}{\partial y^{\prime}}\right) K_{2 n}^{J}\left(y^{\prime}, t\right) d t\right\} \\
& -\frac{1}{4}\left\{\left((1-y) \frac{\partial}{\partial y}-1\right)-\left(\left(1-y^{\prime}\right) \frac{\partial}{\partial y^{\prime}}-1\right)\right\} K_{2 n}^{J}\left(y, y^{\prime}\right) .
\end{aligned}
$$

This has an analogous structure to (3.83), consisting of three distinct terms which must be substituted in 3.65 .

Substituting the $A$ dependent term from 3.89 ) in (3.65) and integrating by parts gives the contribu- 
tion

$$
\begin{aligned}
& \frac{1}{2}\left(\frac{2}{1-x}\right)^{A / 2} K_{2 n}^{J}(-1, y)+\frac{1}{2} \int_{-1}^{x}\left((1-t) \frac{\partial}{\partial t}-1\right)\left(\frac{1-t}{1-x}\right)^{A / 2} K_{2 n}^{J}(t, y) d t \\
& +\frac{A}{8} \int_{-1}^{1}\left(\frac{1-t}{1-x}\right)^{A \operatorname{sgn}(x-t) / 2} K_{2 n}^{J}(t, y) d t \\
& -\frac{1}{2}\left((1-y) \frac{\partial}{\partial y}-1\right) \int_{-1}^{x}\left(\frac{1-t}{1-x}\right)^{A / 2} K_{2 n}^{J}(t, y) d t \\
& +\frac{1}{4}\left((1-y) \frac{\partial}{\partial y}-1\right) \int_{-1}^{1}\left(\frac{1-t}{1-x}\right)^{A \operatorname{sgn}(\mathrm{x}-\mathrm{t}) / 2} K_{2 n}^{J}(t, y) d t .
\end{aligned}
$$

For the term proportional to $A$ in (3.89), as well as using direct integration by parts to simplify its contribution to (3.65) in one of the terms, we make use of the identity

$$
-\frac{A}{2} \operatorname{sgn}(x-t) \frac{1}{1-t}(1-t)^{A \operatorname{sgn}(x-t) / 2}=\frac{d}{d t}(1-t)^{-A \operatorname{sgn}(x-t) / 2}
$$

(c.f. (3.85)) and then integrate by parts in the other. This contribution is then found to equal

$$
\begin{aligned}
& -\frac{1}{4}\left(\frac{2}{1-x}\right)^{A / 2} \int_{-1}^{x}\left(1-(1-y) \frac{\partial}{\partial y}\right) K_{2 n}^{J}(t, y) d t \\
& +\frac{1}{4} \int_{-1}^{1}\left(\frac{1-t}{1-x}\right)^{A \operatorname{sign}(x-t) / 2}\left(1-(1-y) \frac{\partial}{\partial y}\right) K_{2 n}^{J}(t, y) d t \\
& +\frac{A}{4} \frac{1}{1-y} \int_{-1}^{x}\left(\frac{1-t}{1-x}\right)^{A / 2}\left(\int_{y}^{1}\left(1-(1-t) \frac{\partial}{\partial t}\right) K_{2 n}^{J}(t, u) d u\right) d t \\
& -\frac{A}{8} \frac{1}{1-y} \int_{-1}^{1}\left(\frac{1-t}{1-x}\right)^{A \operatorname{sgn}(x-t) / 2}\left(\int_{y}^{1}\left(1-(1-t) \frac{\partial}{\partial t}\right) K_{2 n}^{J}(t, u) d u\right) d t
\end{aligned}
$$

Use of (3.91) and integration by parts shows that the contribution to (3.65) from the term proportional to $A^{2}$ in (3.89) can be written

$$
\begin{aligned}
& -\frac{A}{4} \frac{1}{1-y}\left(\frac{2}{1-x}\right)^{A / 2} \int_{y}^{1} K_{2 n}^{J}(-1, u) d u \\
& +\frac{A}{8} \frac{1}{1-y} \int_{-1}^{1}\left(\frac{1-t}{1-x}\right)^{A \operatorname{sgn}(x-t) / 2}\left(\int_{y}^{1}\left(1-(1-t) \frac{\partial}{\partial t}\right) K_{2 n}^{J}(t, u) d u\right) d t \\
& +\frac{A}{8}\left(\frac{2}{1-x}\right)^{A / 2} \int_{-1}^{1} K(y, t) d t-\frac{A}{8} \int_{-1}^{1}\left(\frac{1-t}{1-x}\right)^{A \operatorname{sgn}(x-t) / 2} K_{2 n}^{J}(y, t) d t .
\end{aligned}
$$

Adding together (3.90), (3.92) and (3.93), and making use of (3.69) gives (3.80), but with the term

$$
\frac{1}{2}(1-x) K_{2 n}^{J}(x, y)
$$

replaced by

$$
\left(\frac{2}{1-x}\right)^{A / 2} K_{2 n}^{J}(-1, y)+\frac{1}{2} \int_{-1}^{x}\left(1-(1-t) \frac{\partial}{\partial t}\right)\left(\frac{1-t}{1-x}\right)^{A / 2} K_{2 n}^{J}(t, y) d t .
$$

In fact (3.94) and (3.95) are equal, as is deduced by integration by parts in the latter. 
In the orthogonal symmetry limit, $A=0$, the results of Proposition 11 can be written

$$
\begin{array}{rlr}
f^{22}(x, y) & = \begin{cases}\frac{1}{2} K_{2 n}^{L}(x, y)-\frac{1}{2} \frac{\partial}{\partial y} \int_{0}^{x} K_{2 n}^{L}(t, y) d t, & \text { Laguerre } \\
\frac{1}{2}(1-x) K_{2 n}^{J}(x, y)-\frac{1}{2}\left((1-y) \frac{\partial}{\partial y}-1\right) \int_{-1}^{x} K_{2 n}^{J}(t, y) d t, & \text { Jacobi }\end{cases} \\
2 f^{12}(x, y) & =\frac{\partial}{\partial x} f^{22}(x, y) & \\
\frac{1}{2} f^{21}(x, y) & =-\frac{1}{2} \operatorname{sgn}(x-y)-\int_{x}^{y} f^{22}(x, t) d t .
\end{array}
$$

The form of $f^{22}(x, y)$ given here is different to the form known from earlier literature (see Section 5.1).

It is also of interest to specialize Proposition 11 in the symplectic symmetry limit, $A \rightarrow-\infty$. Integration by parts shows

$$
\begin{aligned}
f^{22}(x, y) & \sim \begin{cases}\frac{1}{2} K_{2 n}^{L}(x, y)+\frac{1}{2} \frac{\partial}{\partial x} \int_{y}^{\infty} K_{2 n}^{L}(t, x) d t, & \text { Laguerre } \\
\frac{1}{2}(1-x) K_{2 n}^{J}(x, y)+\frac{1}{2} \frac{1-x}{1-y}\left((1-x) \frac{\partial}{\partial x}-1\right) \int_{y}^{1} K_{2 n}^{J}(t, x) d t, & \text { Jacobi }\end{cases} \\
\frac{8}{A^{2}} f^{12}(x, y) & \sim \begin{cases}-\int_{y}^{x} f^{22}(t, y) d t, & \text { Laguerre } \\
-\frac{1}{1-x} \int_{y}^{x} \frac{f^{22}(t, y)}{(1-t)} d t, & \text { Jacobi }\end{cases} \\
\frac{A^{2}}{8} f^{21}(x, y) & \sim \begin{cases}\frac{\partial}{\partial y} f^{22}(x, y), & \text { Laguerre } \\
(1-y) \frac{\partial}{\partial y}(1-y) f^{22}(x, y), & \text { Jacobi }\end{cases}
\end{aligned}
$$

Equivalently, these formulas can be written

$$
\begin{aligned}
\frac{A^{2}}{8} f^{21}(x, y) & \sim \begin{cases}\frac{1}{2}\left(\int_{x}^{\infty} K_{2 n}^{L}(y, t) d t-\int_{y}^{\infty} K_{2 n}^{L}(x, t) d t\right), & \text { Laguerre } \\
\frac{1}{2}\left(\frac{1}{1-x} \int_{x}^{1} K_{2 n}^{J}(y, t) d t-\frac{1}{1-y} \int_{y}^{1} K_{2 n}^{J}(x, t) d t\right), & \text { Jacobi }\end{cases} \\
f^{22}(x, y) & \sim \begin{cases}\frac{\partial}{\partial x} \frac{A^{2}}{8} f^{21}(y, x), & \text { Laguerre } \\
(1-x) \frac{\partial}{\partial x}(1-x) \frac{A^{2}}{8} f^{21}(y, x), & \text { Jacobi }\end{cases} \\
\frac{8}{A^{2}} f^{12}(x, y) & \sim \begin{cases}-\frac{\partial}{\partial y \partial x} \frac{A^{2}}{8} f^{21}(x, y), & \text { Laguerre } \\
-(1-x)(1-y) \frac{\partial^{2}}{\partial x \partial y}(1-x)(1-y) \frac{A^{2}}{8} f^{21}(y, x), & \text { Jacobi }\end{cases}
\end{aligned}
$$

Defining

$$
\tilde{f}^{21}(x, y)= \begin{cases}\lim _{A \rightarrow-\infty} \frac{A^{2}}{8} f^{21}(x, y), & \text { Laguerre } \\ (1-x)(1-y) \lim _{A \rightarrow-\infty} \frac{A^{2}}{8} f^{21}(x, y), & \text { Jacobi }\end{cases}
$$

it follows from (3.102)-(3.104), the first equality in 3.65$)$ and the quaternion determinant formula (1.31), that the $k$-point correlation is identical in structure to (3.64), but with $f^{12}$ replaced by $\tilde{f}^{21}$. Furthermore, comparing (3.102) and (3.105) with (3.59), (3.60) we see that

$$
\tilde{f}^{21}(x, y)=2 f_{\mathrm{ee}}^{12}(x, y) .
$$


This is consistent with the fact that both the even-even correlation (3.64) and the $A \rightarrow-\infty$ limit of the parity blind correlations coincide with the correlations for the corresponding symplectic ensemble (the factor of 2 in (3.106) is due to the double degeneracy inherent in the $A \rightarrow-\infty$ limit).

\subsection{Summation formulas - the odd-even and even-odd blocks}

The blocks $f_{\text {eo }}$ and $f_{\mathrm{oe}}$ in (3.7) are duals in the sense of (3.2), and thus

$$
f_{\mathrm{eo}}(y, x)=\left[\begin{array}{cc}
f_{\mathrm{oe}}^{22}(x, y) & -f_{\mathrm{oe}}^{12}(x, y) \\
-f_{\mathrm{oe}}^{21}(x, y) & f_{\mathrm{oe}}^{11}(x, y)
\end{array}\right] .
$$

Consequently, it suffices to consider one of these blocks, $f_{\text {oe }}$ say. Now, analogous to the formulas (3.65)(3.67) expressing the elements of the matrix $f(x, y)$ in terms of $f_{\mathrm{ee}}^{21}$, we can express the elements of $f_{\mathrm{oe}}(x, y)$ in terms of $f_{\mathrm{ee}}^{21}$. Thus we see from (3.7) that

$$
\begin{aligned}
f_{\mathrm{oe}}^{11}(x, y) & =f_{\mathrm{ee}}^{21}(x, y) \\
f_{\mathrm{oe}}^{12}(x, y) & =-\int_{y}^{\infty} \kappa(t, y) f_{\mathrm{ee}}^{21}(x, t) d t=-f_{\mathrm{ee}}^{11}(y, x) \\
f_{\mathrm{oe}}^{21}(x, y) & =\int_{-\infty}^{x} \kappa(x, t) f_{\mathrm{ee}}^{21}(t, y) d t \\
f_{\mathrm{oe}}^{22}(x, y) & =-\kappa(x, y)-\int_{-\infty}^{x} d t \kappa(x, t) \int_{y}^{\infty} d s \kappa(s, y) f_{\mathrm{ee}}^{21}(t, s) .
\end{aligned}
$$

The last two formulas can be rewritten to read

$$
\begin{aligned}
& f_{\mathrm{oe}}^{21}(x, y)=-f^{22}(x, y)+f_{\mathrm{ee}}^{11}(x, y) \\
& f_{\mathrm{oe}}^{21}(x, y)=-\kappa(x, y)-\int_{y}^{\infty} \kappa(s, y) f^{22}(x, s) d s+f_{\mathrm{ee}}^{12}(x, y) .
\end{aligned}
$$

The only quantity in these formulas which is not known explicitly from our study of the even-even blocks and the parity blind case is the integral in (3.108). Its simplified form is readily computed.

Proposition 12. In the Laguerre case

$$
\begin{aligned}
\int_{y}^{\infty} \kappa(s, y) f^{22}(x, s) d s= & \frac{1}{2} \int_{0}^{x} e^{A(x-t) / 2} K_{2 n}^{L}(t, y) d t+\frac{1}{2} \int_{0}^{x} d t e^{A(x-t) / 2} \int_{y}^{\infty} d u \frac{\partial}{\partial t} K_{2 n}^{L}(u, t) \\
& +\frac{1}{2} e^{A x / 2} \int_{y}^{\infty} K_{2 n}^{L}(0, t) d t
\end{aligned}
$$

while in the Jacobi case

$$
\begin{aligned}
\int_{y}^{\infty} & \kappa(s, y) f^{22}(x, s) d s=\frac{1}{2}(1-y) \int_{-1}^{x}\left(\frac{1-t}{1-x}\right)^{A / 2} K_{2 n}^{J}(t, y) d t \\
& -\frac{1}{2} \int_{-1}^{x} d t\left(\frac{1-t}{1-x}\right)^{A / 2} \int_{y}^{1} d u\left(1-(1-t) \frac{\partial}{\partial t}\right) K_{2 n}^{J}(t, u)+\left(\frac{2}{1-x}\right)^{A / 2} \int_{y}^{1} K_{2 n}^{J}(-1, t) d t(3.1
\end{aligned}
$$

Proof. In the Laguerre case $f^{22}(x, s)$ is given by (3.77), while in the Jacobi case it is given by (3.80). Substituting these formulas as appropriate, and simplifying according to the strategy of the proof of Proposition 11 gives the stated formulas. 


\subsection{Summation formulas — the odd-odd block}

We read off from (3.7) that

$$
\begin{aligned}
f_{\mathrm{oo}}^{11}\left(x, x^{\prime}\right) & =f_{\mathrm{oo}}^{22}\left(x, x^{\prime}\right)=-f_{\mathrm{oe}}^{21}\left(x^{\prime}, x\right) \\
f_{\mathrm{oO}}^{12}\left(x, x^{\prime}\right) & =-f_{\mathrm{ee}}^{22}\left(x, x^{\prime}\right) \\
f_{\mathrm{oO}}^{21}\left(x, x^{\prime}\right) & =\int_{-\infty}^{x} d t \kappa(x, t) \int_{-\infty}^{x^{\prime}} d s \kappa\left(x^{\prime}, s\right) f_{\mathrm{oe}}^{21}(t, s) \\
& =f^{21}\left(x, x^{\prime}\right)+f_{\mathrm{ee}}^{12}\left(x, x^{\prime}\right)-f_{\mathrm{oe}}^{22}\left(x^{\prime}, x\right)-f_{\mathrm{oe}}^{22}\left(x, x^{\prime}\right) .
\end{aligned}
$$

The quantity $f_{\mathrm{oe}}^{21}$ is specified by (3.108) (with $f^{22}$ and $f_{\mathrm{ee}}^{11}$ therein having the explicit forms (3.77), (3.80) and (3.62), (3.63) respectively); $f_{\mathrm{ee}}^{22}$ is given by the explicit forms (3.79), (3.82); $f_{\mathrm{ee}}^{12}$ by (3.59), (3.60); $f_{\mathrm{oe}}^{22}$ by (3.108) (with $f_{\mathrm{ee}}^{12}$ given as noted, and the integral by Proposition 12).

\section{Scaled form of the correlations}

\subsection{Superimposed orthogonal ensembles with a parameter}

Consider first the Laguerre case. To leading order, the support of the spectrum for the LOE is $[0,4 n]$. As has been identified in previous studies (see e.g. [14]), there are three distinct scaling regimes in which different limiting forms of the correlations are obtained. These are the hard edge, specified by the change of scale

$$
x_{j} \mapsto X_{j} / 4 n,
$$

the bulk of the spectrum, specified by

$$
x_{j} \mapsto c+\pi X_{j} / \sqrt{n}, \quad 0<c<4 n \text { (fixed) }
$$

and the soft edge, specified by

$$
x_{j} \mapsto 4 n+2(2 n)^{1 / 3} X_{j} .
$$

In general, under the linear change of scale

$$
x_{j}=a(n)+b(n) X_{j},
$$

the correlation functions transform to correlation functions in the new variables $\left\{X_{j}\right\}$ according to

$$
\rho_{k}\left(X_{1}, \ldots, X_{k}\right)=(b(n))^{k} \rho_{k}\left(x_{1}, \ldots, x_{k}\right) .
$$

The significance of the particular scales (4.1)-(4.3) is that

$$
\rho_{k}^{\text {scaled }}\left(X_{1}, \ldots, X_{k}\right):=\lim _{n \rightarrow \infty}(b(n))^{k} \rho_{k}\left(a(n)+b(n) X_{1}, \ldots, a(n)+b(n) X_{k}\right)
$$

is well defined.

For the parameter dependent extension of the LOE (1.4), and the parameter dependent extension of the superimposed ensemble LOE $\cup \mathrm{LOE}$ (1.8), we expect the hard edge, bulk and soft edge scaled limits to again all be well defined provided the scale of the parameter $A$ is suitably chosen. The correct choice can be anticipated by the requirement that the quantity $e^{A(x-y) / 2}$ occurring in the formulas (2.15) and (3.77) - 3.79) be of order unity in the scaled limit. This is achieved by

$$
A \mapsto \begin{cases}4 n \alpha, & \text { hard edge } \\ \sqrt{n} \alpha / \pi, & \text { bulk } \\ \alpha / 2(2 n)^{1 / 3}, & \text { soft edge }\end{cases}
$$


where $\alpha$ denotes the scaled parameter.

Let $c, \omega>0$ be otherwise arbitrary fixed real numbers. Using the asymptotic formulas [37]

$$
\begin{gathered}
e^{-x / 2} x^{a / 2} L_{n}^{a}(x)=n^{\prime-a / 2} \frac{(n+a) !}{n !} J_{a}\left(2\left(n^{\prime} x\right)^{1 / 2}\right)+R_{1}, \\
n^{\prime}=n+(a+1) / 2, \quad R_{1}= \begin{cases}x^{5 / 4} \mathrm{O}\left(n^{a / 2-3 / 4}\right), & c n^{-1} \leq x \leq \omega \\
x^{a / 2+2} \mathrm{O}\left(n^{a}\right), & 0<x \leq c n^{-1}\end{cases}
\end{gathered}
$$

where $J_{a}(z)$ denotes the Bessel function of order $a$,

$$
e^{-x / 2} x^{a / 2} L_{n}^{a}(x)=n^{a / 2} \frac{1}{\pi^{1 / 2}(n x)^{1 / 4}}\left(\cos \left(2(n x)^{1 / 2}-a \pi / 2-\pi / 4\right)+(n x)^{-1 / 2} \mathrm{O}(1)\right), \quad c n^{-1} \leq x \leq \omega
$$

and [35]

$$
e^{-x / 2} x^{a / 2} L_{n}^{a}(x)=n^{a / 2}\left(\frac{(-1)^{n}}{2^{a}(2 n)^{1 / 3}} \mathrm{Ai}(t)+\mathrm{O}\left(e^{-t}\right) \mathrm{o}\left(n^{-1 / 3}\right)\right)
$$

where $x=4 n+2+2(2 n)^{1 / 3} t, t \in\left[t_{0}, \infty\right)$, it is straightforward to derive the well known formulas

$$
\begin{aligned}
K^{\text {hard }}(X, Y) & :=\lim _{n \rightarrow \infty} \frac{1}{4 n} K^{L}\left(\frac{X}{4 n}, \frac{Y}{4 n}\right) \\
& =\left.\chi_{X, Y>0} \frac{J_{a}\left(X^{1 / 2}\right) Y^{1 / 2} J_{a}^{\prime}\left(Y^{1 / 2}\right)-X^{1 / 2} J_{a}^{\prime}\left(X^{1 / 2}\right) J_{a}\left(Y^{1 / 2}\right)}{2(X-Y)}\right|_{a=0} \\
& =\left.\chi_{X, Y>0} \frac{1}{4} \int_{0}^{1} J_{a}(\sqrt{X t}) J_{a}(\sqrt{Y t}) d t\right|_{a=0}, \\
K^{\text {bulk }}(X, Y) & :=\lim _{n \rightarrow \infty} \frac{\pi}{\sqrt{n}} K^{L}\left(c+\frac{\pi X}{\sqrt{n}}, c+\frac{\pi Y}{\sqrt{n}}\right) \\
& =\frac{\sin \pi(X-Y)}{\pi(X-Y)}=\int_{0}^{1} \cos \pi(X-Y) t d t \\
K^{\text {soft }}(X, Y) & :=\lim _{n \rightarrow \infty} 2(2 n)^{1 / 3} K^{L}\left(4 n+2(2 n)^{1 / 3} X, 4 n+2(2 n)^{1 / 3} Y\right) \\
& =\frac{\operatorname{Ai}(X) \operatorname{Ai}^{\prime}(Y)-\operatorname{Ai}(Y) \operatorname{Ai}^{\prime}(X)}{X-Y}=\int_{0}^{\infty} \operatorname{Ai}(X+t) \operatorname{Ai}(Y+t) d t .
\end{aligned}
$$

We see from (2.15) that knowledge of (4.10)-(4.12) immediately gives the scaled form of $K_{\mathrm{ee}}^{L}\left(y, y^{\prime}\right)$, since we have

$$
K_{\mathrm{ee}}^{\text {scaled }}\left(Y, Y^{\prime}\right):=\lim _{n \rightarrow \infty} b(n) K_{n}^{L}\left(y, y^{\prime}\right)=K^{\text {scaled }}\left(Y, Y^{\prime}\right) .
$$

The scaled form of the remaining quantities in $(2.15)$ is also obtained by formally replacing $K_{n}^{L}$ by $K^{\text {scaled }}$. We will see that the derivation is straightforward in the cases of the hard edge and bulk limits. For the soft edge limit the integrations in the formulas for $K_{\mathrm{eo}}^{L}$ and $K_{\mathrm{oo}}^{L}$ are to leading order over the interval $[0,4 n]$ whereas (4.9) applies to the interval $[4 n, \infty)$. To overcome this difficulty the following identity will be used.

Lemma 13. We have

$$
\begin{aligned}
e^{A x / 2} \int_{0}^{x} e^{-A u / 2} K_{n}^{L}(y, u) d u= & \left(\frac{A-1}{A+1}\right)^{n-1} e^{A(x-y) / 2} \int_{y}^{\infty} e^{-(1-A) u / 2}\left(\frac{d}{d u} L_{n}(u)\right) d u \\
& -e^{A x / 2} \int_{x}^{\infty} e^{-A u / 2} K_{n}^{L}(y, u) d u .
\end{aligned}
$$


Proof. It follows from the integration formula 23]

$$
\int_{0}^{\infty} e^{-(1+A) t / 2} L_{k}(t) d t=\frac{2}{1+A}\left(\frac{A-1}{A+1}\right)^{k}
$$

that

$$
\int_{0}^{\infty} e^{-A u / 2} K_{n}^{L}(y, u) d u=\frac{2}{1+A} e^{-y / 2} \sum_{l=0}^{n-1}\left(\frac{A-1}{A+1}\right)^{l} L_{l}(y) .
$$

But the sum in (4.15) can, according to (3.20), be written as an integral involving a single Laguerre polynomial, and (4.14) results.

Proposition 14. Let the term "scaled" refer to any of the hard edge, bulk or soft edge limits as specified by (4.1)-(4.1). Then in addition to (4.13) we have

$$
\begin{aligned}
K_{\mathrm{eo}}^{\text {scaled }}(Y, X) & :=\lim _{n \rightarrow \infty} K_{\mathrm{eo}}^{L}(y, x)=-e^{\alpha(X-Y) / 2} \chi_{x>y}+e^{\alpha X / 2} \int_{-\infty}^{X} e^{-\alpha v / 2} K^{\text {scaled }}(v, Y) d v \\
K_{\mathrm{oe}}^{\text {scaled }}(X, Y) & :=\lim _{n \rightarrow \infty}(b(n))^{2} K_{\mathrm{oe}}^{L}(x, y)=-e^{-\alpha X / 2} \frac{\partial}{\partial X}\left\{e^{\alpha X / 2} K^{\text {scaled }}(X, Y)\right\} \\
K_{\mathrm{oo}}^{\text {scaled }}\left(X, X^{\prime}\right) & :=\lim _{n \rightarrow \infty} b(n) K_{\mathrm{oo}}^{L}\left(x, x^{\prime}\right)=-e^{\alpha\left(X-X^{\prime}\right) / 2} \frac{\partial}{\partial X}\left\{e^{\alpha X / 2} \int_{-\infty}^{X^{\prime}} e^{-\alpha v / 2} K^{\text {scaled }}(X, v) d v\right\},
\end{aligned}
$$

valid for $\alpha \leq 0$ in the hard edge and bulk cases, and for all $\alpha$ in the soft edge case.

Proof. Using the differentiation formula

$$
\frac{d}{d t} L_{p}^{a}(t)=-L_{p-1}^{a+1}(t)
$$

one can check the well known fact that the asymptotic formulas $(4.7)-(4.9)$ remain valid after differentiation. This, together with (4.13), then implies (4.17). Consider next $K_{\mathrm{eo}}^{\text {hard }}$. The explicit form of the remainder term in (4.7) implies it does not contribute to the scaled limit (4.1), rather the sole contribution comes from (4.13), and this implies (4.16) in the hard edge case (note that the lower terminal in (4.16) can be replaced by 0 in this case). For the bulk limit we note from (4.8) and (4.9) that we have

$$
K_{\mathrm{eo}}^{\mathrm{bulk}}(Y, X)=-e^{\alpha(X-Y) / 2} \chi_{x>y}+e^{\alpha X / 2} \int_{-\infty}^{X} e^{-\alpha v / 2} K^{\mathrm{scaled}}(v, Y) d v+R,
$$

where

$$
R=\lim _{\epsilon \rightarrow 0} \lim _{n \rightarrow \infty} e^{A \sqrt{n} c / \pi} e^{\alpha X / 2} \frac{\pi}{\sqrt{n}} \int_{0}^{\epsilon} e^{-A \sqrt{n} v / 2} K_{n}^{L}(v, c) d v,
$$

and in which we are free to choose $\epsilon<c$. Now it follows from (4.7) that

$$
\left|K_{n}^{L}(v, c)\right| \leq \sqrt{n} f(v), \quad 0 \leq v \leq \epsilon
$$

where $f(v)$ is integrable. Thus with $A \leq 0$ the remainder term $R$ in (4.19) vanishes.

The remaining case is the soft edge limit. We make use of the formula (4.14) and the asymptotic expansion (4.9), which together imply

$$
\begin{aligned}
\lim _{\substack{n \rightarrow \infty \\
\text { soft edge }}} b(n) e^{\alpha x / 2} \int_{0}^{x} e^{-\alpha v / 2} K_{n}^{L}(v, y) d y \\
\quad=e^{-\alpha^{3} / 24} e^{\alpha(X-Y) / 2} \int_{Y}^{\infty} e^{\alpha u / 2} \operatorname{Ai}(u) d u-e^{\alpha X / 2} \int_{X}^{\infty} e^{-\alpha u / 2} K^{\text {soft }}(u, Y) d u .
\end{aligned}
$$


The form (4.16) now follows after making use of the easily verified integration formula

$$
\int_{-\infty}^{\infty} e^{\epsilon t} \operatorname{Ai}(t) d t=e^{\epsilon^{3} / 3}
$$

and the integral form of $K^{\text {soft }}$ in (4.12) to deduce that

$$
\int_{-\infty}^{\infty} e^{-\alpha u / 2} K^{\text {soft }}(u, Y) d u=e^{-\alpha^{3} / 24} e^{-\alpha Y / 2} \int_{Y}^{\infty} \operatorname{Ai}(t) e^{\alpha t / 2} d t .
$$

Finally, the result (4.18) follows from the fact, already noted, that the key asymptotic formulas (4.7) -4.9) remain valid under differentiation, together with the method just used to derive (4.16).

We now turn our attention to the Jacobi case. Here there is a hard edge scaling limit in the neighbourhood of both $x=-1$ and $x=1$, as well as a bulk limit. From previous studies (see e.g. [33]) we know the appropriate scales are

$$
x \mapsto 1-\frac{X}{2 n^{2}}, \quad x \mapsto-1+\frac{X}{2 n^{2}}
$$

for the hard edge at $x=1, x=-1$ respectively, and

$$
x \mapsto \cos \theta_{0}-\frac{X}{n} \sin \theta_{0} \sim \cos \left(\theta_{0}+\frac{X}{n}\right), \quad 0<\theta_{0}<\pi
$$

in the bulk. For the scaling of the parameter $A$ we choose

$$
A \mapsto \begin{cases}4 n^{2} \alpha, & \text { hard edge at } x=1 \\ \alpha, & \text { hard edge at } x=-1 \\ n \alpha\left(1-\cos \theta_{0}\right) / \sin \theta_{0}, & \text { bulk }\end{cases}
$$

This is suggested by the criterion that the term $((1-x) /(1-y))^{-A / 2}$ tend to a non-constant order one quantity.

A rigorous analysis of the scaling limits for the JOE and JSE has recently been undertaken by Dueñez [12]. Following the methodology therein, we make use of the asymptotic formulas

$$
\begin{aligned}
& P_{n}^{(a, b)}(\cos \theta)=(\pi n)^{-1 / 2}\left(\sin \frac{\theta}{2}\right)^{-a-1 / 2}\left(\cos \frac{\theta}{2}\right)^{-b-1 / 2} \cos \left(n^{\prime} \theta+\gamma\right)+E_{1}, \\
& n^{\prime}=n+(a+b+1) / 2, \quad \gamma=-\frac{\pi}{2}(a+1 / 2)
\end{aligned}
$$

for $0<\theta<\pi$, where

$$
E_{1}=\theta^{-a-3 / 2} O\left(n^{-3 / 2}\right), \quad \text { uniformly for } c / n \leq \theta \leq \pi-\epsilon(c, \epsilon \ll 1)
$$

and

$$
\left(\sin \frac{\theta}{2}\right)^{a}\left(\cos \frac{\theta}{2}\right)^{b} P_{n}^{(a, b)}(\cos \theta)=n^{-a} \frac{\Gamma(n+a+1)}{n !} \sqrt{\frac{\theta}{\sin \theta}} J_{a}\left(n^{\prime} \theta\right)+E_{2}
$$

for $0 \leq \theta<\pi$, where

$$
E_{2}= \begin{cases}\theta^{1 / 2} O\left(n^{-3 / 2}\right), & c / n \leq \theta \leq \pi-\epsilon \\ \theta^{a+2} O\left(n^{a}\right), & 0<\theta \leq c / n,\end{cases}
$$

again uniformly in $\theta$. The asymptotic form (4.24) is dominant in the bulk, while (4.25) is dominant for the hard edge at $x=1$. To study the hard edge at $x=-1$, we make use of the fact that

$$
P_{n}^{(a, b)}(-\cos \theta)=P_{n}^{(b, a)}(\cos \theta),
$$

and then use (4.25). The strategy of the proof of Proposition 14 then yields the following forms for the matrix elements determining the scaled correlations. 
Proposition 15. The results of Proposition 14 hold for the scaled limit of the matrix elements (2.10) in the bulk and for the hard edge at $x=-1$. For the hard edge at $x=1$ we have

$$
\begin{aligned}
K_{\mathrm{ee}}^{\mathrm{hard}}\left(Y, Y^{\prime}\right) & =\left(\frac{Y}{Y^{\prime}}\right)^{1 / 2} K^{\mathrm{hard}}\left(Y, Y^{\prime}\right) \\
K_{\mathrm{eo}}^{\mathrm{hard}}(Y, X) & =-\left(\frac{X}{Y}\right)^{-\alpha / 2} \chi_{x>y}+\frac{Y^{1 / 2}}{X^{\alpha / 2}} \int_{X}^{\infty} v^{(\alpha-1) / 2} K^{\mathrm{hard}}(v, Y) d v \\
K_{\mathrm{oe}}^{\mathrm{hard}}(X, Y) & =\frac{X^{\alpha / 2}}{Y^{1 / 2}} \frac{\partial}{\partial X}\left\{X^{(1-\alpha) / 2} K^{\mathrm{hard}}(X, Y)\right\} \\
K_{\mathrm{oo}}^{\mathrm{hard}}\left(X, X^{\prime}\right) & =\left(\frac{X}{X^{\prime}}\right)^{\alpha / 2} \frac{\partial}{\partial X}\left\{X^{(1-\alpha) / 2} \int_{X^{\prime}}^{\infty} v^{(\alpha-1) / 2} K^{\mathrm{hard}}(X, v) d v\right\},
\end{aligned}
$$

valid for parameter values $\alpha<3 / 2$. Here $K^{\text {hard }}$ refers to (4.10), but without the restriction $a=0$.

\subsection{Decimated orthogonal ensembles with a parameter}

The scaling limits introduced in the previous subsection in relation to the superimposed orthogonal ensembles with a parameter all carry over to the decimated orthogonal ensembles with a parameter. Furthermore, the strategies used to derive the scaled limits in Propositions 14 and 15 again suffice to derive the scaled limits of the matrix elements determining the correlations for the decimated Laguerre and Jacobi orthogonal ensembles with a parameter. In the interest of economy of space we will restrict ourselves to presenting the explicit form of the scaling limit for the parity blind correlations only.

Proposition 16. Let the term "scaled" refer to any of the hard edge, bulk or soft edge limits for the parameter dependent Laguerre ensembles as specified by (4.1)-(4.0). Then the scaled form of the matrix elements (3.77)-(3.7.) have the explicit form

$$
\begin{aligned}
& f_{\text {scaled }}^{22}(X, Y):=\left.\lim _{n \rightarrow \infty} b(n) f^{22}(x, y)\right|_{2 n \mapsto n}=\frac{1}{2} K^{\text {scaled }}(X, Y) \\
&- \frac{1}{2} \frac{\partial}{\partial Y} \int_{-\infty}^{X} e^{\alpha(X-t) / 2} K^{\text {scaled }}(t, Y) d t-\frac{\alpha}{4} \int_{-\infty}^{X} d t e^{\alpha(X-t) / 2} \int_{Y}^{\infty} d u \frac{\partial}{\partial t} K^{\text {scaled }}(u, t) \\
& f_{\text {scaled }}^{12}(X, Y):=\left.\lim _{n \rightarrow \infty}(b(n))^{2} f^{12}(x, y)\right|_{2 n \mapsto n} \\
&= \frac{1}{4}\left(\frac{\alpha}{2}+\frac{\partial}{\partial X}\right)\left(\frac{\alpha}{2}+\frac{\partial}{\partial Y}\right)\left\{\int_{X}^{\infty} K^{\text {scaled }}(Y, t) d t-\int_{Y}^{\infty} K^{\text {scaled }}(X, t) d t\right\} \\
& f_{\text {scaled }}^{21}(X, Y)=\left.\lim _{n \rightarrow \infty} f^{21}(x, y)\right|_{2 n \mapsto n}=-e^{\alpha|X-Y| / 2} \operatorname{sgn}(X-Y) \\
&-\left\{\int_{-\infty}^{Y} e^{\alpha(Y-t) / 2} K^{\text {scaled }}(X, t) d t-\int_{-\infty}^{X} e^{\alpha(X-t) / 2} K^{\text {scaled }}(Y, t) d t\right\}
\end{aligned}
$$

Proof. The only new feature of the form of the expressions in (3.77)-3.79) relative to those in (2.15) is the need to analyze

$$
\int_{0}^{\infty} K_{2 n}^{L}(y, u) d u
$$

This is done by noting from (3.71) and (3.72) that

$$
\begin{aligned}
\int_{0}^{\infty} K_{2 n}^{L}(y, u) d u & =\frac{1}{2} \int_{y}^{\infty} e^{-u / 2} L_{2 n}(u) d u-e^{-y / 2} L_{2 n}(y) \\
& =1-\frac{1}{2} \int_{0}^{y} e^{-u / 2} L_{2 n}(u) d u-e^{-y / 2} L_{2 n}(y) .
\end{aligned}
$$

Apart from this, we follow the strategy used to deduce the scaled limits in Proposition 14. 
In the Jacobi case the analogue of (4.30) is afforded by (3.74)-(3.76). Using the resulting identity, and employing the strategies used to derive Propositions 14 and 15 gives the following result.

Proposition 17. The results of Proposition 10 hold for the scaled limit of the matrix elements (3.80)3.89) in the bulk and for the hard edge at $x=-1$. For the hard edge at $x=1$ we have

$$
\begin{aligned}
f_{\text {hard }}^{22}(X, Y)= & \frac{1}{2}\left(\frac{X}{Y}\right)^{1 / 2} K^{\text {hard }}(X, Y)+\frac{1}{2} \frac{\partial}{\partial Y} \frac{Y^{1 / 2}}{X^{\alpha / 2}} \int_{X}^{\infty} t^{(\alpha-1) / 2} K^{\text {hard }}(t, Y) d t \\
& -\frac{\alpha}{4 Y} \int_{X}^{\infty} d t\left(\frac{t}{X}\right)^{\alpha / 2} \int_{0}^{Y} d u u^{-1 / 2} \frac{\partial}{\partial t} t^{1 / 2} K^{\text {hard }}(u, t) \\
f_{\text {hard }}^{12}(X, Y)= & -\frac{1}{4}\left(\frac{\alpha}{2 X}-\frac{\partial}{\partial X}\right)\left(\frac{\alpha}{2 Y}-\frac{\partial}{\partial Y}\right) \\
& \times\left\{Y^{1 / 2} \int_{0}^{X} t^{-1 / 2} K^{\text {hard }}(Y, t) d t-X^{1 / 2} \int_{0}^{Y} t^{-1 / 2} K^{\text {hard }}(X, t) d t\right\} \\
f_{\text {hard }}^{21}(X, Y)= & -\left(\frac{X}{Y}\right)^{-\alpha \operatorname{sgn}(X-Y) / 2} \operatorname{sgn}(X-Y) \\
& +\left\{\frac{X^{1 / 2}}{Y^{\alpha / 2}} \int_{Y}^{\infty} t^{(\alpha-1) / 2} K^{\text {hard }}(X, t) d t-\frac{Y^{1 / 2}}{X^{\alpha / 2}} \int_{X}^{\infty} t^{(\alpha-1) / 2} K^{\text {hard }}(Y, t) d t\right\} .
\end{aligned}
$$

valid for parameter values $\alpha<3 / 2$, and where $K^{\text {hard }}$ refers to (4.10), but without the restriction $a=0$ (both (4.39) and (4.33) have been multiplied by -1 - an operation which leaves qdet $\left[f_{\text {hard }}\right]$ unchanged - so as to formally conserve the relations (3.97) and (3.98)).

\section{Discussion}

\subsection{The orthogonal symmetry limit}

In the orthogonal symmetry limit $A=0$ the matrix elements determining the parity blind correlations are specified by (3.96)-(3.98). We have already remarked that the value of $f^{11}(x, y)$ implied by (3.96) differs in structure to that known from previous literature [40, 17, 1]. To present the latter, which are valid for general $a>-1$ in the LOE and general $a, b>-1$ in the JOE, we introduce generalizations of (1.32), (1.36),

$$
\begin{aligned}
K_{n, a}^{L}(x, y) & :=(x y)^{a / 2} e^{-(x+y) / 2} \sum_{l=0}^{n-1} \frac{1}{h_{l, a}^{L}} L_{l}^{a}(x) L_{l}^{a}(y) \\
K_{n, a, b}^{J}(x, y) & :=((1-x)(1-y))^{(a-1) / 2}((1+x)(1+y))^{b / 2} \sum_{l=0}^{n-1} \frac{1}{h_{l, a, b}^{J}} P_{l}^{(a, b)}(x) P_{l}^{(a, b)}(y) .
\end{aligned}
$$

In (5.1), $L_{l}^{a}$ denotes the Laguerre polynomial of degree $l$ with orthogonality property

$$
\int_{0}^{\infty} t^{a} e^{-t} L_{m}^{a}(t) L_{n}^{a}(t) d t=h_{n, a}^{L} \delta_{m, n}, \quad h_{n, a}^{L}=\frac{\Gamma(a+n+1)}{\Gamma(n+1)},
$$

while in 5.2$), P_{l}^{(a, b)}$ denotes the Jacobi polynomial of degree $l$ with orthogonality property

$$
\begin{gathered}
\int_{-1}^{1}(1-t)^{a}(1+t)^{b} P_{m}^{(a, b)}(t) P_{n}^{(a, b)}(t) d t=h_{n, a, b}^{J} \delta_{m, n} \\
h_{n, a, b}^{J}=\frac{\Gamma(a+1+n) \Gamma(b+1+n) 2^{a+b+1}}{\Gamma(n+1) \Gamma(a+b+1+n)(a+b+1+2 n)} .
\end{gathered}
$$


The case $a=0$ of (5.1) and $b=0$ of (5.2) reduces to (1.32) and 1.36) respectively.

In terms of (5.1) and (5.2), the results of [1] give that the $k$-point distribution for matrix ensembles $\mathrm{OE}_{n}\left(x^{a / 2} e^{-x / 2}\right)(\mathrm{LOE})$ and $\mathrm{OE}_{n}\left((1+x)^{b / 2}(1-x)^{(a-1) / 2}\right)(\mathrm{JOE}), n$ even, as specified by (1.11) are given by (1.31) with the $2 \times 2$ matrix $f$ in (1.31) having as its top left entry

$$
f^{11}(x, y)=\left(\frac{x}{y}\right)^{1 / 2} K_{n-1, a+1}^{L}(x, y)-\frac{1}{4 h_{n-1, a}^{L}} y^{a / 2} e^{-y / 2} L_{n-1}^{a+1}(y) \int_{0}^{\infty} \operatorname{sgn}(x-u) L_{n-2}^{a+1}(u) u^{a / 2} e^{-u / 2} d u
$$

in the Laguerre case, and

$$
\begin{aligned}
f^{11}(x, y)= & (1-x)\left(\frac{1+x}{1+y}\right)^{1 / 2} K_{n-1, a, b+1}^{J}(x, y)+\frac{1}{4 h_{n-1, a, b}^{J}} \frac{(a+b+n)(a-1+n)}{(a+b+2 n-1)}(1-y)^{(a-1) / 2} \\
& \times(1+y)^{b / 2} P_{n-1}^{(a, b+1)}(y) \int_{-1}^{1} \operatorname{sgn}(x-u) P_{n-2}^{(a, b+1)}(u)(1-u)^{(a-1) / 2}(1+u)^{b / 2} d u,
\end{aligned}
$$

in the Jacobi case. The other entries in the matrix $f$ are related to $f^{11}$ by

$$
f^{22}(x, y)=f^{11}(y, x), \quad f^{21}(x, y)=-\frac{1}{2} \operatorname{sgn}(x-y)-\int_{x}^{y} f^{11}(x, u) d u, \quad f^{12}(x, y)=\frac{\partial}{\partial x} f^{11}(x, y),
$$

which are identical to the structure of the formulas obtained in Section 3.4 provided we interchange $f^{11}$ and $f^{22}$ (recall the first equality in (3.65), (3.97), (3.98)).

We know from [40, 17, 1] that (5.4) can be rewritten to read

$$
f^{11}(x, y)=K_{n, a}^{L}(x, y)+F_{1}^{L}(y) F_{2}^{L}(x)
$$

where

$$
\begin{aligned}
F_{1}^{L}(y) & =\frac{1}{4 h_{n-1, a}^{L}} y^{a / 2} e^{-y / 2} L_{n-1}^{a+1}(y) \\
F_{2}^{L}(x) & =\left(-4 x^{a / 2} e^{-x / 2} L_{n-1}^{a}(x)-\int_{0}^{\infty} \operatorname{sgn}(x-u) L_{n-2}^{a+1}(u) u^{a / 2} e^{-u / 2} d u\right) \\
& =-n \int_{0}^{\infty} \operatorname{sgn}(x-t) t^{a / 2-1} e^{-t / 2}\left(L_{n}^{a}(t)-L_{n-1}^{a}(t)\right) d t .
\end{aligned}
$$

The formula (5.5) in the Jacobi case can similarly be rewritten.

Lemma 18. The formula (5.5) has the alternative form

$$
f^{11}(x, y)=(1-x) K_{n, a, b}^{J}(x, y)+F_{1}^{J}(y) F_{2}^{J}(x)
$$

where

$$
\begin{gathered}
F_{1}^{J}(y)=\frac{1}{4 h_{n-1, a, b}^{J}}\left(\frac{a+b+n}{a+b-1+2 n}\right)(1-y)^{(a-1) / 2}(1+y)^{b / 2} P_{n-1}^{(a, b+1)}(y) \\
F_{2}^{J}(x)=\left(-4(1-x)^{(a+1) / 2}(1+x)^{b / 2} P_{n-1}^{(a, b)}(x)\right. \\
\left.+(a-1+n) \int_{-1}^{1} \operatorname{sgn}(x-u) P_{n-2}^{(a, b+1)}(u)(1-u)^{(a-1) / 2}(1+u)^{b / 2} d u\right) \\
=\frac{2 n}{2 n+a+b} \int_{-1}^{1} \operatorname{sgn}(x-u)\left((n+a+b) P_{n}^{(a, b)}(u)+(n+a) P_{n-1}^{(a, b)}(u)\right)(1-u)^{(a-1) / 2}(1+u)^{b / 2-1} d u .
\end{gathered}
$$


Proof. It follows from (5.3) that

$$
\frac{1}{h_{l, a, b+1}^{J}}=\frac{(a+b+1+l)(a+b+2+2 l)}{2(b+1+l)(a+b+1+2 l)} \frac{1}{h_{l, a, b}^{J}} .
$$

Using this, and the Jacobi polynomial identity

$$
\left(l+\frac{a+b}{2}+1\right)(1+x) P_{l}^{(a, b+1)}(x)=(l+b+1) P_{l}^{(a, b+1)}(x)+(l+1) P_{l+1}^{(a, b)}(x),
$$

we see that

$$
\begin{aligned}
(1+ & x) \sum_{l=0}^{n-2} \frac{1}{h_{l, a, b+1}^{J}} P_{l}^{(a, b+1)}(x) P_{l}^{(a, b+1)}(y) \\
& =\sum_{l=0}^{n-1} \frac{1}{h_{l, a, b}^{J}}\left(\frac{1}{a+b+1+2 l}\right) P_{l}^{(a, b)}(x)\left\{(a+b+1+l) P_{l}^{(a, b+1)}(y)+(a+l) P_{l-1}^{(a, b+1)}(y)\right\} \\
& -\frac{1}{h_{n-1, a, b}^{J}}\left(\frac{a+b+n}{a+b-1+2 n}\right) P_{n-1}^{(a, b)}(x) P_{n-1}^{(a, b+1)}(y) .
\end{aligned}
$$

Simplifying the term in the brackets \{\} using the Jacobi polynomial identity

$$
(a+b+1+l) P_{l}^{(a, b+1)}(x)+(a+l) P_{l-1}^{(a, b)}(x)=(a+b+1+2 l) P_{l}^{(a, b)}(x),
$$

recalling the definition (5.2), and substituting the result in (5.5) we deduce (5.10) with $F_{2}^{J}(x)$ given by the first equality in (5.12). The second equality in (5.12) can be deduced from the first by verifying that both expressions agree at $x=1$, and then verifying that the derivative of both expressions agrees.

We now equate the RHSs of the final equation in (5.6), with the substitutions (5.7) and (5.10) (and $n \mapsto 2 n)$, and (3.97), with the substitution (3.96). In the Laguerre case this gives

$$
\frac{1}{2}\left(\frac{\partial}{\partial x}+\frac{\partial}{\partial y}\right) K_{2 n}^{L}(x, y)=c_{2 n, 0}^{L} \phi_{2 n, 0}^{L}(x) \psi_{2 n, 0}^{L}(y)
$$

where

$$
\begin{aligned}
c_{n, a}^{L} & =\frac{n}{2 h_{n-1, a}^{L}} \\
\phi_{n, a}^{L}(x) & =x^{a / 2} e^{-x / 2} L_{n-1}^{a+1}(x)=-x^{a / 2-1} e^{-x / 2}\left(n L_{n}^{a}(x)-(n+a) L_{n-1}^{a}(x)\right) \\
\psi_{n, a}^{L}(y) & =y^{a / 2-1} e^{-y / 2}\left(L_{n}^{a}(y)-L_{n-1}^{a}(y)\right)
\end{aligned}
$$

(the parameter $a$ has been kept general in (5.14) for later convenience). Note from (5.14) that the RHS of (5.13) is symmetric in $x$ and $y$, as is the LHS. The substitutions in the Jacobi case give

$$
\frac{1}{2}\left((1-x) \frac{\partial}{\partial x}+(1-y) \frac{\partial}{\partial y}\right)(1-x)(1-y) K_{2 n}^{J}(x, y)=c_{2 n, a, 0}^{J} \phi_{2 n, a, 0}^{J}(x) \psi_{2 n, a, 0}^{J}(y)
$$

where

$$
\begin{aligned}
c_{n, a, b}^{J} & =\frac{1}{2 h_{n-1, a, b}^{J}}\left(\frac{a+b+n}{a+b-1+2 n}\right)\left(\frac{2 n}{2 n+a+b}\right) \\
\phi_{n, a, b}^{J}(x) & =(1-x)^{(a+1) / 2} P_{n-1}^{(a, b+1)}(x) \\
& =(1-x)^{(a+1) / 2}(1+x)^{b / 2-1} \frac{2}{2 n+a+b}\left((n+b) P_{n-1}^{(a, b)}(x)+n P_{n}^{(a, b)}(x)\right) \\
\psi_{n, a, b}^{J}(y) & =-(1-y)^{(a+1) / 2}(1+y)^{b / 2-1}\left((n+a+b) P_{n}^{(a, b)}(y)+(n+a) P_{n-1}^{(a, b)}(y)\right) .
\end{aligned}
$$


As with (5.13), we see from (5.16) that the RHS of (5.15) is symmetric in $x$ and $y$, as is the LHS.

The identity (5.13) is the special case $a=0$ of the identity [40]

$$
\left(\frac{\partial}{\partial x}+\frac{\partial}{\partial y}\right) K_{n, a}^{L}(x, y)=c_{n, a}^{L}\left(\phi_{n, a}^{L}(x) \psi_{n, a}^{L}(y)+\phi_{n, a}^{L}(y) \psi_{n, a}^{L}(x)\right) .
$$

A simple consequence of (5.17) is the integral representation 29]

$$
K_{n, a}^{L}(x, y)=c_{n, a}^{L} \int_{0}^{\infty}\left(\phi_{n, a}^{L}(x+t) \psi_{n, a}^{L}(y+t)+\phi_{n, a}^{L}(y+t) \psi_{n, a}^{L}(x+t)\right) d t .
$$

The result (5.15) suggests an analogue of (5.17), and (5.18), in the Jacobi case.

Proposition 19. With $K_{n, a, b}^{J}$ specified by (5.9), and $c_{n, a}^{J}, \phi_{n, a, b}^{J}(x), \psi_{n, a, b}^{J}(y)$ by (5.10), we have

$$
\left((1-x) \frac{\partial}{\partial x}+(1-y) \frac{\partial}{\partial y}\right)(1-x)(1-y) K_{n, a, b}^{J}(x, y)=c_{n, a, b}^{J}\left(\phi_{n, a, b}^{J}(x) \psi_{n, a, b}^{J}(y)+\psi_{n, a, b}^{J}(x) \phi_{n, a, b}^{J}(y)\right),
$$

and consequently, for $0<x, y<1$,

$$
4 x y K_{n, a, b}^{J}(1-2 x, 1-2 y)=c_{n, a, b}^{J} \int_{0}^{1}\left(\phi_{n, a, b}^{J}(1-x u) \psi_{n, a, b}^{J}(1-y u)+\phi_{n, a, b}^{J}(1-y u) \psi_{n, a, b}^{J}(1-x u)\right) \frac{d u}{u}
$$

Proof. We generally follow the strategy used in 40 in the Laguerre case. But before doing so we note the general identity

$$
\left((1-x) \frac{\partial}{\partial x}+(1-y) \frac{\partial}{\partial y}\right)\left(\frac{(1-x)^{1 / 2}(1-y)^{1 / 2}}{x-y} f\right)=\frac{(1-x)^{1 / 2}(1-y)^{1 / 2}}{x-y}\left((1-x) \frac{\partial}{\partial x}+(1-y) \frac{\partial}{\partial y}\right) f .
$$

To make use of this identity we note from the Christoffel-Darboux formula (see e.g. [37) that

$$
K_{n, a, b}^{J}(x, y)=((1-x)(1-y))^{(a-1) / 2}((1+x)(1+y))^{b / 2} \bar{a}_{n} \frac{P_{n}^{(a, b)}(x) P_{n-1}^{(a, b)}(y)-P_{n}^{(a, b)}(y) P_{n-1}^{(a, b)}(x)}{x-y}
$$

where

$$
\bar{a}_{n}=\frac{n !}{2^{a+b}} \frac{\Gamma(n+a+b+1)}{\Gamma(n+a) \Gamma(n+b)(2 n+a+b)} .
$$

Thus

$$
\begin{aligned}
& \left((1-x) \frac{\partial}{\partial x}+(1-y) \frac{\partial}{\partial y}\right)(1-x)(1-y) K_{n, a, b}^{J}(x, y) \\
& \quad=\frac{(1-x)^{1 / 2}(1-y)^{1 / 2}}{x-y} \bar{a}_{n}\left((1-x) \frac{\partial}{\partial x}+(1-y) \frac{\partial}{\partial y}\right)\left(q_{n}(x) q_{n-1}(y)-q_{n}(y) q_{n-1}(x)\right)
\end{aligned}
$$

where

$$
q_{n}(x):=(1-x)^{a / 2}(1+x)^{b / 2} P_{n}^{(a, b)}(x),
$$

so the task is to compute the action of the operator on the RHS of (5.21).

For this purpose, we note from suitable Jacobi polynomial formulas that

$$
\begin{aligned}
\left(1-x^{2}\right) q_{n}^{\prime}(x) & =\left(\alpha_{0}+\alpha_{1} x\right) q_{n}(x)+\beta_{0} q_{n-1}(x) \\
\left(1-x^{2}\right) q_{n-1}^{\prime}(x) & =-\gamma_{0} q_{n}(x)-\left(\alpha_{0}+\alpha_{1} x\right) q_{n-1}(x)
\end{aligned}
$$

with

$$
\begin{aligned}
& \alpha_{0}+\alpha_{1} x=\frac{b^{2}-a^{2}}{2(2 n+a+b)}-\frac{(2 n+a+b)}{2} x \\
& \beta_{0}=\frac{2(n+a)(n+b)}{2 n+a+b}, \quad \gamma_{0}=\frac{2 n(n+a+b)}{2 n+a+b} .
\end{aligned}
$$


Equivalently

$$
\left[\begin{array}{c}
(1-x) q_{n}^{\prime}(x) \\
(1-x) q_{n-1}^{\prime}(x)
\end{array}\right]=\left[\begin{array}{cc}
A(x) & B(x) \\
-C(x) & -A(x)
\end{array}\right]\left[\begin{array}{c}
q_{n}(x) \\
q_{n-1}(x)
\end{array}\right]
$$

where

$$
A(x)=\frac{\alpha_{0}+\alpha_{1} x}{1+x}, \quad B(x)=\frac{\beta_{0}}{1+x}, \quad C(x)=\frac{\gamma_{0}}{1+x} .
$$

Introducing the matrix formulation

$$
q_{n}(x) q_{n-1}(y)-q_{n}(y) q_{n-1}(x)=\left[\begin{array}{ll}
q_{n}(x) & q_{n-1}(x)
\end{array}\right]\left[\begin{array}{cc}
0 & 1 \\
-1 & 0
\end{array}\right]\left[\begin{array}{c}
q_{n}(y) \\
q_{n-1}(y)
\end{array}\right],
$$

a straightforward calculation using (5.23) shows

$$
\begin{aligned}
& \frac{1}{x-y}\left((1-x) \frac{\partial}{\partial x}+(1-y) \frac{\partial}{\partial y}\right)\left(q_{n}(x) q_{n-1}(y)-q_{n}(y) q_{n-1}(x)\right) \\
& =\left[\begin{array}{ll}
q_{n}(x) & q_{n-1}(x)
\end{array}\right]\left[\begin{array}{ll}
\frac{C(x)-C(y)}{x-y} & \frac{A(x)-A(y)}{x-y} \\
\frac{A(x)-A(y)}{x-y} & \frac{B(x)-B(y)}{x-y}
\end{array}\right]\left[\begin{array}{c}
q_{n}(y) \\
q_{n-1}(y)
\end{array}\right] \\
& =-\frac{1}{(1+x)(1+y)}\left[\begin{array}{ll}
q_{n}(x) & q_{n-1}(x)
\end{array}\right]\left[\begin{array}{cc}
\gamma_{0} & \alpha_{0}-\alpha_{1} \\
\alpha_{0}-\alpha_{1} & \beta_{0}
\end{array}\right]\left[\begin{array}{c}
q_{n}(y) \\
q_{n-1}(y)
\end{array}\right] \\
& =-\frac{1}{(1+x)(1+y)}(1-x)^{a / 2}(1+x)^{b / 2}(1-y)^{a / 2}(1+y)^{b / 2} \frac{2}{2 n+a+b} \\
& \times\left[n(n+a+b) P_{n}^{(a, b)}(x) P_{n}^{(a, b)}(y)+(n+a)(n+b) P_{n-1}^{(a, b)}(x) P_{n-1}^{(a, b)}(y)\right. \\
& \left.+\frac{1}{4}\left(b^{2}-a^{2}+(2 n+a+b)^{2}\right)\left(P_{n}^{(a, b)}(x) P_{n-1}^{(a, b)}(y)+P_{n}^{(a, b)}(y) P_{n-1}^{(a, b)}(x)\right)\right]
\end{aligned}
$$

Substituting (5.24) in (5.21) gives (5.19).

With (5.19) established, we make the changes of variables

$$
x=1-2 e^{-s}, \quad y=1-2 e^{-t}, \quad 0 \leq s, t<\infty
$$

which gives

$$
\begin{aligned}
& \left(\frac{\partial}{\partial s}+\frac{\partial}{\partial t}\right) 4 e^{-(s+t)} K_{n, a, b}^{J}\left(1-2 e^{-s}, 1-2 e^{-t}\right) \\
& \quad=c_{n, a, b}^{J}\left(\phi_{n, a, b}^{J}\left(1-2 e^{-s}\right) \psi_{n, a, b}^{J}\left(1-2 e^{-t}\right)+\phi_{n, a, b}^{J}\left(1-2 e^{-t}\right) \psi_{n, a, b}^{J}\left(1-2 e^{-s}\right)\right) .
\end{aligned}
$$

A simple calculation using integration by parts verifies that

$$
\begin{aligned}
& 4 e^{-(s+t)} K_{n, a, b}^{J}\left(1-2 e^{-s}, 1-2 e^{-t}\right)=c_{n, a, b}^{J} \\
& \quad \times \int_{0}^{\infty}\left(\phi_{n, a, b}^{J}\left(1-2 e^{-s-z}\right) \psi_{n, a, b}^{J}\left(1-2 e^{-t-z}\right)+\phi_{n, a, b}^{J}\left(1-2 e^{-t-z}\right) \psi_{n, a, b}^{J}\left(1-2 e^{-s-z}\right)\right) d z .(5.2
\end{aligned}
$$

satisfies (5.25). The general solution of (5.25) is (5.26) plus an arbitrary function of $s-t$. But we see from the definition of $K_{n, a, b}^{J}, \phi_{n, a, b}^{J}$ and $\psi_{n, a, b}^{J}$ that both sides of (5.26) vanish in the limits $s, t \rightarrow \infty$, $s-t$ fixed, so this arbitrary function must be chosen to be the zero function. Putting $e^{-s}=x, e^{-t}=y$ in (5.26) and changing variables $e^{-z}=u$ gives (5.20).

The orthogonal symmetry limit of the scaled matrix elements of Propositions 16 and 17 is obtained by setting $\alpha=0$. We see from the results of Propositions 16 and 17 (with $f^{11}$ and $f^{22}$ interchanged for 
convenience) that the $k$-point distributions are then given by (1.31) with the $2 \times 2$ matrix $f$ having as its top left entry

$$
f_{\text {scaled }}^{11}(X, Y)=\frac{1}{2} K^{\text {scaled }}(X, Y)-\frac{1}{2} \frac{\partial}{\partial Y} \int_{-\infty}^{X} K^{\text {scaled }}(t, Y) d t
$$

in the case of the soft edge and bulk ( $K^{\text {scaled }}$ as given by (4.12) and (4.11) respectively), and

$$
f_{\text {scaled }}^{11}(X, Y)=\frac{1}{2}\left(\frac{X}{Y}\right)^{1 / 2} K^{\text {hard }}(X, Y)+\frac{1}{2} \frac{\partial}{\partial Y} Y^{1 / 2} \int_{X}^{\infty} t^{-1 / 2} K^{\text {hard }}(t, Y) d t
$$

in the case of the hard edge with singularity $x^{(a-1) / 2}$ as $x \rightarrow 0$ ( $K^{\text {hard }}$ is given by 4.10 ), without the restriction $a=0)$. The remaining entries in $f$ are related to $f^{11}$ as in (5.6).

The formula (5.28) has been read off from Proposition 17, deduced from the scaled limit of the Jacobi case. The hard edge singularity $\left.x^{(a-1) / 2}\right|_{a=1}$ as $x \rightarrow 0$ is also contained in the results of Proposition 16 for the scaled Laguerre case. In this case we read off from Proposition 16 the alternative formula

$$
f_{\text {scaled }}^{11}(X, Y)=\left.\frac{1}{2} K^{\text {hard }}(X, Y)\right|_{a=0}-\left.\frac{1}{2} \frac{\partial}{\partial Y} \int_{0}^{X} K^{\text {hard }}(t, Y)\right|_{a=0} d t,
$$

which differs in form to (5.28) with $a=1$. Our first discussion point regarding the form of $f_{\text {scaled }}^{11}$ exhibited by (5.27) and (5.28) is to reconcile the two seemingly different expressions in the case of the hard edge singularity $\left.x^{(a-1) / 2}\right|_{a=1}$ as $x \rightarrow 0$. For this purpose we make use of the following recurrence.

Lemma 20. Let $K^{\text {hard }}(X, Y)$ be given by $(4.10)$, without the restriction to a $=0$. We have

$$
\left.\left(\frac{X}{Y}\right)^{1 / 2} K^{\mathrm{hard}}(X, Y)\right|_{a \rightarrow a+1}=K^{\mathrm{hard}}(X, Y)-\frac{1}{2} Y^{-1 / 2} J_{a}\left(X^{1 / 2}\right) J_{a+1}\left(Y^{1 / 2}\right)
$$

where it is understood that $X, Y>0$.

Proof. We first make use of the Bessel function identity

$$
u J_{a}^{\prime}(u)=a J_{a}(u)-u J_{a+1}(u)
$$

to rewrite the first equality in (4.10), without the restriction to $a=0$, as

$$
K^{\mathrm{hard}}(X, Y)=\frac{X^{1 / 2} J_{a+1}\left(X^{1 / 2}\right) J_{a}\left(Y^{1 / 2}\right)-Y^{1 / 2} J_{a+1}\left(Y^{1 / 2}\right) J_{a}\left(X^{1 / 2}\right)}{2(X-Y)} .
$$

The stated formula now follows by replacing $a$ by $a+1$, using the Bessel function identity

$$
u J_{a+2}(u)=2(a+1) J_{a+1}(u)-u J_{a}(u),
$$

and simple manipulation.

Using Lemma 20 in (5.28) we obtain

$$
\begin{aligned}
\left.f_{\text {scaled }}^{11}(X, Y)\right|_{a \rightarrow a+1}= & \frac{1}{2} K^{\text {hard }}(X, Y)-\frac{1}{4} Y^{-1 / 2} J_{a}\left(X^{1 / 2}\right) J_{a+1}\left(Y^{1 / 2}\right) \\
& +\frac{1}{2} \frac{\partial}{\partial Y} \int_{X}^{\infty} K^{\text {hard }}(t, Y) d t-\frac{1}{4} \frac{\partial}{\partial Y} J_{a}\left(Y^{1 / 2}\right) \int_{X}^{\infty} t^{-1 / 2} J_{a+1}\left(t^{1 / 2}\right) d t .
\end{aligned}
$$

In the special case $a=0$ the last integral can be evaluated, and this shows the final term cancels with the second term. We thus have agreement with (5.29) provided we can show

$$
\left.\frac{\partial}{\partial Y} \int_{0}^{\infty} K^{\text {hard }}(t, Y)\right|_{a=0} d t=0
$$


Now, using the integral representation from 4.10 ) shows

$$
\left.\int_{0}^{\infty} K^{\mathrm{hard}}(t, Y)\right|_{a=0} d t=\frac{1}{4} \int_{0}^{\infty} d t \int_{0}^{1} d s J_{0}(\sqrt{t s}) J_{0}(\sqrt{Y s})=\frac{1}{4} \int_{0}^{\infty} d t J_{0}(\sqrt{t}) \int_{0}^{1} d s \frac{1}{s} J_{0}(\sqrt{Y s})
$$

where the second equality follows by changing variables $t \mapsto t / s$. But

$$
\int_{0}^{\infty} J_{0}(\sqrt{t}) d t=2 \int_{0}^{\infty} s J_{0}(s) d s=2 \int_{0}^{\infty} \frac{d}{d s}\left(s J_{1}(s)\right) d s=0
$$

so indeed (5.31) holds true.

Let us now return to the consideration of $f_{\text {scaled }}^{11}$ in general. Previous studies have given formulas of a different form to (5.27) and (5.28). These read [17]

$$
\begin{aligned}
f_{\text {bulk }}^{11}(X, Y) & =K^{\text {bulk }}(X, Y) \\
f_{\text {soft }}^{11}(X, Y) & =K^{\text {soft }}(X, Y)+\frac{1}{2} \operatorname{Ai}(Y) \int_{-\infty}^{X} \operatorname{Ai}(t) d t \\
f_{\text {hard }}^{11}(X, Y) & =K^{\text {hard }}(X, Y)+\frac{J_{a+1}(\sqrt{Y})}{4 \sqrt{Y}} \int_{\sqrt{X}}^{\infty} J_{a-1}(u) d u
\end{aligned}
$$

where here $f_{\text {hard }}^{11}(X, Y)$ is for the scaled hard edge with singularity $x^{a / 2}$ as $x \rightarrow 0^{+}$. Agreement between (5.27) and (5.32), (5.33) is immediate upon substituting the integral formulas from (4.11), (4.12) in (5.27) and integrating by parts. It remains to show that the RHS of (5.34) agrees with the RHS of (5.28) with the replacement $a \mapsto a+1$. The verification is done by making use of Lemma 20 in (5.28) to obtain

$$
\begin{aligned}
\left.f_{\text {scaled }}^{11}(X, Y)\right|_{a \rightarrow a+1}= & \frac{1}{2} K^{\text {hard }}(X, Y)-\frac{1}{4 \sqrt{Y}} J_{a}(\sqrt{X}) J_{a+1}(\sqrt{Y})+\frac{1}{2} \frac{\partial}{\partial Y} \int_{X}^{\infty} \frac{Y}{t} K^{\text {hard }}(t, Y) d t \\
& -\frac{1}{4} \frac{\partial}{\partial Y} \sqrt{Y} J_{a+1}(\sqrt{Y}) \int_{X}^{\infty} t^{-1} J_{a}(\sqrt{t}) d t
\end{aligned}
$$

(c.f. $(5.30)$ ). Using the integral formula from (4.10) (without the restriction $a=0$ ), a straightforward calculation shows

$$
\frac{\partial}{\partial Y} \int_{X}^{\infty} \frac{Y}{t} K^{\mathrm{hard}}(t, Y) d t=K^{\mathrm{hard}}(X, Y)+\frac{J_{a}(\sqrt{Y})}{4} \int_{X}^{\infty} t^{-1} J_{a}(\sqrt{t}) d t .
$$

Substituting this in (5.35) gives agreement with (5.34) provided

$$
\begin{gathered}
-\frac{1}{4 \sqrt{Y}} J_{a}(\sqrt{X}) J_{a+1}(\sqrt{Y})+\frac{J_{a}(\sqrt{Y})}{8} \int_{X}^{\infty} t^{-1} J_{a}(\sqrt{t}) d t-\frac{1}{4} \frac{\partial}{\partial Y} \sqrt{Y} J_{a+1}(\sqrt{Y}) \int_{X}^{\infty} t^{-1} J_{a}(\sqrt{t}) d t \\
=\frac{J_{a+1}(\sqrt{Y})}{4 \sqrt{Y}} \int_{\sqrt{X}}^{\infty} J_{a-1}(u) d u .
\end{gathered}
$$

Since both sides vanish as $X \rightarrow \infty$, it suffices to check that the derivative with respect to $X$ of both sides agrees. This is easily verified using suitable Bessel function identities.

\subsection{The symplectic symmetry limit}

We know from (3.16) and (3.17) that in the limit $A \rightarrow-\infty$ the parameter dependent Laguerre and Jacobi ensembles tend to the LSE with weight $e^{-x}$ and the JSE with weight $(1-x)^{a+1}$ respectively. The results of the present study give that the corresponding $k$-point distribution is given by (1.31) with matrix elements specified by (3.99)-(3.101). As with the orthogonal symmetry limit, previous studies 
[40, 17, 1] have obtained the $k$-point distribution for the LSE with general $a>-1$, and the JSE with general $a, b>-1$. In particular, in terms of (5.1) and (5.2), the results of [1] give that the $k$-point distribution for the matrix ensembles $\mathrm{SE}_{n}\left(x^{a} e^{-x}\right)$ (LSE) and $\mathrm{SE}_{n}\left((1+x)^{b}(1-x)^{a+1}\right)$ (JSE), as specified by (1.15), are given by (1.31) with the $2 \times 2$ matrix $f$ in (1.31) having as its top left entry

$$
2 f^{11}(x, y)=\left(\frac{x}{y}\right)^{1 / 2} K_{2 n, a-1}^{L}(x, y)+\frac{2(2 n+a-1)}{h_{2 n, a-1}} y^{(a-2) / 2} e^{-y / 2} L_{2 n}^{a-1}(y) \int_{x}^{\infty} t^{(a-2) / 2} e^{-t / 2} L_{2 n-1}^{a-1}(t) d t
$$

in the Laguerre case, and

$$
\begin{aligned}
2 f^{11}(x, y)= & (1-x)\left(\frac{1+x}{1+y}\right)^{1 / 2} K_{2 n, a, b-1}^{J}(x, y)-\frac{2 n(2 n+b-2)}{h_{2 n-1, a, b-1}^{J}\left(2 n+\frac{1}{2}(a+b-2)\right)}(1-y)^{(a-1) / 2} \\
& \times(1+y)^{(b-2) / 2} P_{2 n}^{(a, b-1)}(y) \int_{x}^{1}(1-t)^{(a-1) / 2}(1+t)^{(b-2) / 2} P_{2 n-1}^{(a, b-1)}(t) d t
\end{aligned}
$$

in the Jacobi case (we have taken the transpose of the qdet formula in 迥, and thus have interchanged $x$ and $y$ in $f^{11}$ relative to the expression in [1]). The other entries in the matrix $f$ are related to $f^{11}$ by

$$
f^{22}(x, y)=f^{11}(y, x), \quad f^{12}(x, y)=-\int_{y}^{x} f^{11}(y, t) d t, \quad f^{21}(x, y)=\frac{\partial}{\partial y} f^{11}(y, x) .
$$

We see from the first equality in (3.65), (3.100) and (3.101) that the equations $(5.38)$ are identical to those obtained in Section 3.4 for the symplectic limit in the Laguerre case. Furthermore, in the Jacobi case, defining

$$
f^{22}(x, y)=\frac{1-y}{1-x} f^{22}(x, y)=\frac{1}{2}(1-y) K_{2 n}^{J}(x, y)+\frac{1}{2}\left((1-x) \frac{\partial}{\partial x}-1\right) \int_{y}^{1} K_{2 n}^{J}(t, x) d t
$$

the first equality in $(3.65)$ and $(3.99)-(3.101)$ together with simple scaling invariances of the quaternion determinant imply that the $k$-point correlation can be written as qdet $[\tilde{f}]$ with the elements of the $2 \times 2$ matrix $\tilde{f}$ given as in (5.38) but with each $f^{s s^{\prime}}$ replaced by $\tilde{f}^{s s^{\prime}}$. Thus it remains to show agreement between the first equality in (3.99), substituted in the first equation of (5.38), with (5.36) in the case $a=0$, and (5.39), substituted in the first equation of (5.38), with $(5.38)$ in the case $b=0$.

Consider first the Laguerre case. We know from [40, 17] that (5.36) can be rewritten to read

$$
2 f^{11}(x, y)=K_{2 n, a}^{L}(x, y)+G_{1}^{L}(y) G_{2}^{L}(x)
$$

where

$$
\begin{aligned}
G_{1}^{L}(y) & =\frac{1}{2 h_{2 n, a-1}^{L}} y^{a / 2-1} e^{-y / 2} L_{2 n}^{a-1}(y) \\
G_{1}^{L}(x) & =\left(2 x^{a / 2} e^{-x / 2} L_{2 n-1}^{a}(x)+(2 n+a-1) \int_{x}^{\infty} t^{a / 2-1} e^{-t / 2} L_{2 n-1}^{a-1}(t) d t\right) \\
& =\int_{0}^{x} t^{a / 2-1} e^{-t / 2}\left(2 n L_{2 n}^{a}(x)-(2 n+a) L_{2 n-1}^{a}(x)\right) .
\end{aligned}
$$

For the RHS of (3.99) to equal the RHS of (5.40) substituted in the first equality of (5.38) in the case $a=0$, by setting $y=0$ in both expressions we see that a necessary condition is that

$$
\frac{1}{2} K_{2 n}^{L}(x, 0)+\frac{1}{2} \frac{\partial}{\partial x} \int_{0}^{\infty} K_{2 n}^{L}(t, x) d x=K_{2 n, 0}^{L}(x, 0),
$$

which is obtained by setting $y=0$ in both expressions. This is just the previously established identity (3.88). Knowing that both sides agree at $y=0$, to show they are equal for all $y$ it suffices to show 
that their partial derivatives with respect to $y$ agree. In fact equating the partial derivatives gives the previously established identity (5.13).

To show agreement in the Jacobi case, we require the analogue of (5.40).

Lemma 21. The formula (5.37) has the alternative form

$$
2 f^{11}(x, y)=(1-x) K_{2 n, a, b}^{J}(x, y)+G_{1}^{J}(y) G_{2}^{J}(x)
$$

where

$$
\begin{aligned}
G_{1}(y)= & \frac{4 n}{4 n+a+b-1} \frac{1}{h_{2 n-1, a, b}^{J}}(1-y)^{(a-1) / 2}(1+y)^{b / 2-1} P_{2 n}^{(a, b-1)}(y) \\
G_{2}(y)= & -\left\{P_{2 n-1}^{(a, b)}(x)(1-x)^{(a+1) / 2}(1+x)^{b / 2}\right. \\
& \left.+(b+2 n-1) \int_{x}^{1} d t(1-t)^{(a-1) / 2}(1+t)^{(b-2) / 2} P_{2 n-1}^{(a, b-1)}(t)\right\} \\
= & \frac{a+b+2 n}{4} \int_{-1}^{x}(1-t)^{(a-1) / 2}(1+t)^{b / 2} P_{2 n-1}^{(a, b+1)}(t) d t .
\end{aligned}
$$

Proof. This is derived in a similar fashion to the result of Lemma 18 .

With (5.41) substituted in the first equality of (5.38), agreement of the resulting expression with (5.39) in the case $b=0$ can be established by first checking that both expressions coincide at $y=-1$ (this follows from (3.69) $)$, and then showing both expressions have the same partial derivative with respect to $y$ (this follows from (5.15)).

Next we consider the form of the scaled matrix elements of Propositions 16 and 17 as $\alpha \rightarrow-\infty$, which corresponds to the symplectic limit. Integration by parts shows

$$
\begin{aligned}
\frac{Y}{X} f_{\text {scaled }}^{22}(X, Y) & \sim \tilde{f}_{\text {scaled }}^{22}(X, Y)=\frac{1}{2} \sqrt{\frac{Y}{X}} K^{\text {scaled }}(X, Y)+\frac{1}{2} \int_{0}^{Y} \frac{1}{\sqrt{u}} \frac{\partial}{\partial X} K^{\text {scaled }}(X, t) \\
\frac{8}{\alpha^{2}} f_{\text {scaled }}^{12}(X, Y) & \sim \int_{X}^{\infty} \tilde{f}_{\text {scaled }}^{22}(t, Y) d t \\
\frac{\alpha^{2}}{8} f_{\text {scaled }}^{21}(X, Y) & \sim \frac{\partial}{\partial Y} \tilde{f}_{\text {scaled }}^{22}(X, Y)
\end{aligned}
$$

in the case of the soft edge and the bulk, and

$$
\begin{aligned}
& \frac{Y}{X} f_{\text {hard }}^{22}(X, Y) \sim \tilde{f}_{\text {hard }}^{22}(X, Y)=\frac{1}{2} \sqrt{\frac{Y}{X}} K^{\text {hard }}(X, Y)+\frac{1}{2} \int_{0}^{Y} \frac{1}{\sqrt{u}} \frac{\partial}{\partial X} \sqrt{X} K^{\text {hard }}(X, t) d t \\
& \frac{8}{\alpha^{2}} X Y f_{\text {scaled }}^{12}(X, Y) \sim-\int_{0}^{X} f_{\text {hard }}^{22}(t, Y) d t \\
& \frac{\alpha^{2}}{8} \frac{1}{X Y} f_{\text {hard }}^{21}(X, Y) \sim \frac{\partial}{\partial Y} \tilde{f}_{\substack{\text { hard } \\
\alpha=-\infty}}^{22}(X, Y)
\end{aligned}
$$

in the case of the hard edge with singularity $x^{a+1}$ as $x \rightarrow 0$.

Previous studies have given formulas of a different form to (5.42) and (5.43), these being 17

$$
\begin{aligned}
f_{\text {bulk }}^{22}(X, Y) & =K^{\text {bulk }}(X, Y) \\
f_{\text {soft }}^{22}(X, Y) & =K^{\text {soft }}(X, Y)-\frac{1}{2} \operatorname{Ai}(X) \int_{Y}^{\infty} \operatorname{Ai}(s) d s \\
f_{\text {hard }}^{22}(X, Y) & =K^{\text {hard }}(X, Y)-\frac{1}{4 \sqrt{X}} J_{a-1}(\sqrt{X}) \int_{0}^{\sqrt{Y}} J_{a+1}(s) d s
\end{aligned}
$$


where here $f_{\text {hard }}^{22}$ relates to the scaled hard edge with singularity $x^{a}$ as $x \rightarrow 0^{+}$. The verification that these formulas are identical to $(5.42)$ or $(5.43)$ is done in the same way as reconciling (5.27)-(5.28) with (5.32) and (5.34).

\subsection{Bulk structure function for the decimated orthogonal ensemble with a parameter}

In general the 2-point correlation implied by (1.31) is given in terms of the corresponding 1-point correlation (the density) and the matrix elements $f^{s s^{\prime}}\left(s, s^{\prime}=1,2\right)$ of $f$ according to

$$
\rho_{2}\left(x_{1}, x_{2}\right)=\rho_{1}\left(x_{1}\right) \rho_{1}\left(x_{2}\right)-\left(f^{11}\left(x_{1}, x_{2}\right) f^{22}\left(x_{1}, x_{2}\right)-f^{12}\left(x_{1}, x_{2}\right) f^{21}\left(x_{1}, x_{2}\right)\right) .
$$

Now the truncated quantity

$$
\rho_{2}^{T}\left(x_{1}, x_{2}\right):=\rho_{2}\left(x_{1}, x_{2}\right)-\rho_{1}\left(x_{1}\right) \rho_{1}\left(x_{2}\right)
$$

decays for large $\left|x_{1}-x_{2}\right|$. Furthermore, in the bulk it is a function only of the difference $\left|x_{1}-x_{2}\right|$. Thus in the bulk the Fourier transform of $\rho_{2}^{T}\left(x_{1}-x_{2}, 0\right)$ is a well defined quantity. We seek its evaluation in the case of the parameter dependent orthogonal ensemble with a parameter, when the matrix elements are specified by (4.27)-(4.29). Explicitly, we will compute the quantity

$$
\begin{aligned}
S(k) & =1+\int_{-\infty}^{\infty} \rho_{2}^{T}(x) e^{i k x} d x \\
& =1-\int_{-\infty}^{\infty}\left(f^{11}(x) f^{22}(-x)+f^{12}(x) f^{21}(-x)\right) e^{i k x} d x
\end{aligned}
$$

where to obtain the second equality we have substituted (5.44) and made use of the facts, apparant from (4.27)-(4.29), that $f^{11}(x), f^{22}(x)$ are even functions of $x$, while $f^{12}, f^{21}$ are odd functions of $x$. Following [16] we know that the most efficient way to compute such a Fourier transform is to make use of the general formula

$$
\int_{-\infty}^{\infty} f(x) f(-x) e^{i k x} d x=\frac{1}{2 \pi} \int_{-\infty}^{\infty} \hat{f}(l) \hat{f}(l-k) d l, \quad \hat{f}(l):=\int_{-\infty}^{\infty} f(x) e^{i l x} d x .
$$

Thus we must first compute the Fourier transform of the individual matrix elements.

The calculation of the Fourier transform of the individual matrix elements is simplified by first noting, making use of the fact that $K^{\text {bulk }}(X, Y)$ is a function of $X-Y$, that the expression (4.27) for $f_{\text {bulk }}^{22}(X, Y)$ can be simplified to read

$$
f_{\text {bulk }}^{22}(X, Y)=K^{\text {bulk }}(X, Y),
$$

independent of the parameter $\alpha$. Recalling the first equality in (3.65), and the integral representation of (4.11), we thus have

$$
\hat{f}_{\text {bulk }}^{22}(l)=\hat{f}_{\text {bulk }}^{11}(l)=\chi_{|l|<\pi} .
$$

Furthermore, substituting the integral representation of (4.11) in 4.28)-(4.29) allows us to compute

$$
\hat{f}_{\text {bulk }}^{12}(l)=\frac{(\alpha / 2)^{2}+l^{2}}{2 i l} \chi_{|l|<\pi}, \quad \hat{f}_{\text {bulk }}^{21}(l)=-\frac{2 i l}{(\alpha / 2)^{2}+l^{2}} \chi_{|l|>\pi} .
$$

Thus

$$
\begin{aligned}
\int_{-\infty}^{\infty} \hat{f}_{\mathrm{bulk}}^{11}(l) \hat{f}_{\mathrm{bulk}}^{22}(l-k) d l & =\int_{-\pi}^{\pi} \chi_{|l-k|<\pi} d l= \begin{cases}2 \pi-|k|, & |k|<2 \pi \\
0, & |k| \geq 2 \pi\end{cases} \\
\int_{-\infty}^{\infty} \hat{f}^{12}(l) \hat{f}^{21}(l-k) d l & =-\int_{-\pi-|k|}^{\min (\pi-|k|,-\pi)} \frac{(\alpha / 2)^{2}+(l+|k|)^{2}}{l+|k|} \frac{l}{(\alpha / 2)^{2}+l^{2}} d l .
\end{aligned}
$$


The integral (5.49) can be evaluated in terms of elementary functions, with there being a different functional form for $|k|<2 \pi,|k| \geq 2 \pi$. Substituting this and (5.48) in (5.46) (appropriately rewritten using (5.47), we find that for $|k|<2 \pi$

$$
\begin{aligned}
S(k)= & \frac{|k|}{\pi}+\frac{|k|}{4 \pi\left((\alpha / 2)^{2}+k^{2}\right)}\left[\alpha|k|\left(\arctan \frac{2 \pi}{\alpha}-\arctan \frac{2|k|+2 \pi}{\alpha}\right)\right. \\
& \left.-\left(\frac{\alpha^{2}}{2}+k^{2}\right) \log \left(\frac{(\alpha / 2)^{2}+(|k|+\pi)^{2}}{(\alpha / 2)^{2}+\pi^{2}}\right)-\frac{\alpha^{2}}{2} \log |1-| k|/ \pi|\right],
\end{aligned}
$$

while for $|k| \geq 2 \pi$

$$
\begin{aligned}
S(k)= & 2+\frac{\alpha k^{2} \arctan (2(|k|-\pi) / \alpha)}{4 \pi\left((\alpha / 2)^{2}+k^{2}\right)}-\frac{\alpha k^{2} \arctan (2(|k|+\pi) / \alpha)}{4 \pi\left((\alpha / 2)^{2}+k^{2}\right)} \\
& -\frac{\left(2(\alpha / 2)^{2}|k|+|k|^{3}\right)}{4 \pi\left((\alpha / 2)^{2}+k^{2}\right)} \log \frac{(\alpha / 2)^{2}+(|k|+\pi)^{2}}{(\alpha / 2)^{2}+(|k|-\pi)^{2}} .
\end{aligned}
$$

Of particular interest is the small $|k|$ expansion of (5.50). We find

$$
S(k)=\frac{|k|}{\pi}+\frac{1}{2 \pi^{2}} \frac{(\alpha / 2)^{2}-\pi^{2}}{(\alpha / 2)^{2}+\pi^{2}} k^{2}+\frac{\left((\alpha / 2)^{2}-\pi^{2}\right)^{2}}{4 \pi^{3}\left((\alpha / 2)^{2}+\pi^{2}\right)^{2}}|k|^{3}+\mathrm{O}\left(k^{4}\right) .
$$

We see from (5.52) that the coefficient of the leading order term, proportional to $|k|$, in the small $|k|$ expansion of $S(k)$ is independent of the parameter $\alpha$, and has the value $1 / \pi$. This is to anticipated from the interpretation of (1.4) as a one-component log-potential Coulomb system with coupling $\beta=1$. The coupling within pairs can be regarded as a short range potential which should not affect properties determined by the long-ranged logarithmic potential. One such property is the behaviour

$$
S(k) \underset{|k| \rightarrow 0}{\sim} \frac{|k|}{\pi \beta}
$$

for a one-component log-potential system with coupling $\beta$ (see e.g. [16]), thus implying the leading behaviour seen in (5.52).

\subsection{Distribution of odd labelled coordinates for a special parameter}

In the Introduction, attention was drawn to the properties (1.6), (1.20) of the parameter dependent ensembles (1.4), (1.16) relating to the distribution of the even labelled coordinates. Similarly, we noted the properties (1.10), (1.19) of the even labelled coordinates in the superimposed parameter dependent ensembles (1.8), (1.17). For the special value of the parameter for which the one body factor for the even labelled coordinates reduces to a constant, it turns out that the odd labelled coordinates also have a distribution which coincides with that of other matrix ensembles. This follows from the following integration formulas.

Lemma 22. Let $x_{1}, x_{2}, \ldots, x_{2 n}$ be ordered as in (1.5), and label this ordering $X$. We have

$$
\int_{X} d x_{2} d x_{4} \cdots d x_{2 n} \prod_{1 \leq j<k \leq 2 n}\left(x_{j}-x_{k}\right)=\frac{1}{(2 n) !} \prod_{j=1}^{n} x_{2 j-1}^{2} \prod_{1 \leq j<k \leq n}\left(x_{2 j-1}-x_{2 k-1}\right)^{4}
$$

and

$$
\int_{X} d x_{2} d x_{4} \cdots d x_{2 n} \prod_{1 \leq j<k \leq n}\left(x_{2 j}-x_{2 k}\right)=\frac{1}{n !} \prod_{j=1}^{n} x_{2 j-1} \prod_{1 \leq j<k \leq n}\left(x_{2 j-1}-x_{2 k-1}\right) .
$$


Proof. To derive (5.53), we use the Vandermonde determinant formula to write

$$
\prod_{1 \leq j<k \leq 2 n}\left(x_{j}-x_{k}\right)=\operatorname{det}\left[x_{2 n+1-j}^{k-1}\right]_{j, k=1, \ldots, 2 n} .
$$

The method of integration over alternative variables gives

$$
\begin{aligned}
& \int_{X} d x_{2} d x_{4} \cdots d x_{2 n} \prod_{1 \leq j<k \leq 2 n}\left(x_{j}-x_{k}\right)=\operatorname{det}\left[\begin{array}{c}
\frac{1}{k} x_{2 n+1-2 j}^{k} \\
x_{2 n+1-2 j}^{k-1}
\end{array}\right]_{\substack{j=1, \ldots, n \\
k=1, \ldots, 2 n}}=\frac{1}{(2 n) !} \prod_{j=1}^{n} x_{2 j-1}^{2} \operatorname{det}\left[\begin{array}{c}
x_{2 n+1}^{k-1} \\
(k-1) x_{2 n+1-2 j}^{k-2 j}
\end{array}\right]_{\substack{j=1, \ldots, n \\
k=1, \ldots, 2 n}} .
\end{aligned}
$$

This final determinant is well known to be equal to the product of differences to the fourth power, and thus (5.53) follows.

A similar, even simpler, computation gives (5.54).

We remark that (5.53) and (5.54) are equivalent to the special case of (1.12) and (1.14) in which $(f, g)$ is given by the Jacobi weight in (1.13) with $a=1$.

For the ensembles (1.4), (1.16), it follows immediately from (5.53) that

$$
\operatorname{odd}\left(\mathrm{OE}_{2 n}\left(f_{\mathrm{o}}, f_{\mathrm{e}}\right)\right)=\mathrm{SE}_{n}(h)
$$

with

$$
\left(f_{\mathrm{o}}, f_{\mathrm{e}}, h\right)=\left\{\begin{array}{l}
\left.\left(e^{-x / 2} e^{A x / 2}, e^{-x / 2} e^{-A x / 2}, x^{2} e^{-x}\right)\right|_{A=-1}(x>0) \\
\left.\left((1-x)^{(a-A-1) / 2},(1-x)^{(a+A-1) / 2},(1+x)^{2}(1-x)^{a-1}\right)\right|_{A=1-a}(-1<x<1)
\end{array}\right.
$$

in the Laguerre and Jacobi cases respectively. Similarly, for the ensembles (1.8), (1.17), it follows immediately from (5.54) that

$$
\operatorname{odd}\left(\mathrm{OE}_{n}\left(f_{\mathrm{o}}, f_{\mathrm{e}}\right) \cup \mathrm{OE}_{n}\left(f_{\mathrm{o}}, f_{\mathrm{e}}\right)\right)=\mathrm{UE}_{n}(\tilde{h})
$$

where $f_{\mathrm{o}}, f_{\mathrm{e}}$ are as in (5.56), while

$$
\tilde{h}= \begin{cases}x e^{-x}, & \text { Laguerre case } \\ (1+x)(1-x)^{a-1}, & \text { Jacobi case }\end{cases}
$$

A consequence of (5.55) and (5.57) is that the $k$-point odd-odd correlation for the ensemble on the LHS must coincide with the $k$-point correlation for the ensemble on the RHS. This is simple to explicitly verify for the relation (5.57). Then the $k$-point distribution on the RHS is given by

$$
\operatorname{det}\left[K_{n, 1}^{L}\left(x_{j}, x_{l}\right)\right]_{j, l=1, \ldots, k}, \quad \operatorname{det}\left[\left(1-x_{j}\right) K_{n, a-1,1}^{J}\left(x_{j}, x_{l}\right)\right]_{j, l=1, \ldots, k}
$$

in the Laguerre and Jacobi cases respectively. On the LHS the odd-odd $k$-point correlation is given by

$$
\operatorname{det}\left[\left.K_{\mathrm{oo}}^{L}\left(x_{j}, x_{l}\right)\right|_{A=-1}\right]_{j, l=1, \ldots, k}, \quad \operatorname{det}\left[\left(1-x_{j}\right) K_{\mathrm{oo}}^{J}\left(x_{j}, x_{l}\right)\right]_{j, l=1, \ldots, k},
$$

where $K_{\mathrm{oo}}^{L}, K_{\mathrm{oo}}^{J}$ are given by (2.15) and (2.16) respectively. Using appropriate Laguerre and Jacobi polynomial formulas to evaluate

$$
\int_{0}^{x^{\prime}} L_{l}(x) d x, \quad \frac{d}{d x} e^{-x} L_{l}(x), \quad \int_{0}^{x^{\prime}} P_{l}^{(a, 0)}(x) d x, \quad \frac{d}{d x}(1-x)^{a} P_{l}^{(a, 0)}(x)
$$

it is readily seen that (5.60) can be reduced to (5.59). 


\subsection{Gap probabilities and eigenvalue distributions}

Observable quantities for eigenvalue sequences closely related to correlation functions are gap probabilities and distribution functions of individual eigenvalues. The gap probability specifies the probability - to be denoted $E(p ; I ; \mathrm{ME})$ - that an interval $I$ (the gap) in a given matrix ensemble ME contains precisely $p$ eigenvalues. With the eigenvalues ordered as in (1.5), we may choose to observe eigenvalues of a definite parity, even or odd labelled. In this case we denote the gap probability as $E^{(\cdot)}(p ; I ; \mathrm{ME}),(\cdot)=(\mathrm{e})$ ven, (o)dd, where $p$ now refers to eigenvalues of parity $(\cdot)$ only. For $I=(s, \infty)$ with $s$ inside the support of $\mathrm{ME}$, it follows from the ordering (1.5) that

$$
\begin{aligned}
& E^{(\mathrm{o})}(p ;(s, \infty) ; \mathrm{ME})=(E(2 p-1 ;(s, \infty) ; \mathrm{ME})+E(2 p ;(s, \infty) ; \mathrm{ME})) \\
& E^{(\mathrm{e})}(p ;(s, \infty) ; \mathrm{ME})=(E(2 p ;(s, \infty) ; \mathrm{ME})+E(2 p+1 ;(s, \infty) ; \mathrm{ME}))
\end{aligned}
$$

where $E(-1 ; I ; \mathrm{ME}):=0$. Consequently we can express $E(p ;(s, \infty) ; \mathrm{ME})$ in terms of $\left\{E^{(\cdot)}(p ;(s, \infty) ; \mathrm{ME})\right\}$,

$$
\begin{aligned}
E(2 p ;(s, \infty) ; \mathrm{ME}) & =\sum_{j=0}^{p} E^{(\mathrm{o})}(j ;(s, \infty) ; \mathrm{ME})-\sum_{j=0}^{p-1} E^{(\mathrm{e})}(j ;(s, \infty) ; \mathrm{ME}) \\
E(2 p+1 ;(s, \infty) ; \mathrm{ME}) & =\sum_{j=0}^{p} E^{(\mathrm{e})}(j ;(s, \infty) ; \mathrm{ME})-\sum_{j=0}^{p} E^{(\mathrm{o})}(j ;(s, \infty) ; \mathrm{ME}) .
\end{aligned}
$$

We will denote the PDF for the distribution function of the $k$ th eigenvalue $x_{k}$ (with the ordering (1.5)) by $p\left(k-1 ; s ; \mathrm{ME}\right.$ ) (here $k-1$ is the number of eigenvalues greater than $x_{k}$ ). A standard formula (see e.g. 113]) gives that

$$
p(k-1 ; s ; \mathrm{ME})=\frac{d}{d s} E(k-1 ;(s, \infty) ; \mathrm{ME})+p(k-2 ; s ; \mathrm{ME}), \quad k \geq 1
$$

where $p(-1 ; s ; \mathrm{ME}):=0$, so knowledge of $\{E(p ;(s, \infty) ; \mathrm{ME})\}_{p=0, \ldots, k-1}$ suffices to compute $p(k-1 ; s ; \mathrm{ME})$.

Let us consider first the gap probabilities and eigenvalue distributions for $\mathrm{ME}=\left(\mathrm{LOE}_{n} \cup \mathrm{LOE}_{n}\right)^{A}$ and $\mathrm{ME}=\left(\mathrm{JOE}_{n} \cup \mathrm{JOE}_{n}\right)^{A}$ (i.e. the PDFs (1.8) and (1.16)). The identities (1.12) and (1.18) tell us that

$$
\begin{aligned}
E^{(\mathrm{e})}\left(p ;(s, \infty) ;\left(\mathrm{LOE}_{n} \cup \mathrm{LOE}_{n}\right)^{A}\right) & =E\left(p ;(s, \infty) ;\left.\mathrm{LUE}_{n}\right|_{a=0}\right), \\
E^{(\mathrm{e})}\left(p ;(s, 1) ;\left(\mathrm{JOE}_{n} \cup \mathrm{JOE}_{n}\right)^{A}\right) & =E\left(p ;(s, 1) ;\left.\mathrm{JUE}_{n}\right|_{b=0}\right) \\
p\left(2 k-1 ; s ;\left(\mathrm{LOE}_{n} \cup \mathrm{LOE}_{n}\right)^{A}\right) & =p\left(k-1 ; s ;\left.\mathrm{LUE}_{n}\right|_{a=0}\right) \\
p\left(2 k-1 ; s ;\left(\mathrm{JOE}_{n} \cup \mathrm{JOE}_{n}\right)^{A}\right) & =p\left(k-1 ; s ;\left.\mathrm{JUE}_{n}\right|_{b=0}\right)
\end{aligned}
$$

which we can check are consistent with (5.3). Note in particular that each quantity in (5.4) is independent of the parameter $A$, and we remark too that each has a known Painlevé transcendent evaluation [38, 20, 21]. To specify $E^{(o)}$ and thus $p(2 k ; s ; \mathrm{ME}), E$ for these matrix ensembles we make use of the standard formula relating the gap probability to the correlation functions,

$$
E^{(\mathrm{o})}(p ; I ; \mathrm{ME})=\left.\frac{(-1)^{p}}{p !} \frac{\partial^{p}}{\partial \xi^{p}}\left(1+\sum_{k=1}^{n} \frac{(-\xi)^{k}}{k !} \int_{I} d x_{1} \cdots \int_{I} d x_{k} \rho_{(k)}^{(\mathrm{o})}\left(x_{1}, \ldots, x_{k}\right)\right)\right|_{\xi=1} .
$$

We know from (1.24) that $\rho_{(k)}^{(\mathrm{o})}$ is the $k \times k$ determinant $\operatorname{det}\left[K_{\mathrm{oo}}\left(x_{i}, x_{j}\right)\right]_{i, j=1, \ldots, k}$. In this circumstance the expression in brackets is just the expansion [39] of the Fredholm determinant of the integral operator $K_{\text {oo }}$ with kernel $K_{\text {oo }}(x, y)$ supported on $I$,

$$
E^{(\mathrm{o})}(p ; I ; \mathrm{ME})=\left.\frac{(-1)^{p}}{p !} \frac{\partial^{p}}{\partial \xi^{p}} \operatorname{det}\left(\mathbf{1}-\xi K_{\mathrm{oo}}\right)\right|_{\xi=1}
$$


(here 1 denotes the identity operator). When $p=0$ this reads

$$
E^{(\mathrm{o})}(0 ; I ; \mathrm{ME})=E(0 ; I ; \mathrm{ME})=\operatorname{det}\left(\mathbf{1}-K_{\mathrm{oo}}\right)
$$

where the first equality follows from the first equation of (5.1). Using the fact that with $A=0,\left(\mathrm{LOE}_{n} \cup\right.$ $\left.\mathrm{LOE}_{n}\right)^{A}$ and $\left(\mathrm{JOE}_{n} \cup \mathrm{JOE}_{n}\right)^{A}$ reduce to $\mathrm{LOE}_{n} \cup \mathrm{LOE}_{n}$ and $\mathrm{JOE}_{n} \cup \mathrm{JOE}_{n}$ respectively, it follows that $E\left(0 ; I ;\left.\mathrm{ME}\right|_{A=0}\right)=(E(0 ; I ; \mathrm{OE}))^{2}$ and thus we deduce from 5.7$)$ that

$$
\left(E\left(0 ;(s, \infty) ;\left.\mathrm{LOE}\right|_{a=0}\right)\right)^{2}=\operatorname{det}\left(\mathbf{1}-\left.K_{\mathrm{oO}}^{L}\right|_{A=0}\right), \quad\left(E\left(0 ;(s, \infty) ;\left.\mathrm{JOE}\right|_{\substack{a \mapsto(a-1) / 2 \\ b=0}}\right)\right)^{2}=\operatorname{det}\left(\mathbf{1}-\left.K_{\mathrm{oO}}^{J}\right|_{A=0}\right) .
$$

We remark that $E\left(0 ;(s, \infty) ;\left.\mathrm{LOE}\right|_{a=0}\right)$ has recently been evaluated in terms of Painlevé transcendents 2. 21.

Let us now consider the gap probabilities and eigenvalue distributions for $\mathrm{ME}=\left(\mathrm{LOE}_{2 n}\right)^{A}$ and $\mathrm{ME}=\left(\mathrm{JOE}_{2 n}\right)^{A}$ (i.e. the PDFs (1.4) and (1.16)). The identities (1.14) and (1.19) tell us that

$$
\begin{aligned}
E^{(\mathrm{e})}\left(p ;(s, \infty) ;\left(\mathrm{LOE}_{2 n}\right)^{A}\right) & =E\left(p ;(s, \infty) ;\left.\mathrm{LSE}_{n}\right|_{a=0}\right) \\
E^{(\mathrm{e})}\left(p ;(s, 1) ;\left(\mathrm{JOE}_{2 n}\right)^{A}\right) & =E\left(p ;(s, 1) ;\left.\mathrm{JSE}_{n}\right|_{\substack{a \rightarrow a+1 \\
b=0}}\right) \\
p\left(2 k-1 ; s ;\left(\mathrm{LOE}_{2 n}\right)^{A}\right) & =p\left(k-1 ; s ;\left.\mathrm{LSE}_{n}\right|_{a=0}\right), \\
p\left(2 k-1 ; s ;\left(\mathrm{JOE}_{n} \cup \mathrm{JOE}_{n}\right)^{A}\right) & =p\left(k-1 ; s ;\left.\mathrm{JSE}_{n}\right|_{\substack{a \mapsto a+1 \\
b=0}}\right),
\end{aligned}
$$

(c.f. (5.4)). Of these quantities $E\left(0 ;(s, \infty) ;\left.\operatorname{LSE}_{n}\right|_{a=0}\right)$ and $p\left(0 ; s ;\left.\operatorname{LSE}_{n}\right|_{a=0}\right)$ are known in terms of Painlevé transcendents [2, 21]. To specify $E^{(\mathrm{o})}$ we again make use of (5.5), this time noting from (1.27) that $\rho_{(k)}^{(\mathrm{o})}$ is the $k \times k$ quaternion determinant $q \operatorname{det}\left[f_{\mathrm{oo}}\left(x_{i}, x_{j}\right)\right]_{i, j=1, \ldots, k}$ where $f_{\mathrm{oo}}$ is the $2 \times 2$ matrix representation of a particular real quanternion, which in turn implies

$$
E^{(\mathrm{o})}(p: I ; \mathrm{ME})=\left.\frac{(-1)^{p}}{p !} \frac{\partial^{p}}{\partial \xi^{p}} \operatorname{qdet}\left(\mathbf{1}-\xi \mathrm{f}_{\mathrm{oo}}\right)\right|_{\xi=1}
$$

(with $\left\{\lambda_{j}\right\}$ denoting the distinct eigenvalues of $f_{\mathrm{oo}}, \operatorname{qdet}\left(\mathbf{1}-\xi f_{\mathrm{oo}}\right)=\prod_{j}\left(1-\xi \lambda_{j}\right)$ ). We remark that for general $A, E^{(\mathrm{o})}\left(0 ;(s, \infty),(\mathrm{LOE})_{2 n}^{A}\right)$ has recently been evaluated in terms of Painlevé transcendents [2].

All the above formulas have well defined scaled limits. In particular

$$
\begin{aligned}
& \lim _{n \rightarrow \infty} E^{(\mathrm{o})}\left(p ;\left(4 n+2(2 n)^{1 / 3} s, \infty\right) ;\left(\mathrm{LOE}_{n} \cup \mathrm{LOE}_{n}\right)^{A=\alpha / 2(2 n)^{1 / 3}}\right) \\
& :=E^{(\mathrm{o}) \operatorname{soft}}\left(p ;(s, \infty) ;(\mathrm{OE} \cup \mathrm{OE})^{\alpha}\right)=\left.\frac{(-1)^{p}}{p !} \frac{\partial^{p}}{\partial \xi^{p}} \operatorname{det}\left(\mathbf{1}-\xi K_{\mathrm{oo}}^{\mathrm{soft}}\right)\right|_{\xi=1} \\
& \lim _{n \rightarrow \infty} E^{(\mathrm{o})}\left(p ;\left(1-\frac{s^{2}}{2 n^{2}}, 1\right) ;\left(\mathrm{JOE}_{n} \cup \mathrm{JOE}_{n}\right)^{A=4 n^{2} \alpha}\right) \\
& :=E^{(\mathrm{o}) \operatorname{hard}}\left(p ;(0, s) ;(\mathrm{OE} \cup \mathrm{OE})^{\alpha, a}\right)=\left.\frac{(-1)^{p}}{p !} \frac{\partial^{p}}{\partial \xi^{p}} \operatorname{det}\left(\mathbf{1}-\xi K_{\mathrm{oo}}^{\mathrm{hard}}\right)\right|_{\xi=1}
\end{aligned}
$$

(for the justification of the limiting processes see [7]). An evaluation of $E^{(\mathrm{o}) \operatorname{soft}}\left(p ;(s, \infty) ;(\mathrm{OE} \cup \mathrm{OE})^{\alpha}\right)$ in terms of a Riemann-Hilbert problem and Painlevé II transcendents has been given in [5].

\section{Acknowledgements}

We thank J. Baik for encouraging us to take up the problem of calculating the correlation functions for (1.4). The work of PJF was supported by the Australian Research Council. 


\section{References}

[1] M. Adler, P.J. Forrester, T. Nagao, and P. van Moerbeke. Classical skew orthogonal polynomials and random matrices. J. Stat. Phys., 99:141-170, 2000.

[2] J. Baik. Painlevé expressions for LOE, LSE and interpolating ensembles. Int. Math. Res. Notices, 33:1739-1789, 2002.

[3] J. Baik, P. Dieft, and K. Johansson. On the distribution of the length of the longest increasing subsequence of random permutations. J. Amer. Math. Soc., 12:1119-1178, 1999.

[4] J. Baik and E.M. Rains. Algebraic aspects of increasing subsequences. Duke Math. J., 109:1-65, 2001.

[5] J. Baik and E.M. Rains. The asymptotics of monotone subsequences of involutions. Duke Math. J., 109:205-281, 2001.

[6] J. Baik and E.M. Rains. Symmetrized random permutations. In P.M. Bleher and A.R. Its, editors, Random matrix models and their applications, volume 40 of Mathematical Sciences Research Institute Publications, pages 171-208. Cambridge University Press, United Kingdom, 2001.

[7] A. Borodin and P.J. Forrester. Increasing subsequences and the hard-to-soft edge transition in matrix ensembles. arXive:math-ph/0205007, 2002.

[8] A. Borodin and G. Olshanski. $z$-measures on partitions, Robinson-Schensted-Knuth correspondence, and $\beta=2$ random matrix ensembles. In P.M. Bleher and A.R. Its, editors, Random matrix models and their applications, volume 40 of Mathematical Sciences Research Institute Publications, pages 171-208. Cambridge University Press, United Kingdom, 2001.

[9] F.J. Dyson. Statistical theory of energy levels of complex systems I. J. Math. Phys., 3:140-156, 1962.

[10] F.J. Dyson. Statistical theory of energy levels of complex systems III. J. Math. Phys., 3:166-175, 1962.

[11] F.J. Dyson. Correlations between the eigenvalues of a random matrix. Commun. Math. Phys., 19:235-250, 1970.

[12] E. Dueñez. Random matrix ensembles associated to compact symmetric spaces. arXive math$\mathrm{ph} / 0111005$.

[13] P.J. Forrester. Log-gases and Random Matrices. www.ms.unimelb.edu.au/ ${ }^{\sim}$ matpjf/matpjf.html.

[14] P.J. Forrester. The spectrum edge of random matrix ensembles. Nucl. Phys. B, 402:709-728, 1993.

[15] P.J. Forrester. Random walks and random permutations. J. Phys. A, 34:L417-L423, 2001.

[16] P.J. Forrester, B. Jancovici, and D.S. McAnally. Analytic properties of the structure function for the one-dimensional one-component log-gas. J. Stat. Phys., 102:737-780, 2001.

[17] P.J. Forrester, T. Nagao, and G. Honner. Correlations for the orthogonal-unitary and symplecticunitary transitions at the hard and soft edges. Nucl. Phys. B, 553:601-643, 1999.

[18] P.J. Forrester and E.M. Rains. Inter-relationships between orthogonal, unitary and symplectic matrix ensembles. In P.M. Bleher and A.R. Its, editors, Random matrix models and their applications, volume 40 of Mathematical Sciences Research Institute Publications, pages 171-208. Cambridge University Press, United Kingdom, 2001. 
[19] P.J. Forrester and E.M. Rains. Interpretations of some parameter dependent generalizations of classical matrix ensembles. preprint, 2002.

[20] P.J. Forrester and N.S. Witte. Application of the $\tau$-function theory of Painlevé equations to random matrices: PV, PIII, the LUE, JUE and CUE. Commun. Pure Appl. Math., 55:679-727, 2002.

[21] P.J. Forrester and N.S. Witte. Application of the $\tau$-function theory of Painlevé equations to random matrices: PVI, the JUE,CyUE, cJUE and scaled limits. math-phy/0204008, 2002.

[22] K. Frahm and J.L. Pichard. Brownian motion ensembles and parametric correlations of the transmission eigenvalues: applications to coupled quantum billiards and to disordered systems. J. Phys. I (France), 5:877-906, 1995.

[23] I.S. Gradshteyn and I.M. Ryzhik. Tables of Integrals, Series and Products. Academic Press, New York, 4th edition, 1980.

[24] J. Gunson. Proof of a conjecture of Dyson in the statistical theory of energy levels. J. Math. Phys., $4: 752-753,1962$.

[25] A.J. Guttmann, A.L. Owczarek, and X.G. Viennot. Vicious walkers and Young tableaux i: without walls. J. Phys. A, 31:8123-8135, 1998.

[26] K. Hikami and T. Imamura. Vicious walkers and hook Young tableaux. arXive:cond-mat/0209512, 2002.

[27] K. Johansson. Shape fluctuations and random matrices. Commun. Math. Phys., 209:437-476, 2000.

[28] K. Johansson. Non-intersecting paths, random tilings and random matrices. Prob. Theory Rel., 123:225-280, 2002.

[29] I.M. Johnstone. On the distribution of the largest principal component. Ann. Stat., 29:295-327, 2001.

[30] C. Krattenthaler. The major counting of nonintersecting lattice paths and generating functions for tableaux. number 552 of Memoirs of the American Mathematical Society. American Mathematical Society, Providence, Rhode Island, 1995.

[31] G. Mahoux and M.L. Mehta. A method of integration over matrix variables IV. J. Physique I (France), 1:1093-1108, 1991.

[32] M.L. Mehta and F.J. Dyson. Statistical theory of the energy levels of complex systems. V. J. Math. Phys., 4:713-719, 1963.

[33] T. Nagao and P.J. Forrester. Asymptotic correlations at the spectrum edge of random matrices. Nucl. Phys. B, 435:401-420, 1995.

[34] T. Nagao and M. Wadati. Correlation functions of random matrix ensembles related to classical orthogonal polynomials II, III. J. Phys. Soc. Japan, 61:78-88,1910-1918, 1992.

[35] F. Olver. Asymptotics and Special Functions. Academic Press, London, 1974.

[36] E.M. Rains. Correlation functions for symmetrized increasing subsequences. arXiv:math.CO/006097, 2000.

[37] G. Szegö. Orthogonal polynomials. American Mathematical Society, Providence R.I., 4th edition, 1975. 
[38] C.A. Tracy and H. Widom. Fredholm determinants, differential equations and matrix models. Commun. Math. Phys., 163:33-72, 1994.

[39] E.T. Whittaker and G.N. Watson. A course of modern analysis. CUP, Cambridge, 2nd edition, 1965.

[40] H. Widom. On the relation between orthogonal, symplectic and unitary random matrices. J. Stat. Phys., 94:347-364, 1999. 